\title{
Structural and Thermodynamic Properties of the Curium(III) Ion Solvated by Water and Methanol
}

\author{
Morgan P. Kelley, ${ }^{*}, 2$, Ping Yang*,2, Sue B. Clark¹, Aurora E. Clark*,1
}

1. Department of Chemistry, Washington State University, Pullman, WA, 99164

2. Theoretical Division, Los Alamos National Laboratory, Los Alamos, NM, 87544 
Figure S1. Depictions of the tri-capped trigonal prismatic 9 coordinate $\mathrm{Cm}^{3+}$ ion. Numbers represent solvent molecules; 1-3 are equatorial, 4-9 are axial. Panel (a) shows a view perpendicular to the equatorial plane, while panel (b) shows of topdown view of the equatorial plane (axial molecules above the plane are offset for clarity).

(a)

(b)

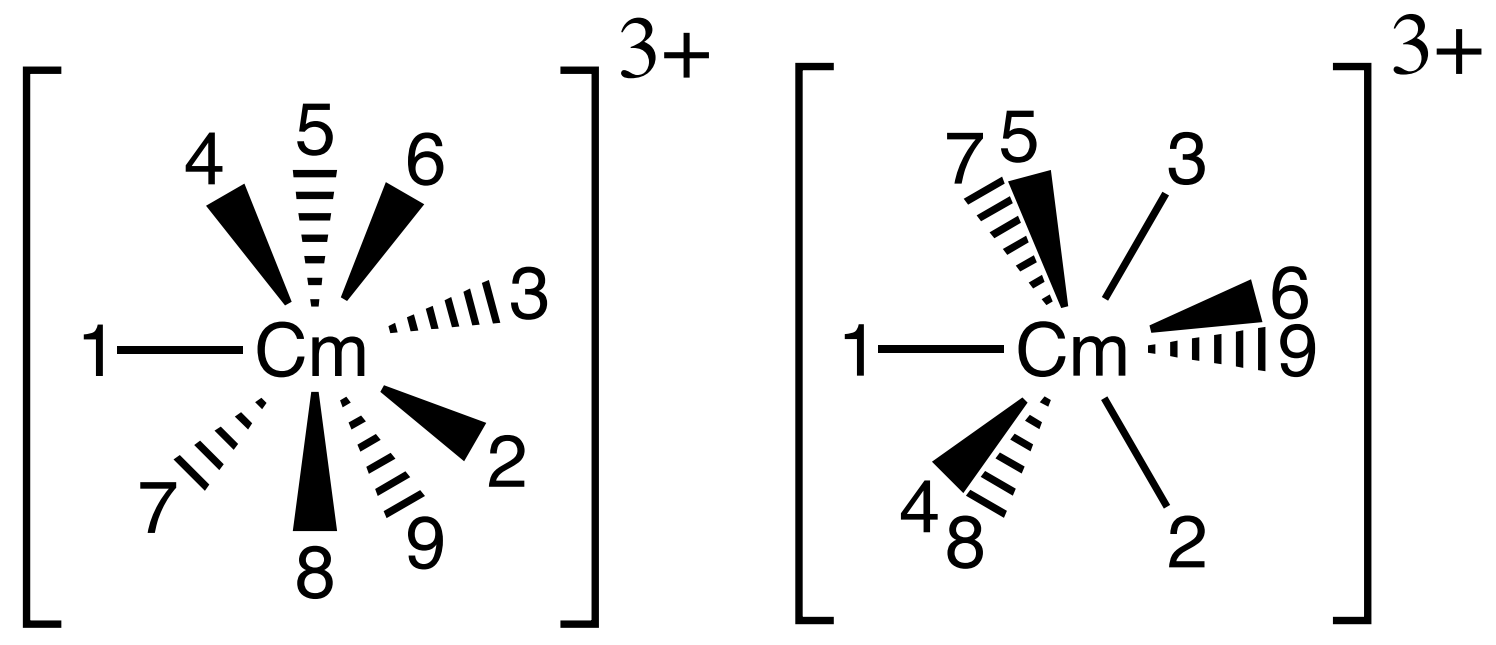

Table S1. Identity of water and methanol molecules in each structure. Numbers correspond to the solvent numbers identified in Supplemental Figure 1.

\begin{tabular}{lcll}
\hline \multicolumn{1}{c}{ Composition } & $\begin{array}{c}\text { Number of } \\
\text { Structures }\end{array}$ & Water Molecules & Methanol Molecules \\
\hline $\mathrm{Cm}^{3+}\left(\mathrm{H}_{\mathbf{2}} \mathbf{O}\right)_{9}$ & 1 & $1,2,3,4,5,6,7,8,9$ & - \\
\hline $\mathrm{Cm}^{3+}\left(\mathrm{H}_{\mathbf{2}} \mathbf{O}\right)_{8}(\mathrm{MeOH})_{1}$ & 2 & $2,3,4,5,6,7,8,9$ & 1 \\
& & $1,2,3,5,6,7,8,9$ & 4 \\
\hline $\mathrm{Cm}^{3+}\left(\mathrm{H}_{\mathbf{2}} \mathrm{O}\right)_{7}(\mathrm{MeOH})_{2}$ & 6 & $2,3,5,6,7,8,9$ & 1,4 \\
& & $2,3,4,6,7,8,9$ & 1,5 \\
& & $3,4,5,6,7,8,9$ & 1,2 \\
& & $1,2,3,6,7,8,9$ & 4,5 \\
& & $1,2,3,5,6,8,9$ & 4,7 \\
& & $1,2,3,5,6,7,8$ & 4,8 \\
\hline
\end{tabular}




\begin{tabular}{|c|c|c|c|}
\hline \multirow[t]{11}{*}{$\mathrm{Cm}^{3+}\left(\mathrm{H}_{2} \mathrm{O}\right)_{6}(\mathrm{MeOH})_{3}$} & 11 & $4,5,6,7,8,9$ & $1,2,3$ \\
\hline & & $3,5,6,7,8,9$ & $1,2,4$ \\
\hline & & $3,4,6,7,8,9$ & $1,2,5$ \\
\hline & & $2,3,6,7,8,9$ & $1,4,5$ \\
\hline & & $2,3,5,7,8,9$ & $1,4,6$ \\
\hline & & $2,3,5,6,8,9$ & $1,4,7$ \\
\hline & & $2,3,5,6,7,9$ & $1,4,8$ \\
\hline & & $2,3,5,6,7,8$ & $1,4,9$ \\
\hline & & $1,2,3,7,8,9$ & $4,5,6$ \\
\hline & & $1,2,3,6,8,9$ & $4,5,7$ \\
\hline & & $1,2,3,6,7,8$ & $4,5,9$ \\
\hline \multirow[t]{17}{*}{$\mathrm{Cm}^{3+}\left(\mathrm{H}_{2} \mathrm{O}\right)_{5}(\mathrm{MeOH})_{4}$} & 17 & $5,6,7,8,9$ & $1,2,3,4$ \\
\hline & & $3,6,7,8,9$ & $1,2,4,5$ \\
\hline & & $3,4,6,8,9$ & $1,2,5,6$ \\
\hline & & $3,4,6,8,9$ & $1,2,5,7$ \\
\hline & & $3,4,6,7,9$ & $1,2,5,8$ \\
\hline & & $3,4,6,7,8$ & $1,2,5,9$ \\
\hline & & $3,5,6,8,9$ & $1,2,4,7$ \\
\hline & & $3,5,6,7,9$ & $1,2,4,8$ \\
\hline & & $2,3,5,8,9$ & $1,4,5,6$ \\
\hline & & $2,3,5,8,9$ & $1,4,6,7$ \\
\hline & & $2,3,5,7,9$ & $1,4,6,8$ \\
\hline & & $2,3,6,8,9$ & $1,4,5,7$ \\
\hline & & $2,3,6,7,9$ & $1,4,5,8$ \\
\hline & & $2,3,6,7,8$ & $1,4,5,9$ \\
\hline & & $1,2,38,9$ & $4,5,6,7$ \\
\hline & & $1,2,3,6,9$ & $4,5,7,8$ \\
\hline & & $1,2,3,6,8$ & $4,5,7,9$ \\
\hline \multirow[t]{17}{*}{$\mathrm{Cm}^{3+}\left(\mathrm{H}_{2} \mathrm{O}\right)_{4}(\mathrm{MeOH})_{5}$} & 17 & $6,7,8,9$ & $1,2,3,4,5$ \\
\hline & & $5,6,8,9$ & $1,2,3,4,7$ \\
\hline & & $5,6,7,9$ & $1,2,3,4,8$ \\
\hline & & $3,7,8,9$ & $1,2,4,5,6$ \\
\hline & & $3,6,8,9$ & $1,2,4,5,7$ \\
\hline & & $3,6,7,9$ & $1,2,4,5,8$ \\
\hline & & $3,6,7,8$ & $1,2,4,5,9$ \\
\hline & & $3,5,8,9$ & $1,2,4,6,7$ \\
\hline & & $3,5,7,9$ & $1,2,4,6,8$ \\
\hline & & $2,3,8,9$ & $1,4,5,6,7$ \\
\hline & & $2,3,7,9$ & $1,4,5,6,8$ \\
\hline & & $2,3,5,9$ & $1,4,6,7,8$ \\
\hline & & $2,3,5,8$ & $1,4,6,7,9$ \\
\hline & & $2,3,6,9$ & $1,4,5,7,8$ \\
\hline & & $2,3,6,8$ & $1,4,5,7,9$ \\
\hline & & $2,3,6,7$ & $1,4,5,8,9$ \\
\hline & & $1,2,3,9$ & $4,5,6,7,8$ \\
\hline
\end{tabular}




\begin{tabular}{|c|c|c|c|}
\hline \multirow[t]{11}{*}{$\mathrm{Cm}^{3+}\left(\mathrm{H}_{2} \mathrm{O}\right)_{3}(\mathrm{MeOH})_{6}$} & 11 & $7,8,9$ & $1,2,3,4,5,6$ \\
\hline & & $6,7,9$ & $1,2,3,4,5,8$ \\
\hline & & $6,7,8$ & $1,2,3,4,5,9$ \\
\hline & & $3,8,9$ & $1,2,4,5,6,7$ \\
\hline & & $3,7,9$ & $1,2,4,5,6,8$ \\
\hline & & $3,6,9$ & $1,2,4,5,7,8$ \\
\hline & & $3,6,8$ & $1,2,4,5,7,9$ \\
\hline & & $3,6,7$ & $1,2,4,5,8,9$ \\
\hline & & $2,3,9$ & $1,4,5,6,7,8$ \\
\hline & & $2,3,8$ & $1,4,5,6,7,9$ \\
\hline & & $1,2,3$ & $4,5,6,7,8,9$ \\
\hline \multirow[t]{6}{*}{$\mathrm{Cm}^{3+}\left(\mathrm{H}_{2} \mathrm{O}\right)_{2}(\mathrm{MeOH})_{7}$} & 6 & 8,9 & $1,2,3,4,5,6,7$ \\
\hline & & 6,9 & $1,2,3,4,5,7,8$ \\
\hline & & 6,8 & $1,2,3,4,5,7,9$ \\
\hline & & 3,9 & $1,2,4,5,6,7,8$ \\
\hline & & 3,7 & $1,2,4,5,6,8,9$ \\
\hline & & 2,3 & $1,4,5,6,7,8,9$ \\
\hline \multirow[t]{2}{*}{$\mathrm{Cm}^{3+}\left(\mathrm{H}_{2} \mathrm{O}\right)_{1}(\mathrm{MeOH})_{8}$} & 2 & 9 & $1,2,3,4,5,6,7,8$ \\
\hline & & 3 & $1,2,4,5,6,7,8,9$ \\
\hline $\mathrm{Cm}^{3+}(\mathrm{MeOH})_{9}$ & 1 & - & $1,2,3,4,5,6,7,8,9$ \\
\hline
\end{tabular}

Table S2. Ion-solvent distances for the $7^{\text {th }} \mathrm{Cm}^{3+}\left(\mathrm{H}_{2} \mathrm{O}\right)_{4}(\mathrm{MeOH})_{5}$ DFT optimized structure using the B3LYP and PBE functionals.

\begin{tabular}{|c|c|c|c|}
\hline \multirow[b]{2}{*}{ Solvent Number } & \multirow[b]{2}{*}{ Solvent Type } & \multicolumn{2}{|c|}{ Ion-Solvent Distance (Å) } \\
\hline & & B3LYP & PBE \\
\hline 1 & Methanol & 2.532 & 2.519 \\
\hline 2 & Methanol & 2.506 & 2.484 \\
\hline 3 & Water & 2.654 & 2.64 \\
\hline 4 & Methanol & 2.612 & 2.619 \\
\hline 5 & Methanol & 2.533 & 2.519 \\
\hline 6 & Water & 2.542 & 2.535 \\
\hline 7 & Water & 2.612 & 2.607 \\
\hline 8 & Water & 2.521 & 2.509 \\
\hline 9 & Methanol & 2.525 & 2.511 \\
\hline
\end{tabular}


Table S3. Average natural charge, ion-solvent distance, and ion-solvent dissociation energy for water.

\begin{tabular}{cccc}
\hline Structure & Natural Charge & $\begin{array}{c}\text { Ion-Solvent } \\
\text { Distance }(\AA)\end{array}$ & $\begin{array}{c}\text { Solvent Dissociation } \\
\text { Energy (kCal/mol) }\end{array}$ \\
\hline$\left[\mathrm{Cm}\left(\mathrm{CH}_{3} \mathrm{OH}\right)_{9}\right]^{3+}$ & - & - & - \\
{$\left[\mathrm{Cm}\left(\mathrm{H}_{2} \mathrm{O}\right)_{1}(\mathrm{CH} 3 \mathrm{OH})_{8}\right]^{3+}$} & 0.103 & $2.67 \pm 0.02$ & $32.5 \pm 3.1$ \\
{$\left[\mathrm{Cm}\left(\mathrm{H}_{2} \mathrm{O}\right)_{2}\left(\mathrm{CH}_{3} \mathrm{OH}\right)_{7}\right]^{3+}$} & $0.121 \pm 0.007$ & $2.56 \pm 0.05$ & $34.0 \pm 0.9$ \\
{$\left[\mathrm{Cm}\left(\mathrm{H}_{2} \mathrm{O}\right)_{3}\left(\mathrm{CH}_{3} \mathrm{OH}\right)_{6}\right]^{3+}$} & $0.121 \pm 0.005$ & $2.56 \pm 0.08$ & $34.4 \pm 1.5$ \\
{$\left[\mathrm{Cm}\left(\mathrm{H}_{2} \mathrm{O}\right)_{4}\left(\mathrm{CH}_{3} \mathrm{OH}\right)_{5}\right]^{3+}$} & $0.123 \pm 0.006$ & $2.53 \pm 0.06$ & $35.0 \pm 1.2$ \\
{$\left[\mathrm{Cm}\left(\mathrm{H}_{2} \mathrm{O}\right)_{5}\left(\mathrm{CH}_{3} \mathrm{OH}\right)_{4}\right]^{3+}$} & $0.121 \pm 0.006$ & $2.55 \pm 0.04$ & $35.5 \pm 1.2$ \\
{$\left[\mathrm{Cm}\left(\mathrm{H}_{2} \mathrm{O}\right)_{6}\left(\mathrm{CH}_{3} \mathrm{OH}\right)_{3}\right]^{3+}$} & $0.129 \pm 0.004$ & $2.53 \pm 0.03$ & $36.1 \pm 0.9$ \\
{$\left[\mathrm{Cm}\left(\mathrm{H}_{2} \mathrm{O}\right)_{7}\left(\mathrm{CH} \mathrm{CH}_{3} \mathrm{OH}\right)_{2}\right]^{3+}$} & $0.129 \pm 0.001$ & $2.53 \pm 0.04$ & $37.0 \pm 1.0$ \\
{$\left[\mathrm{Cm}\left(\mathrm{H}_{2} \mathrm{O}\right)_{8}(\mathrm{CH} 3 \mathrm{OH})_{1}\right]^{3+}$} & $0.134 \pm 0.003$ & $2.52 \pm 0.02$ & $37.5 \pm 0.9$ \\
{$\left[\mathrm{Cm}\left(\mathrm{H}_{2} \mathrm{O}\right)_{9}\right]^{3+}$} & $0.137 \pm 0.003$ & $2.51 \pm 0.02$ & $38.4 \pm 0.8$ \\
\hline
\end{tabular}

Table S4. Average natural charge, ion-solvent distance, and ion-solvent dissociation energy for methanol.

\begin{tabular}{cccc}
\hline Structure & Natural Charge & $\begin{array}{c}\text { Ion-Solvent } \\
\text { Distance }(\AA)\end{array}$ & $\begin{array}{c}\text { Solvent Dissociation } \\
\text { Energy (kCal/mol) }\end{array}$ \\
\hline$\left[\mathrm{Cm}\left(\mathrm{CH}_{3} \mathrm{OH}\right)_{9}\right]^{3+}$ & $0.126 \pm 0.007$ & $2.52 \pm 0.08$ & $42.5 \pm 1.7$ \\
{$\left[\mathrm{Cm}\left(\mathrm{H}_{2} \mathrm{O}\right)_{1}\left(\mathrm{CH} \mathrm{CH}_{3} \mathrm{OH}\right)_{8}\right]^{3+}$} & $0.131 \pm 0.008$ & $2.51 \pm 0.11$ & $43.2 \pm 1.4$ \\
{$\left[\mathrm{Cm}\left(\mathrm{H}_{2} \mathrm{O}\right)_{2}\left(\mathrm{CH}_{3} \mathrm{OH}\right)_{7}\right]^{3+}$} & $0.135 \pm 0.004$ & $2.50 \pm 0.03$ & $43.4 \pm 0.9$ \\
{$\left[\mathrm{Cm}\left(\mathrm{H}_{2} \mathrm{O}\right)_{3}\left(\mathrm{CH}_{3} \mathrm{OH}\right)_{6}\right]^{3+}$} & $0.139 \pm 0.004$ & $2.47 \pm 0.08$ & $44.3 \pm 1.1$ \\
{$\left[\mathrm{Cm}\left(\mathrm{H}_{2} \mathrm{O}\right)_{4}\left(\mathrm{CH}_{3} \mathrm{OH}\right)_{5}\right]^{3+}$} & $0.145 \pm 0.005$ & $2.46 \pm 0.05$ & $44.9 \pm 1.1$ \\
{$\left[\mathrm{Cm}\left(\mathrm{H}_{2} \mathrm{O}\right)_{5}\left(\mathrm{CH}_{3} \mathrm{OH}\right)_{4}\right]^{3+}$} & $0.151 \pm 0.002$ & $2.46 \pm 0.05$ & $45.6 \pm 0.9$ \\
{$\left[\mathrm{Cm}\left(\mathrm{H}_{2} \mathrm{O}\right)_{6}\left(\mathrm{CH}_{3} \mathrm{OH}\right)_{3}\right]^{3+}$} & $0.151 \pm 0.004$ & $2.47 \pm 0.04$ & $46.1 \pm 0.9$ \\
{$\left[\mathrm{Cm}\left(\mathrm{H}_{2} \mathrm{O}\right)_{7}\left(\mathrm{CH}_{3} \mathrm{OH}\right)_{2}\right]^{3+}$} & $0.159 \pm 0.000$ & $2.45 \pm 0.02$ & $47.0 \pm 0.6$ \\
{$\left[\mathrm{Cm}\left(\mathrm{H}_{2} \mathrm{O}\right)_{8}\left(\mathrm{CH} \mathrm{CH}_{3} \mathrm{OH}\right)_{1}\right]^{3+}$} & 0.160 & $2.44 \pm 0.02$ & $40.4 \pm 1.1$ \\
{$\left[\mathrm{Cm}\left(\mathrm{H}_{2} \mathrm{O}\right)_{9}\right]^{3+}$} & - & - & - \\
\hline
\end{tabular}


Cartesian coordinates of each DFT optimized structure follow, starting with the pure methanol system and ending with pure water. Coordinates are in angstroms.

\begin{tabular}{|c|c|c|c|}
\hline $\mathrm{Cm}$ & 0.00145 & 0.00781 & 0.00567 \\
\hline 0 & -2.02060 & 0.51482 & -1.65500 \\
\hline C & -3.25653 & 1.29705 & -1.76749 \\
\hline $\mathrm{H}$ & -2.06990 & -0.22541 & -2.27589 \\
\hline 0 & 0.40036 & -2.29242 & 0.93919 \\
\hline C & 1.49679 & -3.24192 & 1.17193 \\
\hline $\mathrm{H}$ & -0.42635 & -2.71645 & 1.20555 \\
\hline O & 1.71732 & 1.58501 & 1.05686 \\
\hline $\mathrm{C}$ & 2.63130 & 2.70910 & 0.80185 \\
\hline $\mathrm{H}$ & 1.66066 & 1.44572 & 2.01326 \\
\hline O & -2.12781 & -1.01514 & 1.02572 \\
\hline $\mathrm{C}$ & -3.41163 & -1.60771 & 0.62108 \\
\hline $\mathrm{H}$ & -2.10685 & -0.96414 & 1.99212 \\
\hline O & -0.03358 & 0.16391 & 2.68766 \\
\hline C & 0.43941 & -0.58869 & 3.86232 \\
\hline $\mathrm{H}$ & -0.52694 & 0.93662 & 2.99882 \\
\hline 0 & -1.35519 & 1.98305 & 0.87650 \\
\hline C & -1.16437 & 3.44194 & 0.89583 \\
\hline $\mathrm{H}$ & -2.29856 & 1.80744 & 1.00008 \\
\hline O & -0.20580 & -1.55435 & -2.02857 \\
\hline $\mathrm{C}$ & -0.53955 & -2.97184 & -2.23402 \\
\hline $\mathrm{H}$ & 0.25881 & -1.23970 & -2.81747 \\
\hline 0 & 2.38350 & -0.58801 & -0.52972 \\
\hline C & 3.15559 & -1.47306 & -1.40796 \\
\hline $\mathrm{H}$ & 2.99630 & -0.00829 & -0.05740 \\
\hline O & 0.53897 & 1.41329 & -1.98011 \\
\hline C & 1.63866 & 2.00585 & -2.75038 \\
\hline $\mathrm{H}$ & -0.30343 & 1.66404 & -2.38853 \\
\hline $\mathrm{H}$ & -3.06589 & 2.27097 & -1.31007 \\
\hline $\mathrm{H}$ & -4.07956 & 0.78627 & -1.25572 \\
\hline $\mathrm{H}$ & -3.50612 & 1.44151 & -2.82373 \\
\hline $\mathrm{H}$ & 2.42459 & -2.66886 & 1.15225 \\
\hline $\mathrm{H}$ & 1.49922 & -4.00724 & 0.38917 \\
\hline $\mathrm{H}$ & 1.37058 & -3.71005 & 2.15317 \\
\hline $\mathrm{H}$ & -3.38669 & -1.70652 & -0.46591 \\
\hline $\mathrm{H}$ & -4.23613 & -0.95432 & 0.92435 \\
\hline $\mathrm{H}$ & -3.52545 & -2.59415 & 1.08218 \\
\hline $\mathrm{H}$ & 1.08312 & -1.38520 & 3.48480 \\
\hline $\mathrm{H}$ & -0.41218 & -1.01239 & 4.40560 \\
\hline $\mathrm{H}$ & 1.01088 & 0.07237 & 4.52240 \\
\hline $\mathrm{H}$ & -0.10109 & 3.62035 & 0.73015 \\
\hline $\mathrm{H}$ & -1.46913 & 3.83922 & 1.86932 \\
\hline $\mathrm{H}$ & -1.75104 & 3.90772 & 0.09693 \\
\hline $\mathrm{H}$ & -1.16465 & -3.27569 & -1.39158 \\
\hline $\mathrm{H}$ & 0.37370 & -3.57495 & -2.26423 \\
\hline $\mathrm{H}$ & -1.09703 & -3.08470 & -3.16925 \\
\hline $\mathrm{H}$ & 2.47928 & -2.26186 & -1.74250 \\
\hline $\mathrm{H}$ & 3.98674 & -1.91469 & -0.84909 \\
\hline
\end{tabular}




$\begin{array}{rrrr}\mathrm{H} & 3.53768 & -0.90822 & -2.26499 \\ \mathrm{H} & 2.56834 & 1.61835 & -2.32940 \\ \mathrm{H} & 1.60403 & 3.09688 & -2.66522 \\ \mathrm{H} & 1.55365 & 1.70784 & -3.80029 \\ \mathrm{H} & 2.57219 & 2.93483 & -0.26412 \\ \mathrm{H} & 3.65324 & 2.42613 & 1.07651 \\ \mathrm{H} & 2.31756 & 3.58058 & 1.38547\end{array}$

52

$\begin{array}{lrrr}\mathrm{Cm} & 0.06482 & 0.03540 & -0.08745 \\ \mathrm{O} & -1.76764 & 1.40855 & -1.21761 \\ \mathrm{C} & -2.36760 & 2.74681 & -1.26826 \\ \mathrm{H} & -2.32505 & 0.79701 & -1.71966 \\ \mathrm{O} & -0.64472 & -2.04766 & 1.10327 \\ \mathrm{C} & -0.28291 & -3.46446 & 1.24261 \\ \mathrm{H} & -1.41881 & -1.87570 & 1.65595 \\ \mathrm{O} & 2.58181 & 0.66613 & -0.10127 \\ \mathrm{C} & 3.63434 & 1.25927 & -0.94120 \\ \mathrm{H} & 2.92963 & 0.56502 & 0.79580 \\ \mathrm{O} & -1.81076 & 0.43038 & 1.70237 \\ \mathrm{C} & -3.26828 & 0.64613 & 1.72697 \\ \mathrm{H} & -1.49424 & 0.45757 & 2.61664 \\ \mathrm{O} & 1.16869 & 0.09499 & 2.25196 \\ \mathrm{C} & 1.62310 & -0.86850 & 3.26947 \\ \mathrm{H} & 1.24084 & 0.98803 & 2.61852 \\ \mathrm{O} & 0.21190 & 2.41634 & 0.80752 \\ \mathrm{C} & 1.04500 & 3.61489 & 0.61592 \\ \mathrm{H} & -0.61201 & 2.69335 & 1.23272 \\ \mathrm{O} & -1.44935 & -1.26259 & -1.74855 \\ \mathrm{C} & -2.50384 & -2.28440 & -1.66812 \\ \mathrm{H} & -1.11963 & -1.24253 & -2.65865 \\ \mathrm{O} & 1.57214 & -1.75855 & -0.98726 \\ \mathrm{C} & 1.52660 & -3.00427 & -1.76402 \\ \mathrm{H} & 2.49791 & -1.50875 & -0.85875 \\ \mathrm{O} & 0.65149 & 0.69541 & -2.49918 \\ \mathrm{H} & 1.39117 & 0.42746 & -3.06461 \\ \mathrm{H} & 0.20104 & 1.41392 & -2.96822 \\ \mathrm{H} & -1.59568 & 3.45831 & -0.96773 \\ \mathrm{H} & -3.22645 & 2.80180 & -0.59070 \\ \mathrm{H} & -2.68472 & 2.96768 & -2.29260 \\ \mathrm{H} & 0.74081 & -3.57068 & 0.88124 \\ \mathrm{H} & -0.96673 & -4.08232 & 0.65196 \\ \mathrm{H} & -0.33460 & -3.74999 & 2.29778 \\ \mathrm{H} & 3.14713 & 1.67120 & -1.82777 \\ \mathrm{H} & 4.36534 & 0.49367 & -1.22337 \\ \mathrm{H} & 4.13472 & 2.06375 & -0.39255 \\ \mathrm{H} & -3.62254 & 0.52141 & 0.70194 \\ \mathrm{H} & -3.49002 & 1.65631 & 2.08656 \\ \mathrm{H} & -3.74123 & -0.09580 & 2.37853 \\ \mathrm{H} & 1.70179 & -1.83751 & 2.77325 \\ \mathrm{H} & 0.89954 & -0.91659 & 4.08999 \\ \mathrm{H} & 2.60339 & -0.56461 & 3.65053 \\ & & & \end{array}$




$\begin{array}{rrrr}\mathrm{H} & 1.97272 & 3.28054 & 0.15031 \\ \mathrm{H} & 1.25264 & 4.07507 & 1.58739 \\ \mathrm{H} & 0.53167 & 4.32735 & -0.03842 \\ \mathrm{H} & -2.93319 & -2.21878 & -0.66613 \\ \mathrm{H} & -2.07897 & -3.27978 & -1.83386 \\ \mathrm{H} & -3.27444 & -2.07562 & -2.41698 \\ \mathrm{H} & 0.48329 & -3.32297 & -1.79391 \\ \mathrm{H} & 2.13638 & -3.76837 & -1.27135 \\ \mathrm{H} & 1.89867 & -2.82328 & -2.77798\end{array}$

52

$\begin{array}{lrrr}\mathrm{Cm} & -0.04084 & -0.00258 & 0.13790 \\ \mathrm{O} & -0.68533 & -1.47940 & -1.80568 \\ \mathrm{C} & -0.63337 & -2.85826 & -2.30804 \\ \mathrm{H} & -1.12015 & -0.92271 & -2.46810 \\ \mathrm{O} & 1.52905 & 1.57223 & -0.95579 \\ \mathrm{C} & 1.80555 & 2.99271 & -1.20421 \\ \mathrm{H} & 2.28220 & 1.04828 & -1.26558 \\ \mathrm{O} & -0.85944 & -0.13661 & 2.77084 \\ \mathrm{H} & -1.72391 & -0.40083 & 3.12012 \\ \mathrm{H} & -0.25717 & -0.19873 & 3.52720 \\ \mathrm{O} & 2.27250 & -1.08960 & -0.85737 \\ \mathrm{C} & 2.74540 & -1.69356 & -2.11678 \\ \mathrm{H} & 3.01611 & -1.08158 & -0.23804 \\ \mathrm{O} & 1.86754 & 0.18599 & 1.81138 \\ \mathrm{C} & 2.68295 & 1.31282 & 2.29573 \\ \mathrm{H} & 2.10766 & -0.60360 & 2.31680 \\ \mathrm{O} & 0.39928 & -2.32413 & 0.96832 \\ \mathrm{C} & -0.27692 & -3.36233 & 1.76088 \\ \mathrm{H} & 1.13639 & -2.73243 & 0.49219 \\ \mathrm{O} & -1.37320 & 1.19183 & -1.75731 \\ \mathrm{C} & -1.09658 & 1.98144 & -2.96793 \\ \mathrm{H} & -2.31605 & 1.27383 & -1.55615 \\ \mathrm{O} & -0.85073 & 2.08698 & 1.18369 \\ \mathrm{C} & -1.29789 & 3.45188 & 0.87756 \\ \mathrm{H} & -0.94349 & 1.93446 & 2.13641 \\ \mathrm{O} & -2.49984 & -0.59632 & 0.29972 \\ \mathrm{C} & -3.69882 & -0.20710 & 1.05496 \\ \mathrm{H} & -2.72823 & -1.35459 & -0.25661 \\ \mathrm{H} & -0.10661 & -3.45357 & -1.55991 \\ \mathrm{H} & -0.09415 & -2.88690 & -3.26001 \\ \mathrm{H} & -1.65053 & -3.24139 & -2.44096 \\ \mathrm{H} & 0.95008 & 3.55682 & -0.83104 \\ \mathrm{H} & 1.92973 & 3.16048 & -2.27853 \\ \mathrm{H} & 2.71308 & 3.28845 & -0.66838 \\ \mathrm{H} & 1.99175 & -1.47004 & -2.87451 \\ \mathrm{H} & 2.85902 & -2.77587 & -1.99576 \\ \mathrm{H} & 3.70324 & -1.24776 & -2.40397 \\ \mathrm{H} & 2.34117 & 2.19846 & 1.75672 \\ \mathrm{H} & 3.74030 & 1.12262 & 2.08481 \\ \mathrm{H} & 2.53019 & 1.44588 & 3.37163 \\ \mathrm{H} & -1.07968 & -2.86655 & 2.30909\end{array}$




$\begin{array}{rrrr}\mathrm{H} & 0.43521 & -3.80970 & 2.46160 \\ \mathrm{H} & -0.69016 & -4.12808 & 1.09596 \\ \mathrm{H} & -0.07531 & 1.74352 & -3.27192 \\ \mathrm{H} & -1.19082 & 3.05062 & -2.75168 \\ \mathrm{H} & -1.79782 & 1.69647 & -3.75885 \\ \mathrm{H} & -1.31074 & 3.54681 & -0.20966 \\ \mathrm{H} & -0.60272 & 4.17396 & 1.31774 \\ \mathrm{H} & -2.30602 & 3.60494 & 1.27544 \\ \mathrm{H} & -3.45853 & 0.72353 & 1.57512 \\ \mathrm{H} & -3.96570 & -0.99601 & 1.76726 \\ \mathrm{H} & -4.53105 & -0.03540 & 0.36448\end{array}$

49

$\begin{array}{lrrr}\mathrm{Cm} & 0.07754 & 0.03932 & -0.16010 \\ \mathrm{O} & -2.34418 & 0.69603 & -0.52926 \\ \mathrm{C} & -3.36197 & 1.68319 & -0.14655 \\ \mathrm{H} & -2.76735 & 0.00456 & -1.05802 \\ \mathrm{O} & 0.50482 & -2.31793 & 0.57816 \\ \mathrm{C} & 1.28894 & -3.50971 & 0.22446 \\ \mathrm{H} & -0.06502 & -2.54084 & 1.32661 \\ \mathrm{O} & 2.12663 & 1.50686 & -0.54236 \\ \mathrm{C} & 2.70259 & 2.50693 & -1.45393 \\ \mathrm{H} & 2.73469 & 1.38623 & 0.20040 \\ \mathrm{O} & -1.20562 & -0.60416 & 2.00686 \\ \mathrm{C} & -2.57771 & -0.98679 & 2.38679 \\ \mathrm{H} & -0.67464 & -0.56527 & 2.81515 \\ \mathrm{O} & 1.70015 & 0.15752 & 1.86374 \\ \mathrm{C} & 2.69888 & -0.70701 & 2.51862 \\ \mathrm{H} & 1.59450 & 0.95923 & 2.39602 \\ \mathrm{O} & -0.33293 & 2.14219 & 1.19139 \\ \mathrm{C} & -0.00949 & 3.57632 & 1.08803 \\ \mathrm{H} & -1.03609 & 2.04422 & 1.84903 \\ \mathrm{O} & -1.24259 & -1.49824 & -1.79634 \\ \mathrm{C} & -1.81991 & -2.85221 & -1.79978 \\ \mathrm{H} & -1.24736 & -1.16750 & -2.70636 \\ \mathrm{O} & 1.72175 & -0.96589 & -1.84536 \\ \mathrm{H} & 1.63671 & -1.76851 & -2.38129 \\ \mathrm{H} & 2.62357 & -0.64307 & -1.98866 \\ \mathrm{O} & -0.26570 & 1.20995 & -2.42202 \\ \mathrm{H} & 0.34919 & 1.29018 & -3.16684 \\ \mathrm{H} & -1.01127 & 1.79470 & -2.62609 \\ \mathrm{H} & -2.83337 & 2.56518 & 0.22063 \\ \mathrm{H} & -4.01075 & 1.27103 & 0.63360 \\ \mathrm{H} & -3.95639 & 1.95507 & -1.02484 \\ \mathrm{H} & 2.15256 & -3.17010 & -0.35069 \\ \mathrm{H} & 0.67529 & -4.19722 & -0.36675 \\ \mathrm{H} & 1.63130 & -4.00381 & 1.13912 \\ \mathrm{H} & 1.89621 & 2.85803 & -2.10143 \\ \mathrm{H} & 3.50567 & 2.05639 & -2.04738 \\ \mathrm{H} & 3.09672 & 3.34789 & -0.87415 \\ \mathrm{H} & -3.13087 & -1.13989 & 1.45817 \\ \mathrm{H} & -3.03708 & -0.18824 & 2.97839 \\ & & & \end{array}$




$\begin{array}{rrrr}\mathrm{H} & -2.55390 & -1.91620 & 2.96479 \\ \mathrm{H} & 2.91816 & -1.51770 & 1.82153 \\ \mathrm{H} & 2.29258 & -1.10611 & 3.45398 \\ \mathrm{H} & 3.60770 & -0.13063 & 2.71952 \\ \mathrm{H} & 0.78773 & 3.66431 & 0.34891 \\ \mathrm{H} & 0.33245 & 3.94424 & 2.06074 \\ \mathrm{H} & -0.89246 & 4.13460 & 0.75973 \\ \mathrm{H} & -1.88831 & -3.16949 & -0.75705 \\ \mathrm{H} & -1.17532 & -3.53385 & -2.36435 \\ \mathrm{H} & -2.81892 & -2.82473 & -2.24669\end{array}$

49

$\begin{array}{lrrr}\mathrm{Cm} & 0.13371 & 0.06923 & -0.02726 \\ \mathrm{O} & -0.33204 & 2.35289 & -1.09285 \\ \mathrm{C} & 0.00601 & 3.77693 & -0.96043 \\ \mathrm{H} & -1.08991 & 2.27288 & -1.68864 \\ \mathrm{O} & -1.73118 & -1.40462 & 0.74492 \\ \mathrm{C} & -2.18771 & -2.80071 & 0.71749 \\ \mathrm{H} & -2.38324 & -0.87959 & 1.22872 \\ \mathrm{O} & 2.62823 & -0.60123 & 0.31124 \\ \mathrm{C} & 3.94887 & -0.50176 & -0.33413 \\ \mathrm{H} & 2.74842 & -1.00405 & 1.18297 \\ \mathrm{O} & -1.54612 & 1.26055 & 1.53860 \\ \mathrm{C} & -2.60212 & 2.28069 & 1.41737 \\ \mathrm{H} & -1.39856 & 1.10226 & 2.48199 \\ \mathrm{O} & 0.69477 & -0.65278 & 2.37662 \\ \mathrm{C} & 0.40734 & -1.80104 & 3.25470 \\ \mathrm{H} & 1.13630 & 0.03064 & 2.90123 \\ \mathrm{O} & 1.47636 & 1.87419 & 1.27476 \\ \mathrm{H} & 2.44317 & 1.94138 & 1.26711 \\ \mathrm{H} & 1.15877 & 2.72525 & 1.61279 \\ \mathrm{O} & -1.53094 & -0.01919 & -1.95978 \\ \mathrm{C} & -2.96057 & -0.30923 & -2.15479 \\ \mathrm{H} & -1.09453 & -0.05596 & -2.82349 \\ \mathrm{O} & 0.66746 & -2.17805 & -0.98907 \\ \mathrm{C} & 0.09939 & -3.14940 & -1.93576 \\ \mathrm{H} & 1.55957 & -2.46818 & -0.75190 \\ \mathrm{O} & 1.32985 & 0.43301 & -2.28993 \\ \mathrm{H} & 1.88032 & -0.16939 & -2.81234 \\ \mathrm{H} & 1.43362 & 1.30921 & -2.69100 \\ \mathrm{H} & 1.05241 & 3.83555 & -0.65129 \\ \mathrm{H} & -0.64694 & 4.25147 & -0.21950 \\ \mathrm{H} & -0.11075 & 4.27098 & -1.93021 \\ \mathrm{H} & -1.32109 & -3.41764 & 0.47573 \\ \mathrm{H} & -2.96976 & -2.92073 & -0.03904 \\ \mathrm{H} & -2.57326 & -3.07533 & 1.70415 \\ \mathrm{H} & 3.83587 & 0.16397 & -1.19249 \\ \mathrm{H} & 4.28420 & -1.49339 & -0.65742 \\ \mathrm{H} & 4.67186 & -0.07595 & 0.36959 \\ \mathrm{H} & -2.82107 & 2.38542 & 0.35276 \\ \mathrm{H} & -2.25175 & 3.23200 & 1.83058 \\ \mathrm{H} & -3.49813 & 1.94615 & 1.94993\end{array}$




$\begin{array}{rrrr}\mathrm{H} & 0.09903 & -2.62410 & 2.60767 \\ \mathrm{H} & -0.39298 & -1.54411 & 3.95634 \\ \mathrm{H} & 1.31408 & -2.07738 & 3.80220 \\ \mathrm{H} & -3.44773 & -0.16907 & -1.18779 \\ \mathrm{H} & -3.09043 & -1.34005 & -2.49980 \\ \mathrm{H} & -3.37771 & 0.38908 & -2.88714 \\ \mathrm{H} & -0.92785 & -2.83705 & -2.13187 \\ \mathrm{H} & 0.10988 & -4.14869 & -1.48916 \\ \mathrm{H} & 0.68140 & -3.14594 & -2.86327\end{array}$

$\begin{array}{lrrr}\mathrm{Cm} & 0.07734 & 0.07558 & 0.03000 \\ \mathrm{O} & -2.47469 & 0.55976 & -0.00560 \\ \mathrm{C} & -3.56982 & 0.96647 & 0.88810 \\ \mathrm{H} & -2.86265 & 0.15474 & -0.79394 \\ \mathrm{O} & 0.95671 & -2.16183 & -0.71658 \\ \mathrm{C} & 1.95111 & -2.78502 & -1.59968 \\ \mathrm{H} & 0.43080 & -2.86176 & -0.30658 \\ \mathrm{O} & 1.72119 & 1.82459 & 0.91409 \\ \mathrm{C} & 1.91739 & 3.28317 & 0.97543 \\ \mathrm{H} & 2.42554 & 1.40274 & 1.42604 \\ \mathrm{O} & -1.06673 & -1.92001 & 1.19904 \\ \mathrm{C} & -2.35679 & -2.62865 & 1.15250 \\ \mathrm{H} & -0.57454 & -2.25063 & 1.96408 \\ \mathrm{O} & 1.64488 & -0.81903 & 1.88966 \\ \mathrm{C} & 2.79887 & -1.73035 & 1.99513 \\ \mathrm{H} & 1.40319 & -0.53255 & 2.78222 \\ \mathrm{O} & -0.62300 & 0.92685 & 2.37040 \\ \mathrm{H} & -0.30377 & 1.75340 & 2.76330 \\ \mathrm{H} & -1.38175 & 0.65773 & 2.91008 \\ \mathrm{O} & -1.00553 & -0.42184 & -2.22738 \\ \mathrm{C} & -1.31635 & -1.58339 & -3.07708 \\ \mathrm{H} & -1.11426 & 0.37832 & -2.76108 \\ \mathrm{O} & 1.94801 & 0.49316 & -1.62411 \\ \mathrm{H} & 2.07566 & 0.14683 & -2.52003 \\ \mathrm{H} & 2.70340 & 1.07411 & -1.45086 \\ \mathrm{O} & -0.57742 & 2.24195 & -1.10758 \\ \mathrm{C} & -0.05215 & 3.30802 & -1.97371 \\ \mathrm{H} & -1.50740 & 2.43145 & -0.91584 \\ \mathrm{H} & -3.17654 & 1.74276 & 1.54916 \\ \mathrm{H} & -3.92590 & 0.10553 & 1.46429 \\ \mathrm{H} & -4.38943 & 1.38264 & 0.29375 \\ \mathrm{H} & 2.77304 & -2.07548 & -1.71791 \\ \mathrm{H} & 1.49655 & -3.01773 & -2.56842 \\ \mathrm{H} & 2.32853 & -3.70073 & -1.13383 \\ \mathrm{H} & 0.98206 & 3.74335 & 0.65289 \\ \mathrm{H} & 2.73954 & 3.57810 & 0.31487 \\ \mathrm{H} & 2.14285 & 3.57772 & 2.00558 \\ \mathrm{H} & -2.86002 & -2.31245 & 0.23631 \\ \mathrm{H} & -2.95841 & -2.36782 & 2.02913 \\ \mathrm{H} & -2.18158 & -3.70889 & 1.12476 \\ \mathrm{H} & 3.17144 & -1.88572 & 0.98130\end{array}$




$\begin{array}{rrrr}\mathrm{H} & 2.48477 & -2.68139 & 2.43787 \\ \mathrm{H} & 3.57583 & -1.26715 & 2.61176 \\ \mathrm{H} & -1.25691 & -2.46731 & -2.43921 \\ \mathrm{H} & -0.59099 & -1.65273 & -3.89417 \\ \mathrm{H} & -2.32901 & -1.48071 & -3.47984 \\ \mathrm{H} & 1.00183 & 3.08619 & -2.14947 \\ \mathrm{H} & -0.15887 & 4.27692 & -1.47569 \\ \mathrm{H} & -0.59742 & 3.31377 & -2.92352\end{array}$

$\begin{array}{lrrr}\mathrm{Cm} & 0.15762 & 0.02795 & -0.12956 \\ \mathrm{O} & -0.89012 & 2.12012 & -1.13701 \\ \mathrm{C} & -0.84446 & 3.58577 & -1.02393 \\ \mathrm{H} & -1.65798 & 1.87990 & -1.67408 \\ \mathrm{O} & -1.14563 & -1.97472 & 0.61681 \\ \mathrm{H} & -0.80841 & -2.74419 & 0.13632 \\ \mathrm{C} & -2.19728 & -2.41732 & 1.53873 \\ \mathrm{O} & 2.70758 & -0.13360 & -0.18917 \\ \mathrm{H} & 3.30218 & 0.08893 & -0.92047 \\ \mathrm{H} & 3.27757 & -0.43563 & 0.53313 \\ \mathrm{O} & -1.49446 & 0.85987 & 1.59712 \\ \mathrm{C} & -2.78916 & 1.56003 & 1.63367 \\ \mathrm{H} & -1.33309 & 0.48776 & 2.47624 \\ \mathrm{O} & 1.06033 & -0.61679 & 2.15236 \\ \mathrm{C} & 1.23090 & -1.81259 & 2.99334 \\ \mathrm{H} & 1.47202 & 0.13639 & 2.60032 \\ \mathrm{O} & 1.10831 & 2.04890 & 1.12414 \\ \mathrm{C} & 2.31260 & 2.89513 & 1.17044 \\ \mathrm{H} & 0.40523 & 2.50508 & 1.60829 \\ \mathrm{O} & -1.59368 & -0.45141 & -1.97054 \\ \mathrm{C} & -2.93787 & -1.05461 & -2.02028 \\ \mathrm{H} & -1.30051 & -0.29321 & -2.87949 \\ \mathrm{O} & 0.80449 & -2.21909 & -1.30878 \\ \mathrm{H} & 0.30825 & -2.43280 & -2.11138 \\ \mathrm{C} & 1.92275 & -3.17355 & -1.20687 \\ \mathrm{O} & 1.12080 & 0.61550 & -2.49638 \\ \mathrm{H} & 1.68301 & 0.12846 & -3.11804 \\ \mathrm{H} & 1.01999 & 1.50216 & -2.87526 \\ \mathrm{H} & 0.16634 & 3.85148 & -0.70939 \\ \mathrm{H} & -1.58098 & 3.92966 & -0.28962 \\ \mathrm{H} & -1.05295 & 4.03221 & -2.00134 \\ \mathrm{H} & -2.98169 & 1.92171 & 0.62227 \\ \mathrm{H} & -2.73222 & 2.40053 & 2.33276 \\ \mathrm{H} & -3.57929 & 0.86675 & 1.93969 \\ \mathrm{H} & 0.83192 & -2.65584 & 2.42679 \\ \mathrm{H} & 0.67838 & -1.68804 & 3.93034 \\ \mathrm{H} & 2.29429 & -1.96945 & 3.20114 \\ \mathrm{H} & 3.08475 & 2.37784 & 0.59897 \\ \mathrm{H} & 2.63134 & 3.02275 & 2.21029 \\ \mathrm{H} & -2.3018313 & -1.87050 & 0.71943 \\ \mathrm{H} & & -2.06862 & -0.99423 \\ \mathrm{H} & & & -2.42891\end{array}$




$\begin{array}{rrrr}\mathrm{H} & -3.59359 & -0.43612 & -2.64134 \\ \mathrm{H} & 2.35635 & -3.04896 & -0.21217 \\ \mathrm{H} & 2.67059 & -2.96093 & -1.97777 \\ \mathrm{H} & 1.54319 & -4.19422 & -1.31981 \\ \mathrm{H} & -2.72196 & -1.52434 & 1.88337 \\ \mathrm{H} & -1.75279 & -2.94918 & 2.38633 \\ \mathrm{H} & -2.89676 & -3.07136 & 1.00851\end{array}$

49

\begin{tabular}{|c|c|c|c|}
\hline $\mathrm{Cm}$ & -0.06101 & 0.01693 & 0.00412 \\
\hline 0 & -0.15060 & -2.14269 & -1.39539 \\
\hline $\mathrm{C}$ & -0.02177 & -3.59927 & -1.23810 \\
\hline $\mathrm{H}$ & -0.12187 & -1.93573 & -2.34074 \\
\hline O & 1.40049 & 1.86086 & -0.96142 \\
\hline $\mathrm{H}$ & 1.00339 & 2.73351 & -0.83673 \\
\hline $\mathrm{C}$ & 2.75187 & 2.05335 & -1.50931 \\
\hline 0 & -1.59598 & 0.56655 & 2.11210 \\
\hline $\mathrm{H}$ & -2.51261 & 0.29151 & 2.26616 \\
\hline $\mathrm{H}$ & -1.26092 & 0.83525 & 2.98066 \\
\hline 0 & 2.31649 & -0.88602 & -0.10871 \\
\hline $\mathrm{C}$ & 3.17251 & -1.72585 & -0.96316 \\
\hline $\mathrm{H}$ & 2.88835 & -0.41889 & 0.51681 \\
\hline 0 & 1.29759 & 0.87583 & 1.99669 \\
\hline $\mathrm{C}$ & 1.90564 & 2.14010 & 2.44645 \\
\hline $\mathrm{H}$ & 1.39430 & 0.22584 & 2.70763 \\
\hline 0 & 0.24115 & -1.86289 & 1.66058 \\
\hline $\mathrm{C}$ & -0.54737 & -2.63899 & 2.63096 \\
\hline $\mathrm{H}$ & 1.08271 & -2.32009 & 1.51908 \\
\hline 0 & -0.50966 & 0.35456 & -2.54445 \\
\hline $\mathrm{H}$ & -1.36006 & 0.27409 & -3.00340 \\
\hline 0 & -1.27323 & 2.23621 & -0.06448 \\
\hline $\mathrm{C}$ & -1.73445 & 3.26830 & -1.00555 \\
\hline $\mathrm{H}$ & -1.68130 & 2.40572 & 0.79716 \\
\hline 0 & -2.40182 & -0.78061 & -0.41605 \\
\hline $\mathrm{C}$ & -3.79788 & -0.35284 & -0.25834 \\
\hline $\mathrm{H}$ & -2.39313 & -1.67328 & -0.79031 \\
\hline $\mathrm{H}$ & -0.25212 & -3.82804 & -0.19582 \\
\hline $\mathrm{H}$ & 0.99679 & -3.91693 & -1.48363 \\
\hline $\mathrm{H}$ & -0.74033 & -4.10221 & -1.89337 \\
\hline $\mathrm{H}$ & 2.57282 & -2.02057 & -1.82601 \\
\hline $\mathrm{H}$ & 3.50114 & -2.60768 & -0.40350 \\
\hline $\mathrm{H}$ & 4.04114 & -1.14886 & -1.29523 \\
\hline $\mathrm{H}$ & 1.75564 & 2.86546 & 1.64463 \\
\hline $\mathrm{H}$ & 2.97517 & 1.99183 & 2.62786 \\
\hline $\mathrm{H}$ & 1.41172 & 2.48556 & 3.36051 \\
\hline $\mathrm{H}$ & -1.51018 & -2.13523 & 2.73185 \\
\hline $\mathrm{H}$ & -0.02761 & -2.65979 & 3.59438 \\
\hline $\mathrm{H}$ & -0.69641 & -3.65838 & 2.25989 \\
\hline $\mathrm{H}$ & -1.25245 & 3.06574 & -1.96487 \\
\hline $\mathrm{H}$ & -1.44501 & 4.25801 & -0.63639 \\
\hline $\mathrm{H}$ & -2.82201 & 3.21049 & -1.11742 \\
\hline $\mathrm{H}$ & -3.77803 & 0.69947 & 0.0358 \\
\hline
\end{tabular}




$\begin{array}{rrrr}\mathrm{H} & -4.29035 & -0.96249 & 0.50763 \\ \mathrm{H} & -4.32568 & -0.45490 & -1.21198 \\ \mathrm{H} & 3.07942 & 1.08796 & -1.89954 \\ \mathrm{H} & 3.42923 & 2.40185 & -0.72211 \\ \mathrm{H} & 2.71403 & 2.78458 & -2.32313 \\ \mathrm{H} & 0.05559 & 0.88035 & -3.13049\end{array}$

49

$\begin{array}{lrrr}\mathrm{Cm} & 0.07753 & 0.05826 & 0.05487 \\ \mathrm{O} & -1.92508 & -1.14882 & -1.01297 \\ \mathrm{C} & -2.60585 & -2.45054 & -1.05371 \\ \mathrm{H} & -2.26302 & -0.60107 & -1.73592 \\ \mathrm{O} & 1.79336 & 1.03773 & -1.57350 \\ \mathrm{H} & 2.09923 & 1.95389 & -1.64449 \\ \mathrm{H} & 2.25944 & 0.55121 & -2.26862 \\ \mathrm{O} & 0.31708 & 0.48676 & 2.66694 \\ \mathrm{H} & -0.39016 & 0.65506 & 3.30794 \\ \mathrm{H} & 1.09521 & 0.25843 & 3.19735 \\ \mathrm{O} & 1.06465 & -1.74190 & -1.48087 \\ \mathrm{C} & 0.73234 & -2.42133 & -2.74481 \\ \mathrm{H} & 1.96528 & -1.99532 & -1.23205 \\ \mathrm{O} & 2.37103 & -0.64149 & 0.92637 \\ \mathrm{C} & 3.73327 & -0.08151 & 0.98174 \\ \mathrm{H} & 2.35537 & -1.45551 & 1.44999 \\ \mathrm{O} & -0.08527 & -2.20171 & 1.18982 \\ \mathrm{C} & -0.60890 & -2.79172 & 2.43309 \\ \mathrm{H} & 0.07407 & -2.92171 & 0.56268 \\ \mathrm{O} & -1.20277 & 1.43834 & -1.71237 \\ \mathrm{C} & -1.02493 & 1.84637 & -3.11570 \\ \mathrm{H} & -1.90679 & 1.98138 & -1.32957 \\ \mathrm{O} & 0.59270 & 2.41701 & 0.71242 \\ \mathrm{C} & 0.73636 & 3.75024 & 0.11641 \\ \mathrm{H} & 0.78763 & 2.47767 & 1.65871 \\ \mathrm{O} & -2.13523 & 0.75638 & 1.04340 \\ \mathrm{C} & -2.70947 & 1.73479 & 1.97754 \\ \mathrm{H} & -2.83363 & 0.14369 & 0.77081 \\ \mathrm{H} & -2.37970 & -2.95892 & -0.11439 \\ \mathrm{H} & -2.24579 & -3.03865 & -1.90417 \\ \mathrm{H} & -3.68660 & -2.29547 & -1.13419 \\ \mathrm{H} & -0.23311 & -2.02900 & -3.06977 \\ \mathrm{H} & 0.66913 & -3.50172 & -2.57875 \\ \mathrm{H} & 1.49714 & -2.20075 & -3.49651 \\ \mathrm{H} & 3.72061 & 0.83248 & 0.38553 \\ \mathrm{H} & 4.44394 & -0.79913 & 0.55899 \\ \mathrm{H} & 3.99898 & 0.14698 & 2.01925 \\ \mathrm{H} & -0.77442 & -1.96759 & 3.12884 \\ \mathrm{H} & 0.12642 & -3.49080 & 2.84448 \\ \mathrm{H} & -1.55327 & -3.30872 & 2.23292 \\ \mathrm{H} & -0.31038 & 1.15017 & -3.56000 \\ \mathrm{H} & -0.63791 & 2.86957 & -3.16515 \\ \mathrm{H} & -1.98420 & 1.78256 & -3.63972 \\ \mathrm{H} & 0.42430 & 3.66789 & -0.92777\end{array}$




$\begin{array}{rrrr}\mathrm{H} & 1.77963 & 4.07812 & 0.18437 \\ \mathrm{H} & 0.08559 & 4.46063 & 0.63627 \\ \mathrm{H} & -1.93441 & 2.47935 & 2.17502 \\ \mathrm{H} & -3.01530 & 1.23344 & 2.90281 \\ \mathrm{H} & -3.57478 & 2.22136 & 1.51584\end{array}$

46

$\begin{array}{lrrr}\mathrm{Cm} & 0.11590 & -0.00848 & -0.20681 \\ \mathrm{O} & -1.64829 & 1.58211 & -1.00938 \\ \mathrm{C} & -2.26233 & 2.87830 & -0.68429 \\ \mathrm{H} & -2.07928 & 1.22729 & -1.80085 \\ \mathrm{O} & -1.08792 & -2.20276 & -0.15079 \\ \mathrm{C} & -1.04612 & -3.58020 & -0.66306 \\ \mathrm{H} & -1.82472 & -2.12844 & 0.47229 \\ \mathrm{O} & 2.60437 & 0.34330 & 0.29730 \\ \mathrm{C} & 3.78598 & 1.11907 & -0.11076 \\ \mathrm{H} & 2.84730 & -0.18455 & 1.07016 \\ \mathrm{O} & -1.90213 & -0.14717 & 1.53843 \\ \mathrm{C} & -3.34101 & 0.18132 & 1.56772 \\ \mathrm{H} & -1.66576 & -0.49640 & 2.40944 \\ \mathrm{O} & 0.90951 & -1.11213 & 1.98560 \\ \mathrm{C} & 1.14262 & -2.50766 & 2.40708 \\ \mathrm{H} & 1.04942 & -0.53999 & 2.75425 \\ \mathrm{O} & 0.20183 & 1.85555 & 1.43848 \\ \mathrm{C} & 1.07899 & 2.99909 & 1.74732 \\ \mathrm{H} & -0.59930 & 1.93155 & 1.97603 \\ \mathrm{O} & -1.00138 & -0.61904 & -2.58192 \\ \mathrm{H} & -1.63559 & -1.33271 & -2.74926 \\ \mathrm{H} & -0.74572 & -0.29002 & -3.45780 \\ \mathrm{O} & 1.61018 & -1.56262 & -1.54234 \\ \mathrm{H} & 1.39533 & -2.08191 & -2.33152 \\ \mathrm{H} & 2.55288 & -1.70762 & -1.37267 \\ \mathrm{O} & 1.07122 & 1.49373 & -2.02774 \\ \mathrm{H} & 1.88487 & 1.39058 & -2.54424 \\ \mathrm{H} & 0.68374 & 2.33618 & -2.30953 \\ \mathrm{H} & -1.66691 & 3.32173 & 0.11596 \\ \mathrm{H} & -3.29553 & 2.72855 & -0.35522 \\ \mathrm{H} & -2.23839 & 3.52582 & -1.56678 \\ \mathrm{H} & -0.10510 & -3.69554 & -1.20452 \\ \mathrm{H} & -1.89845 & -3.75570 & -1.32831 \\ \mathrm{H} & -1.07533 & -4.28362 & 0.17528 \\ \mathrm{H} & 3.43991 & 1.91366 & -0.77560 \\ \mathrm{H} & 4.49991 & 0.46931 & -0.62811 \\ \mathrm{H} & 4.25602 & 1.56266 & 0.77290 \\ \mathrm{H} & -3.64334 & 0.36276 & 0.53462 \\ \mathrm{H} & -3.50919 & 1.07311 & 2.18046 \\ \mathrm{H} & -3.90292 & -0.66482 & 1.97620 \\ \mathrm{H} & 1.12799 & -3.11474 & 1.49976 \\ \mathrm{H} & 0.35233 & -2.82583 & 3.09478 \\ \mathrm{H} & 1.12027 & -2.58477 & 2.89338 \\ \mathrm{H} & 1.93584 & 2.95111 & 1.04947 \\ \mathrm{H} & & 2.91840 & 2.77901 \\ & & & \end{array}$


46

$\begin{array}{lrrr}\mathrm{Cm} & 0.15803 & -0.01499 & -0.07346 \\ \mathrm{O} & 0.11155 & 2.51624 & -0.29078 \\ \mathrm{C} & 0.66601 & 3.73462 & 0.31930 \\ \mathrm{H} & -0.59377 & 2.77555 & -0.89999 \\ \mathrm{O} & -1.96447 & -1.34022 & 0.07431 \\ \mathrm{C} & -2.66366 & -2.48189 & -0.53198 \\ \mathrm{H} & -2.55130 & -0.93627 & 0.72817 \\ \mathrm{O} & 2.43972 & -1.18769 & 0.00823 \\ \mathrm{C} & 3.77800 & -1.15586 & -0.60779 \\ \mathrm{H} & 2.41850 & -1.91581 & 0.64532 \\ \mathrm{O} & -1.41584 & 0.80633 & 1.78281 \\ \mathrm{C} & -2.30492 & 1.96018 & 2.00933 \\ \mathrm{H} & -1.34742 & 0.31957 & 2.61669 \\ \mathrm{O} & 0.40416 & -1.62405 & 1.91840 \\ \mathrm{C} & -0.13911 & -2.93384 & 2.32431 \\ \mathrm{H} & 0.92862 & -1.27258 & 2.65246 \\ \mathrm{O} & 1.67991 & 0.96005 & 1.77636 \\ \mathrm{H} & 2.63971 & 0.82606 & 1.80190 \\ \mathrm{H} & 1.49951 & 1.68148 & 2.39822 \\ \mathrm{O} & -1.33407 & 0.79325 & -1.98162 \\ \mathrm{C} & -2.76509 & 0.84546 & -2.32520 \\ \mathrm{H} & -0.82368 & 1.00748 & -2.77635 \\ \mathrm{O} & 0.31222 & -1.85012 & -1.84058 \\ \mathrm{H} & -0.31787 & -2.07258 & -2.54265 \\ \mathrm{H} & 1.00725 & -2.52354 & -1.88681 \\ \mathrm{O} & 1.54687 & 0.85129 & -2.08058 \\ \mathrm{H} & 2.00281 & 0.34446 & -2.76973 \\ \mathrm{H} & 1.85699 & 1.76524 & -2.17073 \\ \mathrm{H} & 1.67294 & 3.49348 & 0.66790 \\ \mathrm{H} & 0.03133 & 4.05935 & 1.15105 \\ \mathrm{H} & 0.72535 & 4.52420 & -0.43644 \\ \mathrm{H} & -1.90366 & -3.13684 & -0.96321 \\ \mathrm{H} & -3.35493 & -2.12826 & -1.30404 \\ \mathrm{H} & -3.21243 & -3.02485 & 0.24392 \\ \mathrm{H} & 3.88607 & -0.18221 & -1.09052 \\ \mathrm{H} & 3.87309 & -1.96361 & -1.34184 \\ \mathrm{H} & 4.54038 & -1.26772 & 0.17007 \\ \mathrm{H} & -2.44945 & 2.44394 & 1.04114 \\ \mathrm{H} & -1.84183 & 2.65366 & 2.71846 \\ \mathrm{H} & -3.26672 & 1.60983 & 2.39727 \\ \mathrm{H} & -0.55947 & -3.39262 & 1.42773 \\ \mathrm{H} & -0.91494 & -2.79645 & 3.08464 \\ \mathrm{H} & -3.31944 & -3.55713 & 2.71899 \\ \mathrm{H} & -3.00064 & 0.67599 & -1.39999 \\ & & 0.06870 & -3.05989 \\ \mathrm{H} & & 1.83306 & -2.73016\end{array}$




$\begin{array}{lrrr}\mathrm{Cm} & 0.05269 & 0.06511 & -0.04417 \\ \mathrm{O} & -2.31322 & 0.89032 & -0.57915 \\ \mathrm{C} & -3.52081 & 1.37505 & 0.11085 \\ \mathrm{H} & -2.54825 & 0.70067 & -1.49889 \\ \mathrm{O} & 0.46756 & -2.14623 & -1.20487 \\ \mathrm{C} & 1.40722 & -2.88276 & -2.06182 \\ \mathrm{H} & -0.21601 & -2.75999 & -0.90007 \\ \mathrm{O} & 1.68215 & 1.32305 & 1.47361 \\ \mathrm{C} & 1.98814 & 2.69435 & 1.91836 \\ \mathrm{H} & 2.24045 & 0.71315 & 1.97578 \\ \mathrm{O} & -1.60067 & -1.87462 & 0.60799 \\ \mathrm{C} & -2.96455 & -2.31837 & 0.26729 \\ \mathrm{H} & -1.30389 & -2.40148 & 1.36358 \\ \mathrm{O} & 1.18674 & -1.43309 & 1.71426 \\ \mathrm{C} & 2.18602 & -2.51783 & 1.66692 \\ \mathrm{H} & 0.88177 & -1.34724 & 2.62919 \\ \mathrm{O} & -1.00900 & 0.60086 & 2.21425 \\ \mathrm{H} & -0.68061 & 1.26486 & 2.83938 \\ \mathrm{H} & -1.87265 & 0.32179 & 2.55413 \\ \mathrm{O} & -0.44914 & 0.14512 & -2.59918 \\ \mathrm{H} & -0.48377 & -0.63537 & -3.17269 \\ \mathrm{H} & -0.45274 & 0.91143 & -3.19312 \\ \mathrm{O} & 2.29916 & 0.30416 & -1.17220 \\ \mathrm{H} & 2.52842 & 0.18868 & -2.10669 \\ \mathrm{H} & 3.09610 & 0.65229 & -0.74501 \\ \mathrm{O} & -0.00812 & 2.46440 & -0.77176 \\ \mathrm{C} & 0.88036 & 3.51907 & -1.28319 \\ \mathrm{H} & -0.91950 & 2.78725 & -0.81383 \\ \mathrm{H} & -3.18950 & 1.86818 & 1.02773 \\ \mathrm{H} & -4.18547 & 0.53516 & 0.33788 \\ \mathrm{H} & -4.03627 & 2.10071 & -0.52638 \\ \mathrm{H} & 2.26120 & -2.22764 & -2.24604 \\ \mathrm{H} & 0.91974 & -3.15032 & -3.00555 \\ \mathrm{H} & 1.74469 & -3.78731 & -1.54562 \\ \mathrm{H} & 1.17955 & 3.33294 & 1.55879 \\ \mathrm{H} & 2.94740 & 3.01624 & 1.50003 \\ \mathrm{H} & 2.02941 & 2.72172 & 3.01218 \\ \mathrm{H} & -3.23385 & -1.82599 & -0.66959 \\ \mathrm{H} & -3.66055 & -2.03600 & 1.06404 \\ \mathrm{H} & -2.97341 & -3.40439 & 0.12947 \\ \mathrm{H} & 1.64907 & -2.47427 & 0.67938 \\ \mathrm{H} & 0.939171 & -3.48402 & 1.81968 \\ \mathrm{H} & -2.35213 & 2.44127 \\ \mathrm{H} & \mathrm{H} & 3.14350 & -1.20071 \\ \mathrm{H} & -.96171 & 3.72812 & -2.33085\end{array}$




$\begin{array}{lrrr}\mathrm{Cm} & 0.14956 & -0.00165 & -0.24409 \\ \mathrm{O} & -1.81657 & 1.53048 & 0.01407 \\ \mathrm{C} & -2.22276 & 2.86340 & 0.48003 \\ \mathrm{H} & -2.60040 & 1.06254 & -0.30944 \\ \mathrm{O} & -0.25535 & -2.16850 & 0.92778 \\ \mathrm{C} & -0.25939 & -3.62189 & 0.70304 \\ \mathrm{H} & -0.49167 & -2.00471 & 1.85110 \\ \mathrm{O} & 2.27729 & 0.47103 & -1.71517 \\ \mathrm{H} & 2.34619 & 0.99150 & -2.52965 \\ \mathrm{H} & 3.19005 & 0.32417 & -1.42557 \\ \mathrm{O} & -0.23654 & 0.26680 & 2.31683 \\ \mathrm{C} & -1.34745 & 0.60075 & 3.22956 \\ \mathrm{H} & 0.54939 & 0.09346 & 2.85499 \\ \mathrm{O} & 2.31355 & -0.49378 & 0.99963 \\ \mathrm{C} & 3.07572 & -1.69684 & 1.38301 \\ \mathrm{H} & 2.82910 & 0.28543 & 1.25332 \\ \mathrm{O} & 1.12425 & 2.18409 & 0.54867 \\ \mathrm{C} & 1.78465 & 3.37320 & -0.01889 \\ \mathrm{H} & 0.84839 & 2.40017 & 1.45077 \\ \mathrm{O} & -2.11660 & -0.94260 & -1.15753 \\ \mathrm{C} & -3.16526 & -1.88128 & -0.72134 \\ \mathrm{H} & -2.27455 & -0.73420 & -2.08955 \\ \mathrm{O} & 0.66003 & -1.78121 & -1.98319 \\ \mathrm{H} & 0.13056 & -2.53444 & -2.28453 \\ \mathrm{H} & 1.50215 & -1.83312 & -2.46016 \\ \mathrm{O} & -0.43739 & 1.06287 & -2.55186 \\ \mathrm{H} & -0.22590 & 0.76749 & -3.45100 \\ \mathrm{H} & -0.91579 & 1.89918 & -2.66086 \\ \mathrm{H} & -1.31686 & 3.38369 & 0.79618 \\ \mathrm{H} & -2.91719 & 2.76702 & 1.32072 \\ \mathrm{H} & -2.69776 & 3.41046 & -0.34081 \\ \mathrm{H} & 0.29523 & -3.80852 & -0.21868 \\ \mathrm{H} & -1.28934 & -3.98106 & 0.61523 \\ \mathrm{H} & 0.24412 & -4.11931 & 1.53797 \\ \mathrm{H} & -2.25607 & 0.61662 & 2.62507 \\ \mathrm{H} & -1.17234 & 1.58025 & 3.68619 \\ \mathrm{H} & -1.42780 & -0.16685 & 4.00591 \\ \mathrm{H} & 2.51426 & -2.55489 & 1.00964 \\ \mathrm{H} & 3.16630 & -1.74464 & 2.47309 \\ \mathrm{H} & 4.06823 & -1.66704 & 0.92185 \\ \mathrm{H} & 2.09864 & 3.10820 & -1.02970 \\ \mathrm{H} & 2.65677 & 3.63177 & 0.59020 \\ \mathrm{H} & -3.07908 & 4.20996 & -0.05088 \\ \mathrm{H} & -3.04852 & -2.01018 & 0.35633 \\ \mathrm{H} & -4.15060 & -2.83960 & -1.23802 \\ \mathrm{H} & & -1.45589 & -0.93720 \\ & & & \end{array}$




\begin{tabular}{|c|c|c|c|}
\hline $\mathrm{Cm}$ & -0.18879 & 0.00426 & 0.09456 \\
\hline 0 & -0.85507 & -1.80497 & -1.49368 \\
\hline C & -0.75781 & -3.25700 & -1.70174 \\
\hline $\mathrm{H}$ & -1.39329 & -1.42851 & -2.20570 \\
\hline O & 0.97838 & 1.49214 & -1.51255 \\
\hline C & 0.96722 & 2.83612 & -2.11104 \\
\hline $\mathrm{H}$ & 1.76928 & 1.02403 & -1.81770 \\
\hline 0 & -0.76041 & 0.27368 & 2.81738 \\
\hline $\mathrm{H}$ & -1.52710 & -0.08003 & 3.29408 \\
\hline $\mathrm{H}$ & -0.10845 & 0.45862 & 3.51036 \\
\hline O & 2.09135 & -0.97743 & -0.91721 \\
\hline C & 2.51456 & -1.79741 & -2.07027 \\
\hline $\mathrm{H}$ & 2.88330 & -0.75386 & -0.40780 \\
\hline O & 1.80807 & 0.75836 & 1.45312 \\
\hline $\mathrm{C}$ & 2.49425 & 2.05859 & 1.57821 \\
\hline $\mathrm{H}$ & 2.19975 & 0.14890 & 2.09499 \\
\hline 0 & 0.52390 & -2.06954 & 1.27012 \\
\hline C & 0.03098 & -2.96666 & 2.32941 \\
\hline $\mathrm{H}$ & 1.29888 & -2.47937 & 0.86025 \\
\hline O & -1.81104 & 0.75770 & -1.87108 \\
\hline $\mathrm{H}$ & -1.54756 & 1.24175 & -2.66850 \\
\hline $\mathrm{H}$ & -2.77886 & 0.81232 & -1.84463 \\
\hline O & -1.20429 & 2.12236 & 0.86953 \\
\hline C & -1.79261 & 3.39397 & 0.42310 \\
\hline $\mathrm{H}$ & -1.19782 & 2.10475 & 1.83933 \\
\hline O & -2.59011 & -0.68993 & 0.68522 \\
\hline $\mathrm{H}$ & -3.29653 & -0.16013 & 1.08566 \\
\hline $\mathrm{H}$ & -2.99998 & -1.53834 & 0.45663 \\
\hline $\mathrm{H}$ & -0.15218 & -3.65764 & -0.88696 \\
\hline $\mathrm{H}$ & -0.28004 & -3.46074 & -2.66513 \\
\hline $\mathrm{H}$ & -1.75895 & -3.69947 & -1.67684 \\
\hline $\mathrm{H}$ & 0.08671 & 3.35221 & -1.72462 \\
\hline $\mathrm{H}$ & 0.91452 & 2.75094 & -3.20164 \\
\hline $\mathrm{H}$ & 1.87407 & 3.37511 & -1.81892 \\
\hline $\mathrm{H}$ & 1.67803 & -1.80834 & -2.77167 \\
\hline $\mathrm{H}$ & 2.75393 & -2.81334 & -1.73958 \\
\hline $\mathrm{H}$ & 3.39059 & -1.34146 & -2.54255 \\
\hline $\mathrm{H}$ & 1.98669 & 2.74457 & 0.89737 \\
\hline $\mathrm{H}$ & 3.54612 & 1.94831 & 1.29571 \\
\hline $\mathrm{H}$ & 2.41313 & 2.42076 & 2.60807 \\
\hline $\mathrm{H}$ & -0.93131 & -2.57052 & 2.65871 \\
\hline $\mathrm{H}$ & 0.74339 & -2.98015 & 3.16040 \\
\hline $\mathrm{H}$ & -0.10122 & -3.97472 & 1.92361 \\
\hline $\mathrm{H}$ & -2.00751 & 3.29213 & -0.64247 \\
\hline $\mathrm{H}$ & -1.08362 & 4.20940 & 0.59912 \\
\hline $\mathrm{H}$ & -2.72287 & 3.57541 & 0.97014 \\
\hline
\end{tabular}




$\begin{array}{lrrr}\mathrm{Cm} & 0.08849 & -0.08312 & 0.23090 \\ \mathrm{O} & -1.16920 & -2.28286 & 0.02567 \\ \mathrm{C} & -0.98049 & -3.73758 & 0.13510 \\ \mathrm{H} & -2.04070 & -2.11713 & -0.36050 \\ \mathrm{O} & 0.04158 & 1.46496 & -1.71819 \\ \mathrm{C} & -0.19199 & 2.86919 & -2.08576 \\ \mathrm{H} & 0.27686 & 0.97519 & -2.51845 \\ \mathrm{O} & 1.36117 & 0.27027 & 2.59127 \\ \mathrm{H} & 1.04915 & 0.02199 & 3.47520 \\ \mathrm{H} & 2.26445 & 0.59780 & 2.72161 \\ \mathrm{O} & 0.90967 & -1.15763 & -1.97658 \\ \mathrm{C} & 0.39004 & -2.09975 & -2.98484 \\ \mathrm{H} & 1.83885 & -0.98491 & -2.18691 \\ \mathrm{O} & 2.47712 & 0.66174 & -0.09316 \\ \mathrm{C} & 3.15654 & 1.89902 & -0.51731 \\ \mathrm{H} & 3.15071 & -0.00274 & 0.11122 \\ \mathrm{O} & 1.75223 & -1.97116 & 0.83151 \\ \mathrm{H} & 2.09989 & -2.14540 & 1.71946 \\ \mathrm{H} & 1.96197 & -2.75924 & 0.30766 \\ \mathrm{O} & -2.36893 & 0.21341 & -0.38260 \\ \mathrm{C} & -3.16103 & 0.62121 & -1.55609 \\ \mathrm{H} & -2.95728 & 0.19957 & 0.38632 \\ \mathrm{O} & -0.38346 & 2.11077 & 1.31343 \\ \mathrm{C} & -1.39705 & 3.17791 & 1.29128 \\ \mathrm{H} & 0.23052 & 2.29255 & 2.03965 \\ \mathrm{O} & -1.44045 & -0.56380 & 2.29760 \\ \mathrm{H} & -1.72971 & 0.00585 & 3.02671 \\ \mathrm{H} & -1.79680 & -1.44386 & 2.49383 \\ \mathrm{H} & -0.20683 & -3.90812 & 0.88713 \\ \mathrm{H} & -0.67980 & -4.15047 & -0.83373 \\ \mathrm{H} & -1.91512 & -4.20116 & 0.46632 \\ \mathrm{H} & -0.16666 & 3.45167 & -1.16416 \\ \mathrm{H} & -1.16596 & 2.96606 & -2.57566 \\ \mathrm{H} & 0.60410 & 3.20458 & -2.75758 \\ \mathrm{H} & -0.67828 & -2.21387 & -2.78978 \\ \mathrm{H} & 0.90152 & -3.06331 & -2.89277 \\ \mathrm{H} & 0.54399 & -1.68641 & -3.98675 \\ \mathrm{H} & 2.38353 & 2.66221 & -0.62108 \\ \mathrm{H} & 3.66228 & 1.73368 & -1.47408 \\ \mathrm{H} & 3.88023 & 2.19974 & 0.24697 \\ \mathrm{H} & -2.50866 & 0.52644 & -2.42620 \\ \mathrm{H} & -3.49479 & 1.65729 & -1.43939 \\ \mathrm{H} & -4.02403 & -0.04363 & -1.66151 \\ \mathrm{H} & -2.07668 & 2.95192 & 0.46763 \\ \mathrm{H} & -0.90945 & 4.14420 & 1.12748 \\ \mathrm{H} & -1.94189 & 3.18765 & 2.24085\end{array}$




\begin{tabular}{|c|c|c|c|}
\hline $\mathrm{Cm}$ & 0.12852 & 0.00184 & 0.16443 \\
\hline 0 & -1.10091 & 2.11076 & -0.61992 \\
\hline C & -2.03400 & 3.13946 & -0.13143 \\
\hline $\mathrm{H}$ & -1.05914 & 2.17310 & -1.58472 \\
\hline 0 & -0.34760 & -2.07006 & -1.16291 \\
\hline C & 0.30906 & -3.16425 & -1.89218 \\
\hline $\mathrm{H}$ & -1.30452 & -2.19770 & -1.22080 \\
\hline 0 & 1.78327 & 0.12162 & 2.21359 \\
\hline $\mathrm{H}$ & 2.44551 & 0.80086 & 2.41189 \\
\hline $\mathrm{H}$ & 1.85516 & -0.52765 & 2.92934 \\
\hline O & -2.37600 & -0.60808 & 0.07337 \\
\hline C & -3.59318 & -0.19221 & -0.64646 \\
\hline $\mathrm{H}$ & -2.63575 & -1.25171 & 0.74826 \\
\hline O & -0.33437 & -1.81866 & 1.88073 \\
\hline $\mathrm{C}$ & -0.11726 & -3.27419 & 1.97397 \\
\hline $\mathrm{H}$ & -0.72039 & -1.51603 & 2.71532 \\
\hline 0 & -0.97036 & 1.00964 & 2.26265 \\
\hline $\mathrm{H}$ & -0.48413 & 1.39078 & 3.00991 \\
\hline $\mathrm{H}$ & -1.89605 & 1.26613 & 2.39238 \\
\hline 0 & 0.59686 & 0.57680 & -2.27845 \\
\hline C & 0.23908 & 0.10144 & -3.62663 \\
\hline $\mathrm{H}$ & 1.30070 & 1.23577 & -2.36755 \\
\hline O & 2.38432 & -1.01985 & -0.35251 \\
\hline $\mathrm{H}$ & 2.76522 & -1.24151 & -1.21545 \\
\hline $\mathrm{H}$ & 3.05479 & -1.25903 & 0.30487 \\
\hline O & 1.63106 & 2.05441 & 0.01896 \\
\hline C & 3.03463 & 2.45677 & 0.19156 \\
\hline $\mathrm{H}$ & 1.09376 & 2.85367 & -0.07972 \\
\hline $\mathrm{H}$ & -1.81450 & 3.29591 & 0.92721 \\
\hline $\mathrm{H}$ & -3.06743 & 2.80569 & -0.26967 \\
\hline $\mathrm{H}$ & -1.86368 & 4.07243 & -0.67813 \\
\hline $\mathrm{H}$ & 1.34761 & -3.20046 & -1.55713 \\
\hline $\mathrm{H}$ & 0.25674 & -2.97779 & -2.96947 \\
\hline $\mathrm{H}$ & -0.18460 & -4.11046 & -1.64910 \\
\hline $\mathrm{H}$ & -3.27056 & 0.44866 & -1.46960 \\
\hline $\mathrm{H}$ & -4.25695 & 0.35576 & 0.03006 \\
\hline $\mathrm{H}$ & -4.10525 & -1.07527 & -1.04195 \\
\hline $\mathrm{H}$ & 0.41527 & -3.57014 & 1.06862 \\
\hline $\mathrm{H}$ & -1.08183 & -3.78854 & 2.03537 \\
\hline $\mathrm{H}$ & 0.48856 & -3.49960 & 2.85754 \\
\hline $\mathrm{H}$ & -0.62226 & -0.55895 & -3.50962 \\
\hline $\mathrm{H}$ & 1.08223 & -0.44119 & -4.06631 \\
\hline $\mathrm{H}$ & -0.02803 & 0.95631 & -4.25600 \\
\hline $\mathrm{H}$ & 3.63171 & 1.54159 & 0.18424 \\
\hline $\mathrm{H}$ & 3.15599 & 2.99670 & 1.13785 \\
\hline $\mathrm{H}$ & 3.33961 & 3.09836 & -0.64160 \\
\hline
\end{tabular}




$\begin{array}{lrrr}\mathrm{Cm} & -0.08103 & -0.05001 & 0.07760 \\ \mathrm{O} & -0.59915 & -2.25863 & -1.07944 \\ \mathrm{C} & -0.55978 & -3.71058 & -0.83810 \\ \mathrm{H} & -0.81049 & -2.11365 & -2.01314 \\ \mathrm{O} & 1.48505 & 1.10199 & -1.48809 \\ \mathrm{C} & 1.69859 & 2.31383 & -2.29332 \\ \mathrm{H} & 2.29235 & 0.56736 & -1.51292 \\ \mathrm{O} & -1.14815 & 0.64139 & 2.53268 \\ \mathrm{H} & -2.05672 & 0.49174 & 2.83638 \\ \mathrm{H} & -0.68378 & 0.99970 & 3.30462 \\ \mathrm{O} & 2.24028 & -1.26254 & -0.30401 \\ \mathrm{C} & 2.80226 & -2.31008 & -1.17621 \\ \mathrm{H} & 2.91291 & -1.03746 & 0.35430 \\ \mathrm{O} & 1.64992 & 0.90897 & 1.65124 \\ \mathrm{C} & 2.38715 & 2.18586 & 1.68254 \\ \mathrm{H} & 1.89565 & 0.39910 & 2.43640 \\ \mathrm{O} & 0.29051 & -1.78446 & 1.90204 \\ \mathrm{H} & -0.23894 & -1.86272 & 2.71027 \\ \mathrm{H} & 0.86252 & -2.56634 & 1.87862 \\ \mathrm{O} & -1.12577 & 0.23124 & -2.28333 \\ \mathrm{H} & -0.66649 & 0.54697 & -3.07635 \\ \mathrm{H} & -2.07092 & 0.24936 & -2.49899 \\ \mathrm{O} & -1.03469 & 2.22550 & 0.20613 \\ \mathrm{C} & -1.50816 & 3.33623 & -0.63534 \\ \mathrm{H} & -1.19130 & 2.45924 & 1.13279 \\ \mathrm{O} & -2.52630 & -0.62781 & 0.22630 \\ \mathrm{C} & -3.80865 & -0.02224 & 0.61281 \\ \mathrm{H} & -2.68981 & -1.54539 & -0.03450 \\ \mathrm{H} & -0.56215 & -3.85490 & 0.24454 \\ \mathrm{H} & 0.34274 & -4.13988 & -1.28393 \\ \mathrm{H} & -1.45156 & -4.17532 & -1.27096 \\ \mathrm{H} & 0.78166 & 2.90296 & -2.23793 \\ \mathrm{H} & 1.90881 & 2.03223 & -3.33065 \\ \mathrm{H} & 2.53606 & 2.88621 & -1.88167 \\ \mathrm{H} & 2.11579 & -2.42154 & -2.01829 \\ \mathrm{H} & 2.88991 & -3.25107 & -0.62321 \\ \mathrm{H} & 3.78609 & -1.99626 & -1.53970 \\ \mathrm{H} & 2.03281 & 2.77036 & 0.83137 \\ \mathrm{H} & 3.46124 & 1.99374 & 1.59405 \\ \mathrm{H} & 2.17128 & 2.71264 & 2.61760 \\ \mathrm{H} & -1.51208 & 2.97692 & -1.66608 \\ \mathrm{H} & -0.83476 & 4.19221 & -0.52559 \\ \mathrm{H} & -2.52437 & 3.61137 & -0.33679 \\ \mathrm{H} & -3.61812 & 1.03847 & 0.79250 \\ \mathrm{H} & -4.19307 & -0.50925 & 1.51627 \\ \mathrm{H} & -4.53012 & -0.13243 & -0.20328 \\ & & & \end{array}$




$\begin{array}{lrrr}\mathrm{Cm} & 0.08334 & 0.07538 & 0.12226 \\ \mathrm{O} & -1.83245 & -1.45233 & 0.74120 \\ \mathrm{C} & -2.17523 & -2.86140 & 0.98211 \\ \mathrm{H} & -2.64738 & -0.93004 & 0.75558 \\ \mathrm{O} & -0.18948 & 1.71407 & -1.81744 \\ \mathrm{H} & -0.08615 & 2.67700 & -1.80570 \\ \mathrm{H} & -0.45073 & 1.49029 & -2.72247 \\ \mathrm{O} & 2.21673 & 0.11894 & 1.66778 \\ \mathrm{H} & 2.24008 & -0.02579 & 2.62615 \\ \mathrm{H} & 3.13396 & 0.02444 & 1.36891 \\ \mathrm{O} & -0.50484 & -1.14125 & -2.04147 \\ \mathrm{C} & -1.70282 & -1.73050 & -2.66779 \\ \mathrm{H} & 0.21504 & -1.17144 & -2.68839 \\ \mathrm{O} & 2.19303 & -0.08684 & -1.26671 \\ \mathrm{C} & 3.05022 & 0.79946 & -2.07482 \\ \mathrm{H} & 2.61805 & -0.95481 & -1.21082 \\ \mathrm{O} & 1.02414 & -2.29056 & 0.19043 \\ \mathrm{C} & 1.78110 & -3.18993 & 1.07910 \\ \mathrm{H} & 0.63093 & -2.83102 & -0.50987 \\ \mathrm{O} & -2.18637 & 1.26998 & 0.40182 \\ \mathrm{C} & -3.20822 & 1.85139 & -0.48570 \\ \mathrm{H} & -2.35826 & 1.58405 & 1.30128 \\ \mathrm{O} & 0.79465 & 2.37013 & 0.86495 \\ \mathrm{C} & 0.35535 & 3.76845 & 0.76840 \\ \mathrm{H} & 1.65887 & 2.34871 & 1.30100 \\ \mathrm{O} & -0.50646 & 0.26778 & 2.64903 \\ \mathrm{H} & -0.28814 & 0.95873 & 3.29330 \\ \mathrm{H} & -1.01203 & -0.39900 & 3.13891 \\ \mathrm{H} & -1.24096 & -3.38806 & 1.18381 \\ \mathrm{H} & -2.67129 & -3.28206 & 0.10106 \\ \mathrm{H} & -2.83249 & -2.93285 & 1.85465 \\ \mathrm{H} & -2.51686 & -1.62627 & -1.94825 \\ \mathrm{H} & -1.52154 & -2.78768 & -2.88710 \\ \mathrm{H} & -1.94228 & -1.18933 & -3.58884 \\ \mathrm{H} & 2.55350 & 1.77050 & -2.10912 \\ \mathrm{H} & 3.15257 & 0.39074 & -3.08536 \\ \mathrm{H} & 4.03354 & 0.89347 & -1.60242 \\ \mathrm{H} & 2.16386 & -2.57901 & 1.89823 \\ \mathrm{H} & 2.60919 & -3.64227 & 0.52352 \\ \mathrm{H} & 1.11960 & -3.96872 & 1.47259 \\ \mathrm{H} & -3.08698 & 1.37744 & -1.46220 \\ \mathrm{H} & -3.06383 & 2.93364 & -0.56790 \\ \mathrm{H} & -4.20425 & 1.63338 & -0.08710 \\ \mathrm{H} & -0.66454 & 3.75539 & 0.37507 \\ \mathrm{H} & 1.02312 & 4.32426 & 0.10053 \\ \mathrm{H} & 0.35687 & 4.22623 & 1.76285 \\ & & & \end{array}$




\begin{tabular}{|c|c|c|c|}
\hline $\mathrm{Cm}$ & -0.17334 & 0.00798 & 0.15220 \\
\hline 0 & 1.22005 & -1.21524 & -1.62558 \\
\hline C & 2.49789 & -1.11471 & -2.34718 \\
\hline $\mathrm{H}$ & 0.65220 & -1.84229 & -2.09585 \\
\hline O & -2.54203 & 0.76293 & -0.42110 \\
\hline $\mathrm{H}$ & -3.33719 & 0.54819 & 0.08881 \\
\hline $\mathrm{H}$ & -2.83849 & 1.31675 & -1.15816 \\
\hline O & 0.47276 & 0.37429 & 2.63227 \\
\hline $\mathrm{H}$ & 0.90388 & -0.23110 & 3.25421 \\
\hline $\mathrm{H}$ & 0.43084 & 1.22933 & 3.08552 \\
\hline O & -0.14312 & 1.51356 & -1.88488 \\
\hline C & -0.10665 & 1.40524 & -3.35353 \\
\hline $\mathrm{H}$ & -0.28880 & 2.44255 & -1.65448 \\
\hline O & -0.38303 & 2.47073 & 0.86711 \\
\hline $\mathrm{C}$ & -1.41515 & 3.41857 & 1.32731 \\
\hline $\mathrm{H}$ & 0.47853 & 2.90737 & 0.93363 \\
\hline O & 2.08188 & 1.14839 & 0.09394 \\
\hline C & 3.32078 & 1.15237 & 0.89302 \\
\hline $\mathrm{H}$ & 2.28331 & 1.54380 & -0.76645 \\
\hline 0 & -1.36449 & -2.00509 & -0.93577 \\
\hline C & -2.47052 & -2.22804 & -1.88566 \\
\hline $\mathrm{H}$ & -1.14085 & -2.85674 & -0.53302 \\
\hline O & -1.94453 & -0.87848 & 1.81825 \\
\hline $\mathrm{H}$ & -2.60351 & -1.58035 & 1.70738 \\
\hline $\mathrm{H}$ & -1.97968 & -0.63139 & 2.75478 \\
\hline O & 0.92395 & -2.08272 & 1.01952 \\
\hline C & 1.00855 & -2.90443 & 2.23557 \\
\hline $\mathrm{H}$ & 1.59099 & -2.39373 & 0.39023 \\
\hline $\mathrm{H}$ & 3.20959 & -0.63380 & -1.67290 \\
\hline $\mathrm{H}$ & 2.37043 & -0.52283 & -3.25954 \\
\hline $\mathrm{H}$ & 2.85466 & -2.11919 & -2.59649 \\
\hline $\mathrm{H}$ & -0.05702 & 0.34100 & -3.59170 \\
\hline $\mathrm{H}$ & 0.77617 & 1.92499 & -3.73974 \\
\hline $\mathrm{H}$ & -1.01676 & 1.84044 & -3.77849 \\
\hline $\mathrm{H}$ & 3.06131 & 0.75965 & 1.87747 \\
\hline $\mathrm{H}$ & 3.69321 & 2.17787 & 0.98219 \\
\hline $\mathrm{H}$ & 4.07340 & 0.51460 & 0.41770 \\
\hline $\mathrm{H}$ & -2.36935 & 2.89059 & 1.28364 \\
\hline $\mathrm{H}$ & -1.43414 & 4.29226 & 0.66743 \\
\hline $\mathrm{H}$ & -1.20212 & 3.72791 & 2.35600 \\
\hline $\mathrm{H}$ & -2.62199 & -1.28810 & -2.42012 \\
\hline $\mathrm{H}$ & -3.37961 & -2.50785 & -1.34318 \\
\hline $\mathrm{H}$ & -2.19265 & -3.01890 & -2.58996 \\
\hline $\mathrm{H}$ & 0.15459 & -2.63510 & 2.86242 \\
\hline $\mathrm{H}$ & 1.95421 & -2.70843 & 2.75357 \\
\hline $\mathrm{H}$ & 0.94267 & -3.96400 & 1.96854 \\
\hline
\end{tabular}




$\begin{array}{lrrr}\mathrm{Cm} & 0.00729 & 0.00566 & 0.00142 \\ \mathrm{O} & 0.00625 & 0.40003 & -2.60561 \\ \mathrm{H} & 0.31747 & 1.17376 & -3.09920 \\ \mathrm{H} & -0.51663 & -0.12016 & -3.23441 \\ \mathrm{O} & -2.17684 & -0.46143 & 1.25678 \\ \mathrm{H} & -2.36781 & -1.20108 & 1.85226 \\ \mathrm{H} & -3.00016 & 0.04471 & 1.19790 \\ \mathrm{O} & 2.29881 & 0.00140 & 1.31170 \\ \mathrm{H} & 3.15094 & -0.35355 & 1.01623 \\ \mathrm{H} & 2.50543 & 0.56438 & 2.07281 \\ \mathrm{O} & -1.72996 & 1.82290 & -0.45454 \\ \mathrm{C} & -2.68874 & 2.20940 & -1.50640 \\ \mathrm{H} & -1.88699 & 2.38988 & 0.31451 \\ \mathrm{O} & -0.04892 & 1.60246 & 1.98617 \\ \mathrm{C} & -0.54873 & 1.60155 & 3.37418 \\ \mathrm{H} & 0.47140 & 2.40825 & 1.85559 \\ \mathrm{O} & 1.20728 & 2.17382 & -0.61933 \\ \mathrm{C} & 2.58470 & 2.64250 & -0.85549 \\ \mathrm{H} & 0.60230 & 2.88095 & -0.88543 \\ \mathrm{O} & -1.19643 & -1.82424 & -1.25557 \\ \mathrm{C} & -2.58291 & -2.23641 & -1.52869 \\ \mathrm{H} & -0.60555 & -2.51313 & -1.59209 \\ \mathrm{O} & 0.31860 & -1.92421 & 1.55249 \\ \mathrm{C} & -0.33736 & -3.19215 & 1.89936 \\ \mathrm{H} & 1.09991 & -1.81590 & 2.11374 \\ \mathrm{O} & 1.72799 & -1.34124 & -1.26098 \\ \mathrm{C} & 2.73696 & -2.38573 & -1.02914 \\ \mathrm{H} & 1.80865 & -1.03419 & -2.17597 \\ \mathrm{H} & 3.24725 & 1.80850 & -0.61691 \\ \mathrm{H} & 2.80063 & 3.49641 & -0.20495 \\ \mathrm{H} & 2.70455 & 2.92714 & -1.90602 \\ \mathrm{H} & -2.53737 & 1.52103 & -2.33994 \\ \mathrm{H} & -2.49196 & 3.23849 & -1.82432 \\ \mathrm{H} & -3.71152 & 2.12047 & -1.12571 \\ \mathrm{H} & -1.11494 & 0.67772 & 3.50281 \\ \mathrm{H} & -1.19776 & 2.46913 & 3.53148 \\ \mathrm{H} & 0.29551 & 1.62884 & 4.07103 \\ \mathrm{H} & -1.16463 & -3.32385 & 1.19758 \\ \mathrm{H} & -0.70471 & -3.14997 & 2.93074 \\ \mathrm{H} & 0.37233 & -4.01815 & 1.78810 \\ \mathrm{H} & -3.22450 & -1.39811 & -1.24960 \\ \mathrm{H} & -2.83436 & -3.12099 & -0.93434 \\ \mathrm{H} & -2.69619 & -2.45482 & -2.59554 \\ \mathrm{H} & 3.55683 & -2.78512 & -0.02831 \\ \mathrm{H} & & -1.95636 & -1.10374 \\ \mathrm{H} & -3.18301 & -1.76994 \\ & & & \end{array}$




\begin{tabular}{|c|c|c|c|}
\hline $\mathrm{Cm}$ & 0.15561 & 0.03027 & -0.11218 \\
\hline 0 & -0.37655 & 2.43117 & -0.67111 \\
\hline $\mathrm{C}$ & -0.34388 & 3.77113 & -0.06007 \\
\hline $\mathrm{H}$ & -0.81784 & 2.50183 & -1.52990 \\
\hline O & -1.77133 & -1.44388 & -0.75976 \\
\hline $\mathrm{C}$ & -2.13865 & -2.63331 & -1.54338 \\
\hline $\mathrm{H}$ & -2.55411 & -1.14023 & -0.27791 \\
\hline 0 & 2.40338 & -0.68935 & 0.92232 \\
\hline $\mathrm{C}$ & 3.83825 & -0.34961 & 0.90333 \\
\hline $\mathrm{H}$ & 2.28257 & -1.43722 & 1.52391 \\
\hline O & -2.08924 & 0.57807 & 1.10284 \\
\hline $\mathrm{C}$ & -3.18737 & 1.55410 & 0.96583 \\
\hline $\mathrm{H}$ & -2.22362 & 0.10531 & 1.93645 \\
\hline O & -0.10789 & -1.59898 & 1.84106 \\
\hline $\mathrm{C}$ & -0.54712 & -3.00308 & 1.96325 \\
\hline $\mathrm{H}$ & 0.03135 & -1.25015 & 2.73361 \\
\hline O & 0.67591 & 1.31538 & 2.02250 \\
\hline $\mathrm{H}$ & 1.55403 & 1.36255 & 2.43075 \\
\hline $\mathrm{H}$ & 0.12583 & 1.95636 & 2.49826 \\
\hline O & -0.54702 & 0.37820 & -2.60609 \\
\hline $\mathrm{H}$ & -1.35500 & 0.04822 & -3.02800 \\
\hline $\mathrm{H}$ & 0.01247 & 0.70836 & -3.32638 \\
\hline O & 1.21327 & -1.83624 & -1.46534 \\
\hline $\mathrm{H}$ & 1.00117 & -2.12021 & -2.36742 \\
\hline $\mathrm{H}$ & 1.95900 & -2.38750 & -1.18429 \\
\hline O & 2.04800 & 1.13646 & -1.43680 \\
\hline $\mathrm{H}$ & 2.84819 & 0.74334 & -1.81742 \\
\hline $\mathrm{H}$ & 2.13106 & 2.09270 & -1.57094 \\
\hline $\mathrm{H}$ & 0.40253 & 3.74192 & 0.73692 \\
\hline $\mathrm{H}$ & -1.33184 & 4.02268 & 0.33932 \\
\hline $\mathrm{H}$ & -0.04387 & 4.50576 & -0.81411 \\
\hline $\mathrm{H}$ & -1.21218 & -3.07512 & -1.91590 \\
\hline $\mathrm{H}$ & -2.78898 & -2.34425 & -2.37598 \\
\hline $\mathrm{H}$ & -2.65351 & -3.35188 & -0.89762 \\
\hline $\mathrm{H}$ & 3.92648 & 0.64819 & 0.46794 \\
\hline $\mathrm{H}$ & 4.38857 & -1.08327 & 0.30431 \\
\hline $\mathrm{H}$ & 4.22402 & -0.33938 & 1.92806 \\
\hline $\mathrm{H}$ & -3.12141 & 1.96604 & -0.0433 \\
\hline $\mathrm{H}$ & -3.07772 & 2.34678 & 1.71312 \\
\hline $\mathrm{H}$ & -4.14740 & 1.04470 & 1.09655 \\
\hline $\mathrm{H}$ & -0.53575 & -3.42359 & 0.95595 \\
\hline $\mathrm{H}$ & -1.55700 & -3.04096 & 2.38446 \\
\hline $\mathrm{H}$ & 0.15158 & -3.54878 & 2.60511 \\
\hline
\end{tabular}




$\begin{array}{lrrr}\mathrm{Cm} & 0.05163 & 0.18637 & 0.02219 \\ \mathrm{O} & -2.19585 & -0.29527 & -1.06468 \\ \mathrm{C} & -3.61504 & 0.08451 & -0.96692 \\ \mathrm{H} & -2.13037 & -1.06491 & -1.64674 \\ \mathrm{O} & 1.66895 & -1.57061 & 0.86904 \\ \mathrm{C} & 3.07990 & -1.97637 & 0.75091 \\ \mathrm{H} & 1.18568 & -2.28562 & 1.30623 \\ \mathrm{O} & 0.84978 & 2.57838 & 0.45698 \\ \mathrm{C} & 0.81115 & 3.90923 & -0.17852 \\ \mathrm{H} & 1.34375 & 2.65852 & 1.28557 \\ \mathrm{O} & -1.07540 & -1.51432 & 1.53833 \\ \mathrm{C} & -2.06951 & -2.59542 & 1.40999 \\ \mathrm{H} & -0.95238 & -1.33448 & 2.48186 \\ \mathrm{O} & 0.80134 & 0.58970 & 2.51376 \\ \mathrm{H} & 1.56153 & 0.14826 & 2.92330 \\ \mathrm{H} & 0.39444 & 1.12190 & 3.21533 \\ \mathrm{O} & -1.72548 & 1.55143 & 1.24481 \\ \mathrm{H} & -1.68780 & 2.51277 & 1.36087 \\ \mathrm{H} & -2.59202 & 1.27907 & 1.58297 \\ \mathrm{O} & 0.23444 & -1.48755 & -1.87840 \\ \mathrm{C} & 0.54783 & -2.91749 & -2.05294 \\ \mathrm{H} & 0.23112 & -1.07269 & -2.75373 \\ \mathrm{O} & 2.33062 & 0.63550 & -1.06641 \\ \mathrm{H} & 2.87521 & 0.03835 & -1.60153 \\ \mathrm{H} & 2.87572 & 1.42199 & -0.91454 \\ \mathrm{O} & -0.25770 & 1.41308 & -2.20161 \\ \mathrm{H} & 0.39423 & 1.91690 & -2.71250 \\ \mathrm{H} & -1.11282 & 1.55789 & -2.63471 \\ \mathrm{H} & -3.64982 & 1.13691 & -0.67553 \\ \mathrm{H} & -4.12035 & -0.54474 & -0.22601 \\ \mathrm{H} & -4.08995 & -0.03551 & -1.94574 \\ \mathrm{H} & 0.06489 & 3.86087 & -0.97454 \\ \mathrm{H} & 1.79666 & 4.15741 & -0.58747 \\ \mathrm{H} & 0.51529 & 4.65893 & 0.56266 \\ \mathrm{H} & 3.66600 & -1.06663 & 0.60472 \\ \mathrm{H} & 3.20201 & -2.65999 & -0.09623 \\ \mathrm{H} & 3.39334 & -2.46720 & 1.67770 \\ \mathrm{H} & -2.12705 & -2.84988 & 0.34962 \\ \mathrm{H} & -3.04244 & -2.25116 & 1.77477 \\ \mathrm{H} & -1.73625 & -3.46597 & 1.98371 \\ \mathrm{H} & 0.47233 & -3.38132 & -1.06727 \\ \mathrm{H} & -0.179098 & -3.03004 & -2.45190 \\ \mathrm{H} & & -3.36945 & -2.73477\end{array}$




$\begin{array}{lrrr}\mathrm{Cm} & 0.06486 & 0.01055 & 0.00545 \\ \mathrm{O} & 0.35921 & 2.11141 & -1.42131 \\ \mathrm{C} & 1.06755 & 3.40139 & -1.38284 \\ \mathrm{H} & -0.29579 & 2.15274 & -2.13207 \\ \mathrm{O} & -2.24800 & -0.93791 & 0.38225 \\ \mathrm{C} & -2.96815 & -2.22161 & 0.46182 \\ \mathrm{H} & -2.87053 & -0.22610 & 0.58815 \\ \mathrm{O} & 2.05990 & -1.14848 & 1.18752 \\ \mathrm{C} & 3.50351 & -1.37238 & 0.97323 \\ \mathrm{H} & 1.83406 & -1.49335 & 2.06343 \\ \mathrm{O} & -1.62681 & 1.73672 & 0.89071 \\ \mathrm{C} & -2.35623 & 2.91374 & 0.38409 \\ \mathrm{H} & -1.72386 & 1.72333 & 1.85387 \\ \mathrm{O} & -0.36010 & -0.42715 & 2.55494 \\ \mathrm{H} & -1.04424 & -1.01412 & 2.91279 \\ \mathrm{H} & 0.06115 & -0.01659 & 3.32634 \\ \mathrm{O} & 1.44193 & 1.66820 & 1.36811 \\ \mathrm{H} & 2.32329 & 1.49633 & 1.73231 \\ \mathrm{H} & 1.27179 & 2.60954 & 1.52450 \\ \mathrm{O} & -1.18582 & -0.08605 & -2.24252 \\ \mathrm{H} & -2.13348 & -0.26292 & -2.34650 \\ \mathrm{H} & -0.79436 & -0.23967 & -3.11688 \\ \mathrm{O} & 0.24529 & -2.42281 & -0.55693 \\ \mathrm{C} & -0.27931 & -3.39576 & -1.52947 \\ \mathrm{H} & 0.94271 & -2.85474 & -0.04386 \\ \mathrm{O} & 1.85651 & -0.22876 & -1.77546 \\ \mathrm{H} & 2.32775 & -1.01609 & -2.08702 \\ \mathrm{H} & 2.20303 & 0.51625 & -2.28942 \\ \mathrm{H} & 2.00438 & 3.23963 & -0.84422 \\ \mathrm{H} & 0.45102 & 4.15265 & -0.87699 \\ \mathrm{H} & 1.28689 & 3.72545 & -2.40518 \\ \mathrm{H} & -2.21302 & -3.00723 & 0.51564 \\ \mathrm{H} & -3.59809 & -2.34959 & -0.42463 \\ \mathrm{H} & -3.58591 & -2.23498 & 1.36552 \\ \mathrm{H} & 3.78509 & -0.80100 & 0.08605 \\ \mathrm{H} & 3.69631 & -2.43988 & 0.82142 \\ \mathrm{H} & 4.06373 & -1.01237 & 1.84257 \\ \mathrm{H} & -2.37526 & 2.83303 & -0.70501 \\ \mathrm{H} & -1.84529 & 3.83130 & 0.69316 \\ \mathrm{H} & -3.37984 & 2.90237 & 0.77184 \\ \mathrm{H} & -1.13903 & -2.93039 & -2.01479 \\ \mathrm{H} & -0.59055 & -4.30521 & -1.00577 \\ \mathrm{H} & 0.49303 & -3.63169 & -2.26891 \\ & & & \end{array}$




$\begin{array}{lrrr}\mathrm{Cm} & 0.18819 & -0.05486 & -0.23400 \\ \mathrm{O} & -1.38179 & 1.84030 & -0.60934 \\ \mathrm{C} & -1.64511 & 3.22364 & -0.18253 \\ \mathrm{H} & -2.02268 & 1.60410 & -1.29643 \\ \mathrm{O} & -1.45334 & -1.85446 & 0.30661 \\ \mathrm{C} & -1.96479 & -3.15265 & -0.15761 \\ \mathrm{H} & -1.87276 & -1.64723 & 1.15402 \\ \mathrm{O} & 2.81230 & -0.18350 & -0.61610 \\ \mathrm{H} & 3.34651 & 0.35077 & -1.22311 \\ \mathrm{H} & 3.44971 & -0.58295 & -0.00547 \\ \mathrm{O} & -1.07430 & 0.28557 & 2.05721 \\ \mathrm{C} & -2.30745 & 0.93981 & 2.54092 \\ \mathrm{H} & -0.64306 & -0.13079 & 2.81721 \\ \mathrm{O} & 1.36478 & -1.30384 & 1.63382 \\ \mathrm{C} & 1.45843 & -2.73530 & 1.98552 \\ \mathrm{H} & 1.94720 & -0.81207 & 2.23083 \\ \mathrm{O} & 1.21376 & 1.78238 & 1.08860 \\ \mathrm{C} & 2.30799 & 2.76046 & 0.94841 \\ \mathrm{H} & 0.72367 & 1.99005 & 1.89675 \\ \mathrm{O} & -1.59261 & -0.42376 & -2.20852 \\ \mathrm{H} & -2.40794 & -0.94555 & -2.15482 \\ \mathrm{H} & -1.48408 & -0.21524 & -3.14946 \\ \mathrm{O} & 0.96835 & -1.94006 & -1.71915 \\ \mathrm{H} & 0.49366 & -2.54145 & -2.31202 \\ \mathrm{H} & 1.90660 & -2.17290 & -1.79137 \\ \mathrm{O} & 0.96066 & 1.18963 & -2.35898 \\ \mathrm{H} & 1.43652 & 0.85274 & -3.13406 \\ \mathrm{H} & 0.74158 & 2.10984 & -2.57218 \\ \mathrm{H} & -0.93460 & 3.45395 & 0.61316 \\ \mathrm{H} & -2.67105 & 3.30425 & 0.18995 \\ \mathrm{H} & -1.49595 & 3.90320 & -1.02785 \\ \mathrm{H} & -1.48588 & -3.36685 & -1.11565 \\ \mathrm{H} & -3.05142 & -3.09613 & -0.28251 \\ \mathrm{H} & -1.70785 & -3.93179 & 0.56722 \\ \mathrm{H} & -2.89872 & 1.18712 & 1.65738 \\ \mathrm{H} & -2.05401 & 1.84515 & 3.10196 \\ \mathrm{H} & -2.86312 & 0.24615 & 3.18013 \\ \mathrm{H} & 2.73306 & 2.62193 & -0.04748 \\ \mathrm{H} & 3.06551 & 2.57643 & 1.71681 \\ \mathrm{H} & 1.90567 & 3.77410 & 1.04238 \\ \mathrm{H} & 0.85854 & -3.27597 & 1.25102 \\ \mathrm{H} & 1.06306 & -2.89252 & 2.99418 \\ \mathrm{H} & 2.50259 & -3.05964 & 1.93154 \\ & & & \end{array}$




$\begin{array}{lrrr}\mathrm{Cm} & 0.22129 & 0.00708 & -0.19586 \\ \mathrm{O} & -0.52340 & 2.41701 & -0.06288 \\ \mathrm{C} & -0.06572 & 3.74342 & 0.38378 \\ \mathrm{H} & -1.46347 & 2.47960 & -0.28316 \\ \mathrm{O} & -1.22943 & -1.78231 & 0.77784 \\ \mathrm{C} & -1.87567 & -3.06062 & 0.44444 \\ \mathrm{H} & -1.47501 & -1.54603 & 1.68291 \\ \mathrm{O} & 2.48768 & -0.37548 & -1.47244 \\ \mathrm{H} & 2.78779 & 0.06546 & -2.28199 \\ \mathrm{H} & 3.20135 & -0.98353 & -1.22591 \\ \mathrm{O} & -0.32013 & 0.39699 & 2.27470 \\ \mathrm{C} & -1.19523 & 1.28206 & 3.06726 \\ \mathrm{H} & 0.24686 & -0.08804 & 2.89192 \\ \mathrm{O} & 1.76467 & -1.48377 & 1.13301 \\ \mathrm{C} & 1.84628 & -2.91709 & 1.47168 \\ \mathrm{H} & 2.52810 & -1.03520 & 1.52448 \\ \mathrm{O} & 2.19241 & 1.34745 & 0.80660 \\ \mathrm{H} & 3.02810 & 1.52573 & 0.34819 \\ \mathrm{H} & 2.18157 & 1.94266 & 1.57178 \\ \mathrm{O} & -2.11637 & 0.36665 & -1.14926 \\ \mathrm{C} & -3.51482 & 0.05702 & -0.79993 \\ \mathrm{H} & -2.10104 & 0.69916 & -2.05865 \\ \mathrm{O} & 0.00931 & -1.76745 & -2.00373 \\ \mathrm{H} & -0.79004 & -2.18300 & -2.36126 \\ \mathrm{H} & 0.75400 & -2.20458 & -2.44428 \\ \mathrm{O} & 0.30773 & 1.19772 & -2.54091 \\ \mathrm{H} & 0.38625 & 0.82579 & -3.43315 \\ \mathrm{H} & 0.31528 & 2.16042 & -2.65703 \\ \mathrm{H} & 1.01925 & 3.76928 & 0.26132 \\ \mathrm{H} & -0.34497 & 3.90144 & 1.43089 \\ \mathrm{H} & -0.52023 & 4.51385 & -0.24700 \\ \mathrm{H} & -1.32389 & -3.49645 & -0.39123 \\ \mathrm{H} & -2.92172 & -2.88849 & 0.17168 \\ \mathrm{H} & -1.81616 & -3.73209 & 1.30672 \\ \mathrm{H} & -1.88673 & 1.75318 & 2.36580 \\ \mathrm{H} & -0.59201 & 2.03899 & 3.57865 \\ \mathrm{H} & -1.75207 & 0.68636 & 3.79749 \\ \mathrm{H} & 1.04567 & -3.41584 & 0.92319 \\ \mathrm{H} & 1.71124 & -3.04939 & 2.55003 \\ \mathrm{H} & 2.81854 & -3.31156 & 1.15965 \\ \mathrm{H} & -3.52107 & -0.23271 & 0.25261 \\ \mathrm{H} & -3.88112 & -0.76270 & -1.42629 \\ \mathrm{H} & -4.13257 & 0.94874 & -0.94590\end{array}$




\begin{tabular}{|c|c|c|c|}
\hline $\mathrm{Cm}$ & 0.19193 & -0.08940 & -0.03637 \\
\hline 0 & 0.44939 & -2.48384 & 0.68044 \\
\hline C & 0.65850 & -3.82062 & 0.09595 \\
\hline $\mathrm{H}$ & 0.31784 & -2.59243 & 1.63309 \\
\hline O & -1.86249 & 0.70431 & 1.12707 \\
\hline C & -2.36230 & 1.72334 & 2.06101 \\
\hline $\mathrm{H}$ & -2.61355 & 0.19494 & 0.78848 \\
\hline O & 2.00533 & 0.92272 & -1.65832 \\
\hline $\mathrm{H}$ & 2.96022 & 0.78387 & -1.56875 \\
\hline $\mathrm{H}$ & 1.91123 & 1.64928 & -2.29259 \\
\hline 0 & -2.04784 & -1.29459 & -0.73561 \\
\hline C & -2.78221 & -2.52025 & -0.37022 \\
\hline $\mathrm{H}$ & -2.47203 & -0.92404 & -1.52262 \\
\hline O & -0.91144 & 1.29732 & -1.81320 \\
\hline C & -1.75065 & 2.51127 & -1.81370 \\
\hline $\mathrm{H}$ & -0.79954 & 1.00988 & -2.73103 \\
\hline 0 & 0.51278 & -1.33781 & -2.23246 \\
\hline $\mathrm{H}$ & 1.26462 & -1.18389 & -2.82520 \\
\hline $\mathrm{H}$ & 0.03197 & -2.09798 & -2.59304 \\
\hline O & 0.36879 & -0.36047 & 2.59803 \\
\hline $\mathrm{H}$ & -0.36590 & -0.27200 & 3.22477 \\
\hline $\mathrm{H}$ & 1.14914 & -0.56472 & 3.13691 \\
\hline O & 0.91646 & 2.04805 & 0.99876 \\
\hline $\mathrm{H}$ & 0.99945 & 2.01653 & 1.96244 \\
\hline $\mathrm{C}$ & 1.26097 & 3.40925 & 0.55359 \\
\hline O & 2.65101 & -0.56154 & 0.69291 \\
\hline $\mathrm{H}$ & 3.03798 & -1.44748 & 0.76627 \\
\hline $\mathrm{H}$ & 1.06228 & -3.67092 & -0.90744 \\
\hline $\mathrm{H}$ & -0.29214 & -4.36177 & 0.05672 \\
\hline $\mathrm{H}$ & 1.38251 & -4.37211 & 0.70413 \\
\hline $\mathrm{H}$ & -1.50405 & 2.32165 & 2.37382 \\
\hline $\mathrm{H}$ & -2.82486 & 1.23550 & 2.92579 \\
\hline $\mathrm{H}$ & -3.09473 & 2.35997 & 1.55428 \\
\hline $\mathrm{H}$ & -2.40272 & -2.83746 & 0.60355 \\
\hline $\mathrm{H}$ & -2.61109 & -3.29797 & -1.12180 \\
\hline $\mathrm{H}$ & -3.85179 & -2.29853 & -0.29714 \\
\hline $\mathrm{H}$ & -1.79594 & 2.85989 & -0.78036 \\
\hline $\mathrm{H}$ & -2.75295 & 2.26530 & -2.17857 \\
\hline $\mathrm{H}$ & -1.29079 & 3.27295 & -2.45114 \\
\hline $\mathrm{H}$ & 1.04504 & 3.45792 & -0.51547 \\
\hline $\mathrm{H}$ & 2.32305 & 3.59931 & 0.73744 \\
\hline $\mathrm{H}$ & 0.64345 & 4.13589 & 1.0909 \\
\hline $\mathrm{H}$ & 3.35013 & 0.05067 & 0.9698 \\
\hline
\end{tabular}




\begin{tabular}{|c|c|c|c|}
\hline $\mathrm{Cm}$ & 0.15038 & 0.03086 & 0.08219 \\
\hline 0 & -1.07361 & 2.04524 & -0.84307 \\
\hline $\mathrm{C}$ & -2.01427 & 3.09778 & -0.41981 \\
\hline $\mathrm{H}$ & -0.96066 & 2.11053 & -1.80258 \\
\hline O & -0.45555 & -1.83738 & -1.47351 \\
\hline $\mathrm{C}$ & 0.10560 & -2.81561 & -2.41601 \\
\hline $\mathrm{H}$ & -1.39806 & -2.02707 & -1.35989 \\
\hline 0 & 1.77165 & 0.13199 & 2.18055 \\
\hline $\mathrm{H}$ & 2.41628 & 0.82359 & 2.39394 \\
\hline $\mathrm{H}$ & 1.84852 & -0.51887 & 2.89443 \\
\hline O & -2.38419 & -0.60204 & 0.08356 \\
\hline $\mathrm{C}$ & -3.61718 & -0.18789 & -0.61252 \\
\hline $\mathrm{H}$ & -2.63413 & -1.23459 & 0.77212 \\
\hline O & -0.23641 & -1.90099 & 1.67393 \\
\hline $\mathrm{C}$ & 0.03493 & -3.35005 & 1.60129 \\
\hline $\mathrm{H}$ & -0.61573 & -1.71080 & 2.54417 \\
\hline O & -0.96057 & 1.02517 & 2.14054 \\
\hline $\mathrm{H}$ & -0.48701 & 1.39722 & 2.90052 \\
\hline $\mathrm{H}$ & -1.89771 & 1.22590 & 2.28510 \\
\hline O & 0.78172 & 0.56325 & -2.39587 \\
\hline $\mathrm{H}$ & 0.51002 & 0.07415 & -3.18759 \\
\hline $\mathrm{H}$ & 1.45427 & 1.19523 & -2.69352 \\
\hline O & 2.35914 & -1.10322 & -0.35377 \\
\hline $\mathrm{H}$ & 2.78003 & -1.34651 & -1.19197 \\
\hline $\mathrm{H}$ & 2.98778 & -1.35391 & 0.33983 \\
\hline O & 1.64093 & 2.06815 & 0.01828 \\
\hline C & 3.05486 & 2.43832 & 0.18714 \\
\hline $\mathrm{H}$ & 1.13180 & 2.87827 & -0.12717 \\
\hline $\mathrm{H}$ & -1.94802 & 3.16615 & 0.66813 \\
\hline $\mathrm{H}$ & -3.03044 & 2.83700 & -0.73115 \\
\hline $\mathrm{H}$ & -1.71543 & 4.05170 & -0.86609 \\
\hline $\mathrm{H}$ & 1.15204 & -2.54740 & -2.58000 \\
\hline $\mathrm{H}$ & -0.44440 & -2.77095 & -3.36209 \\
\hline $\mathrm{H}$ & 0.04257 & -3.82175 & -1.98906 \\
\hline $\mathrm{H}$ & -3.30753 & 0.37749 & -1.49402 \\
\hline $\mathrm{H}$ & -4.22904 & 0.43356 & 0.04942 \\
\hline $\mathrm{H}$ & -4.17975 & -1.07540 & -0.91963 \\
\hline $\mathrm{H}$ & 0.55175 & -3.52322 & 0.65538 \\
\hline $\mathrm{H}$ & -0.90913 & -3.90367 & 1.62957 \\
\hline $\mathrm{H}$ & 0.67403 & -3.64625 & 2.43924 \\
\hline $\mathrm{H}$ & 3.62746 & 1.50780 & 0.2108 \\
\hline $\mathrm{H}$ & 3.18372 & 3.00294 & 1.11786 \\
\hline $\mathrm{H}$ & 3.38055 & 3.04671 & -0.66287 \\
\hline
\end{tabular}




\begin{tabular}{|c|c|c|c|}
\hline $\mathrm{Cm}$ & 0.10898 & -0.04556 & 0.16383 \\
\hline 0 & -1.80176 & -1.29626 & -0.85118 \\
\hline $\mathrm{C}$ & -2.15790 & -2.50819 & -1.60435 \\
\hline $\mathrm{H}$ & -2.61104 & -0.80202 & -0.65143 \\
\hline O & -0.00030 & 2.09845 & -1.08541 \\
\hline $\mathrm{C}$ & -0.00641 & 3.55196 & -0.85296 \\
\hline $\mathrm{H}$ & 0.00074 & 1.94865 & -2.04147 \\
\hline 0 & 1.77112 & -0.68543 & 2.10698 \\
\hline $\mathrm{H}$ & 1.66158 & -1.26412 & 2.87661 \\
\hline $\mathrm{H}$ & 2.72719 & -0.55333 & 2.02163 \\
\hline O & 0.41842 & -0.32076 & -2.50309 \\
\hline $\mathrm{H}$ & -0.27037 & -0.61741 & -3.11835 \\
\hline $\mathrm{H}$ & 1.19178 & -0.13122 & -3.05759 \\
\hline O & 2.51979 & 0.45107 & -0.40400 \\
\hline $\mathrm{C}$ & 3.32350 & 1.68174 & -0.53138 \\
\hline $\mathrm{H}$ & 3.07992 & -0.29977 & -0.64781 \\
\hline O & 1.10711 & -2.25112 & -0.44628 \\
\hline $\mathrm{C}$ & 1.48751 & -3.50355 & 0.23201 \\
\hline $\mathrm{H}$ & 1.18110 & -2.39155 & -1.40099 \\
\hline O & -2.28045 & 0.97760 & 0.61078 \\
\hline $\mathrm{C}$ & -3.15459 & 2.01918 & 0.04043 \\
\hline $\mathrm{H}$ & -2.66240 & 0.70448 & 1.45709 \\
\hline O & 0.26210 & 1.64631 & 2.02467 \\
\hline $\mathrm{H}$ & -0.31445 & 2.39195 & 2.24912 \\
\hline $\mathrm{H}$ & 0.94900 & 1.61669 & 2.70831 \\
\hline O & -1.09881 & -1.28474 & 2.09444 \\
\hline $\mathrm{H}$ & -1.09613 & -1.12406 & 3.05106 \\
\hline $\mathrm{H}$ & -1.64540 & -2.07639 & 1.97055 \\
\hline $\mathrm{H}$ & -1.23158 & -3.05702 & -1.78697 \\
\hline $\mathrm{H}$ & -2.63108 & -2.22822 & -2.55183 \\
\hline $\mathrm{H}$ & -2.84202 & -3.12338 & -1.01079 \\
\hline $\mathrm{H}$ & 0.18200 & 3.70881 & 0.21081 \\
\hline $\mathrm{H}$ & -0.97814 & 3.96679 & -1.13684 \\
\hline $\mathrm{H}$ & 0.79126 & 4.01679 & -1.44071 \\
\hline $\mathrm{H}$ & 2.72785 & 2.49160 & -0.10592 \\
\hline $\mathrm{H}$ & 3.53880 & 1.87643 & -1.58699 \\
\hline $\mathrm{H}$ & 4.25551 & 1.56856 & 0.03139 \\
\hline $\mathrm{H}$ & 1.28709 & -3.36166 & 1.29557 \\
\hline $\mathrm{H}$ & 2.55190 & -3.70027 & 0.06858 \\
\hline $\mathrm{H}$ & 0.88158 & -4.32871 & -0.15569 \\
\hline $\mathrm{H}$ & -2.84003 & 2.16295 & -0.99538 \\
\hline $\mathrm{H}$ & -3.04974 & 2.94989 & 0.60770 \\
\hline $\mathrm{H}$ & -4.19447 & 1.67832 & 0.06855 \\
\hline
\end{tabular}




\begin{tabular}{|c|c|c|c|}
\hline $\mathrm{Cm}$ & -0.06139 & -0.07103 & 0.06925 \\
\hline 0 & -0.99014 & -1.74351 & -1.56356 \\
\hline $\mathrm{C}$ & -0.75115 & -3.08980 & -2.10797 \\
\hline $\mathrm{H}$ & -1.79245 & -1.38500 & -1.97182 \\
\hline O & 0.12581 & 2.00872 & -1.34346 \\
\hline $\mathrm{H}$ & 0.08353 & 2.84020 & -0.85086 \\
\hline $\mathrm{C}$ & 0.44064 & 2.34193 & -2.74475 \\
\hline O & 0.34968 & -0.21804 & 2.71656 \\
\hline $\mathrm{H}$ & -0.09731 & -0.75850 & 3.38602 \\
\hline $\mathrm{H}$ & 1.21596 & -0.00853 & 3.09753 \\
\hline O & 1.65674 & -0.57493 & -1.82742 \\
\hline $\mathrm{H}$ & 1.45734 & -1.06130 & -2.64204 \\
\hline $\mathrm{H}$ & 2.47030 & -0.08198 & -2.01485 \\
\hline O & 2.19564 & 0.84302 & 0.76644 \\
\hline $\mathrm{C}$ & 2.75745 & 2.19588 & 0.93459 \\
\hline $\mathrm{H}$ & 2.91614 & 0.20671 & 0.87985 \\
\hline O & 1.29835 & -2.11380 & 0.51762 \\
\hline $\mathrm{C}$ & 1.35775 & -3.19864 & 1.51205 \\
\hline $\mathrm{H}$ & 1.94298 & -2.30203 & -0.17966 \\
\hline O & -2.38975 & 0.55857 & -1.04510 \\
\hline $\mathrm{H}$ & -2.52239 & 1.27671 & -1.68235 \\
\hline $\mathrm{H}$ & -3.27301 & 0.35346 & -0.70122 \\
\hline O & -0.93661 & 1.82521 & 1.43102 \\
\hline C & -1.83866 & 2.98653 & 1.36624 \\
\hline $\mathrm{H}$ & -0.65181 & 1.70747 & 2.35020 \\
\hline O & -1.96938 & -1.31513 & 1.26710 \\
\hline $\mathrm{H}$ & -2.54803 & -1.03450 & 1.99307 \\
\hline $\mathrm{H}$ & -2.28995 & -2.19329 & 1.00908 \\
\hline $\mathrm{H}$ & 0.14286 & -3.47982 & -1.61683 \\
\hline $\mathrm{H}$ & -0.59931 & -3.02873 & -3.19117 \\
\hline $\mathrm{H}$ & -1.60810 & -3.73419 & -1.8861 \\
\hline $\mathrm{H}$ & 1.91200 & 2.87792 & 1.05259 \\
\hline $\mathrm{H}$ & 3.35183 & 2.46497 & 0.05542 \\
\hline $\mathrm{H}$ & 3.37835 & 2.22365 & 1.83555 \\
\hline $\mathrm{H}$ & 0.49911 & -3.07098 & 2.17450 \\
\hline $\mathrm{H}$ & 2.29233 & -3.12954 & 2.07797 \\
\hline $\mathrm{H}$ & 1.29150 & -4.16527 & 1.0027 \\
\hline $\mathrm{H}$ & -2.16152 & 3.08486 & 0.3272 \\
\hline $\mathrm{H}$ & -1.30090 & 3.88538 & 1.68583 \\
\hline $\mathrm{H}$ & -2.70710 & 2.81530 & 2.01045 \\
\hline $\mathrm{H}$ & 0.28093 & 1.43552 & -3.3329 \\
\hline $\mathrm{H}$ & 1.48088 & 2.67510 & -2.8232 \\
\hline $\mathrm{H}$ & -0.23482 & 3.13120 & -3.09020 \\
\hline
\end{tabular}




\begin{tabular}{|c|c|c|c|}
\hline $\mathrm{Cm}$ & 0.17399 & -0.09040 & -0.19601 \\
\hline 0 & -2.31075 & 0.34637 & -0.31465 \\
\hline $\mathrm{C}$ & -3.31370 & 1.40512 & -0.12095 \\
\hline $\mathrm{H}$ & -2.77299 & -0.49730 & -0.42142 \\
\hline O & 1.12397 & -2.04049 & 1.13906 \\
\hline $\mathrm{H}$ & 1.73211 & -2.71880 & 0.80951 \\
\hline $\mathrm{H}$ & 0.97010 & -2.25306 & 2.07141 \\
\hline O & 1.88634 & 1.19817 & -1.65046 \\
\hline $\mathrm{H}$ & 1.78216 & 1.53479 & -2.55353 \\
\hline $\mathrm{H}$ & 2.71245 & 1.58324 & -1.32127 \\
\hline O & -0.58179 & 0.06115 & 2.20673 \\
\hline C & -1.78775 & -0.23257 & 3.00360 \\
\hline $\mathrm{H}$ & 0.12362 & 0.32302 & 2.81625 \\
\hline O & 2.10037 & 0.81363 & 1.20860 \\
\hline $\mathrm{C}$ & 3.37331 & 0.36093 & 1.80189 \\
\hline $\mathrm{H}$ & 2.01886 & 1.76669 & 1.35880 \\
\hline O & -0.16621 & 2.36656 & 0.33320 \\
\hline $\mathrm{C}$ & -0.03720 & 3.67691 & -0.33286 \\
\hline $\mathrm{H}$ & -0.70421 & 2.49471 & 1.12791 \\
\hline O & -1.23170 & -2.16992 & -0.77760 \\
\hline $\mathrm{C}$ & -1.62273 & -3.42278 & -0.10360 \\
\hline $\mathrm{H}$ & -1.45453 & -2.25561 & -1.71597 \\
\hline O & 1.66764 & -1.63587 & -1.64704 \\
\hline $\mathrm{H}$ & 1.54631 & -2.55477 & -1.93100 \\
\hline $\mathrm{H}$ & 2.48432 & -1.33638 & -2.07528 \\
\hline O & -0.63687 & 0.10910 & -2.64456 \\
\hline $\mathrm{H}$ & -0.22361 & -0.16536 & -3.47782 \\
\hline $\mathrm{H}$ & -1.46865 & 0.54090 & -2.89368 \\
\hline $\mathrm{H}$ & -2.81352 & 2.35871 & -0.30054 \\
\hline $\mathrm{H}$ & -3.71145 & 1.36251 & 0.89854 \\
\hline $\mathrm{H}$ & -4.12275 & 1.27546 & -0.84679 \\
\hline $\mathrm{H}$ & -2.54530 & -0.59623 & 2.30704 \\
\hline $\mathrm{H}$ & -2.13187 & 0.68090 & 3.49924 \\
\hline $\mathrm{H}$ & -1.55884 & -1.00335 & 3.74634 \\
\hline $\mathrm{H}$ & 3.46871 & -0.70145 & 1.57131 \\
\hline $\mathrm{H}$ & 3.34919 & 0.51405 & 2.88585 \\
\hline $\mathrm{H}$ & 4.20519 & 0.91903 & 1.35971 \\
\hline $\mathrm{H}$ & 0.61517 & 3.52989 & -1.1950 \\
\hline $\mathrm{H}$ & 0.41046 & 4.39488 & 0.36185 \\
\hline $\mathrm{H}$ & -1.02168 & 4.02726 & -0.66013 \\
\hline $\mathrm{H}$ & -1.50580 & -3.25490 & 0.96902 \\
\hline $\mathrm{H}$ & -0.97666 & -4.24261 & -0.43400 \\
\hline $\mathrm{H}$ & -2.66884 & -3.64845 & -0.33422 \\
\hline
\end{tabular}




\begin{tabular}{|c|c|c|c|}
\hline $\mathrm{Cm}$ & -0.14312 & 0.09847 & 0.04066 \\
\hline 0 & -1.61992 & -1.91303 & -0.32839 \\
\hline $\mathrm{C}$ & -1.71623 & -3.34219 & 0.00920 \\
\hline $\mathrm{H}$ & -2.33358 & -1.69910 & -0.94676 \\
\hline O & 0.34260 & 1.38471 & -2.13530 \\
\hline $\mathrm{H}$ & 0.26288 & 2.33356 & -2.31590 \\
\hline $\mathrm{H}$ & 0.79117 & 1.00910 & -2.90738 \\
\hline O & 0.49007 & 0.91614 & 2.46272 \\
\hline $\mathrm{H}$ & -0.06522 & 0.82999 & 3.25301 \\
\hline $\mathrm{H}$ & 1.38206 & 1.10354 & 2.79283 \\
\hline O & 1.09399 & -1.34458 & -1.68303 \\
\hline C & 0.76057 & -2.34325 & -2.71792 \\
\hline $\mathrm{H}$ & 2.03882 & -1.14659 & -1.75761 \\
\hline O & 2.31988 & 0.64480 & 0.16872 \\
\hline C & 3.19024 & 1.77290 & -0.21492 \\
\hline $\mathrm{H}$ & 2.85747 & -0.00005 & 0.65077 \\
\hline O & 1.02609 & -1.80973 & 1.19580 \\
\hline C & 1.22919 & -2.37452 & 2.54282 \\
\hline $\mathrm{H}$ & 1.34093 & -2.45935 & 0.55056 \\
\hline 0 & -2.30365 & 0.56719 & -1.37065 \\
\hline $\mathrm{H}$ & -2.31387 & 0.82715 & -2.30474 \\
\hline $\mathrm{H}$ & -3.19362 & 0.75977 & -1.03677 \\
\hline O & -0.55915 & 2.53197 & 0.46054 \\
\hline C & -1.15808 & 3.71281 & -0.17934 \\
\hline $\mathrm{H}$ & -0.17440 & 2.80544 & 1.30613 \\
\hline O & -2.16469 & 0.15967 & 1.64174 \\
\hline $\mathrm{H}$ & -2.59200 & 0.91716 & 2.07067 \\
\hline $\mathrm{H}$ & -2.71340 & -0.60932 & 1.86006 \\
\hline $\mathrm{H}$ & -1.03750 & -3.51878 & 0.84532 \\
\hline $\mathrm{H}$ & -1.42822 & -3.94989 & -0.85498 \\
\hline $\mathrm{H}$ & -2.74225 & -3.57683 & 0.31013 \\
\hline $\mathrm{H}$ & -0.32298 & -2.47099 & -2.69441 \\
\hline $\mathrm{H}$ & 1.26336 & -3.28935 & -2.49237 \\
\hline $\mathrm{H}$ & 1.07326 & -1.97436 & -3.70008 \\
\hline $\mathrm{H}$ & 2.54964 & 2.51373 & -0.69693 \\
\hline $\mathrm{H}$ & 3.95900 & 1.42399 & -0.9119 \\
\hline $\mathrm{H}$ & 3.65406 & 2.20069 & 0.67982 \\
\hline $\mathrm{H}$ & 0.75389 & -1.69214 & 3.24937 \\
\hline $\mathrm{H}$ & 2.30158 & -2.45273 & 2.7494 \\
\hline $\mathrm{H}$ & 0.75876 & -3.36123 & 2.60389 \\
\hline $\mathrm{H}$ & -1.69799 & 3.35980 & -1.0615 \\
\hline $\mathrm{H}$ & -0.37118 & 4.42127 & -0.4611 \\
\hline $\mathrm{H}$ & -1.86150 & 4.18868 & 0.5113 \\
\hline
\end{tabular}




\begin{tabular}{|c|c|c|c|}
\hline $\mathrm{Cm}$ & 0.07012 & 0.03826 & 0.15924 \\
\hline 0 & -1.09604 & -2.14437 & 0.71132 \\
\hline $\mathrm{C}$ & -0.85802 & -3.46510 & 1.31748 \\
\hline $\mathrm{H}$ & -1.96167 & -2.16314 & 0.27922 \\
\hline O & -0.01125 & 1.20987 & -2.10819 \\
\hline $\mathrm{H}$ & -0.25661 & 2.12345 & -2.31648 \\
\hline $\mathrm{H}$ & 0.18872 & 0.79255 & -2.95870 \\
\hline 0 & 1.22327 & 1.00370 & 2.32526 \\
\hline $\mathrm{H}$ & 0.85004 & 1.00802 & 3.22022 \\
\hline $\mathrm{H}$ & 2.12451 & 1.34981 & 2.41693 \\
\hline O & 0.81331 & -1.50935 & -1.69183 \\
\hline $\mathrm{C}$ & 0.28219 & -2.69189 & -2.39512 \\
\hline $\mathrm{H}$ & 1.69165 & -1.31964 & -2.05241 \\
\hline O & 2.42688 & 0.72184 & -0.38442 \\
\hline $\mathrm{C}$ & 3.11880 & 1.82852 & -1.07016 \\
\hline $\mathrm{H}$ & 3.09492 & 0.12021 & -0.02476 \\
\hline O & 1.84538 & -1.57580 & 1.16727 \\
\hline $\mathrm{H}$ & 2.18824 & -1.55471 & 2.07399 \\
\hline $\mathrm{H}$ & 2.07643 & -2.45221 & 0.82263 \\
\hline O & -2.33907 & -0.00270 & -0.63883 \\
\hline $\mathrm{C}$ & -3.05010 & -0.05995 & -1.92891 \\
\hline $\mathrm{H}$ & -2.97778 & 0.24296 & 0.04624 \\
\hline O & -0.43963 & 2.47513 & 0.49890 \\
\hline C & -1.30665 & 3.51026 & -0.08511 \\
\hline $\mathrm{H}$ & 0.11782 & 2.89203 & 1.17192 \\
\hline O & -1.58031 & 0.26609 & 2.18086 \\
\hline $\mathrm{H}$ & -1.92127 & 1.06115 & 2.61882 \\
\hline $\mathrm{H}$ & -1.94930 & -0.48514 & 2.67056 \\
\hline $\mathrm{H}$ & -0.05479 & -3.34466 & 2.04718 \\
\hline $\mathrm{H}$ & -0.57472 & -4.18597 & 0.54283 \\
\hline $\mathrm{H}$ & -1.76697 & -3.79850 & 1.82802 \\
\hline $\mathrm{H}$ & -0.72665 & -2.85952 & -2.01300 \\
\hline $\mathrm{H}$ & 0.91623 & -3.55982 & -2.18769 \\
\hline $\mathrm{H}$ & 0.24953 & -2.49458 & -3.47132 \\
\hline $\mathrm{H}$ & 2.34690 & 2.52400 & -1.4041 \\
\hline $\mathrm{H}$ & 3.67584 & 1.43898 & -1.92833 \\
\hline $\mathrm{H}$ & 3.79811 & 2.32509 & -0.36978 \\
\hline $\mathrm{H}$ & -2.35204 & -0.47758 & -2.6574 \\
\hline $\mathrm{H}$ & -3.35922 & 0.94601 & -2.23020 \\
\hline $\mathrm{H}$ & -3.92354 & -0.71254 & -1.83239 \\
\hline $\mathrm{H}$ & -2.01146 & 2.99843 & $-0.7457^{\prime}$ \\
\hline $\mathrm{H}$ & -0.70057 & 4.23243 & -0.6439 \\
\hline $\mathrm{H}$ & -1.85738 & 4.02226 & 0.71039 \\
\hline
\end{tabular}




\begin{tabular}{|c|c|c|c|}
\hline $\mathrm{Cm}$ & 0.17271 & -0.06454 & -0.00213 \\
\hline 0 & -1.80923 & 1.48724 & 0.51963 \\
\hline $\mathrm{C}$ & -2.76252 & 1.77607 & 1.60509 \\
\hline $\mathrm{H}$ & -2.16567 & 1.85687 & -0.30059 \\
\hline O & 0.16503 & -1.56962 & -2.05675 \\
\hline $\mathrm{H}$ & 0.85487 & -1.63732 & -2.73363 \\
\hline $\mathrm{H}$ & -0.52031 & -2.20582 & -2.30914 \\
\hline 0 & 2.30854 & -0.03235 & 1.49373 \\
\hline $\mathrm{H}$ & 2.80573 & 0.73245 & 1.82156 \\
\hline $\mathrm{H}$ & 2.78450 & -0.81106 & 1.81965 \\
\hline O & -1.87340 & -1.53048 & -0.01122 \\
\hline $\mathrm{C}$ & -3.31182 & -1.43967 & -0.31697 \\
\hline $\mathrm{H}$ & -1.68179 & -2.44085 & 0.25646 \\
\hline O & 0.82013 & -2.40818 & 0.78607 \\
\hline $\mathrm{C}$ & 1.48451 & -3.61481 & 0.25651 \\
\hline $\mathrm{H}$ & 0.63123 & -2.55837 & 1.72381 \\
\hline O & -0.40362 & -0.40994 & 2.48241 \\
\hline $\mathrm{H}$ & 0.15904 & -0.14586 & 3.22694 \\
\hline $\mathrm{H}$ & -1.27079 & -0.61010 & 2.86734 \\
\hline O & -0.48693 & 1.38847 & -1.98352 \\
\hline $\mathrm{C}$ & -1.11913 & 1.22438 & -3.30726 \\
\hline $\mathrm{H}$ & -0.13282 & 2.28819 & -1.92985 \\
\hline O & 2.30603 & 0.20833 & -1.41452 \\
\hline $\mathrm{H}$ & 2.41077 & 0.64362 & -2.27437 \\
\hline $\mathrm{H}$ & 3.20503 & -0.00388 & -1.12037 \\
\hline O & 0.84608 & 2.32230 & 0.49325 \\
\hline C & 2.04049 & 3.17461 & 0.59516 \\
\hline $\mathrm{H}$ & 0.08844 & 2.80985 & 0.84841 \\
\hline $\mathrm{H}$ & -2.21195 & 1.70053 & 2.54569 \\
\hline $\mathrm{H}$ & -3.59037 & 1.05970 & 1.5778 \\
\hline $\mathrm{H}$ & -3.14318 & 2.79587 & 1.49019 \\
\hline $\mathrm{H}$ & -3.48943 & -0.44067 & -0.72014 \\
\hline $\mathrm{H}$ & -3.89391 & -1.59345 & 0.59741 \\
\hline $\mathrm{H}$ & -3.57604 & -2.19426 & -1.06461 \\
\hline $\mathrm{H}$ & 1.74808 & -3.40055 & -0.78065 \\
\hline $\mathrm{H}$ & 0.79713 & -4.46574 & 0.30617 \\
\hline $\mathrm{H}$ & 2.38736 & -3.82372 & 0.83975 \\
\hline $\mathrm{H}$ & -1.61097 & 0.24988 & -3.30369 \\
\hline $\mathrm{H}$ & -0.35602 & 1.26382 & -4.09133 \\
\hline $\mathrm{H}$ & -1.85806 & 2.01808 & -3.45750 \\
\hline $\mathrm{H}$ & 2.84578 & 2.65978 & 0.06516 \\
\hline $\mathrm{H}$ & 2.29983 & 3.32974 & 1.64884 \\
\hline $\mathrm{H}$ & 1.84575 & 4.13894 & 0.11504 \\
\hline
\end{tabular}




\begin{tabular}{|c|c|c|c|}
\hline $\mathrm{Cm}$ & -0.00547 & 0.01042 & 0.00084 \\
\hline 0 & -2.12328 & -1.40949 & -0.37080 \\
\hline $\mathrm{C}$ & -2.89002 & -2.47524 & 0.29731 \\
\hline $\mathrm{H}$ & -2.41121 & -1.36785 & -1.29398 \\
\hline O & 1.64308 & 0.03932 & -1.98838 \\
\hline $\mathrm{H}$ & 2.10927 & 0.77846 & -2.40738 \\
\hline $\mathrm{H}$ & 2.04687 & -0.76357 & -2.35006 \\
\hline O & 0.23296 & 1.41441 & 2.24378 \\
\hline $\mathrm{H}$ & -0.46815 & 1.89939 & 2.70582 \\
\hline $\mathrm{H}$ & 1.00754 & 1.46583 & 2.82461 \\
\hline O & 0.80815 & -2.32097 & -0.62564 \\
\hline $\mathrm{C}$ & 0.34408 & -3.49877 & -1.38101 \\
\hline $\mathrm{H}$ & 1.68036 & -2.52915 & -0.26113 \\
\hline O & 2.26737 & -0.35085 & 1.05784 \\
\hline $\mathrm{C}$ & 3.64575 & 0.11991 & 0.82264 \\
\hline $\mathrm{H}$ & 2.26759 & -0.89457 & 1.85902 \\
\hline O & -0.24719 & -1.45089 & 2.09114 \\
\hline $\mathrm{H}$ & -0.47092 & -1.14283 & 2.98290 \\
\hline $\mathrm{H}$ & -0.34057 & -2.41564 & 2.11468 \\
\hline O & -1.17067 & 0.47268 & -2.25216 \\
\hline $\mathrm{H}$ & -0.76943 & 0.33759 & -3.12464 \\
\hline $\mathrm{H}$ & -1.94666 & 1.03504 & -2.40134 \\
\hline O & 0.71851 & 2.35887 & -0.39208 \\
\hline C & 0.90463 & 3.30324 & -1.50507 \\
\hline $\mathrm{H}$ & 0.93827 & 2.81600 & 0.43246 \\
\hline O & -2.13334 & 1.22861 & 0.52435 \\
\hline C & -2.62253 & 2.57574 & 0.85438 \\
\hline $\mathrm{H}$ & -2.88686 & 0.62069 & 0.51256 \\
\hline $\mathrm{H}$ & -2.80960 & -2.30250 & 1.37312 \\
\hline $\mathrm{H}$ & -2.48239 & -3.45550 & 0.02857 \\
\hline $\mathrm{H}$ & -3.94039 & -2.40938 & -0.00378 \\
\hline $\mathrm{H}$ & -0.57630 & -3.20741 & -1.89091 \\
\hline $\mathrm{H}$ & 0.15585 & -4.32945 & -0.69291 \\
\hline $\mathrm{H}$ & 1.10182 & -3.78396 & -2.11781 \\
\hline $\mathrm{H}$ & 3.60462 & 0.79806 & -0.03179 \\
\hline $\mathrm{H}$ & 4.29395 & -0.73392 & 0.60026 \\
\hline $\mathrm{H}$ & 4.00870 & 0.65156 & 1.70826 \\
\hline $\mathrm{H}$ & 0.53813 & 2.80918 & -2.4081 \\
\hline $\mathrm{H}$ & 1.96634 & 3.55477 & -1.60213 \\
\hline $\mathrm{H}$ & 0.31775 & 4.20868 & -1.3211 \\
\hline $\mathrm{H}$ & -1.77238 & 3.25531 & 0.7599 \\
\hline $\mathrm{H}$ & -3.01893 & 2.58658 & 1.8759 \\
\hline $\mathrm{H}$ & -3.40602 & 2.86645 & 0.1471 \\
\hline
\end{tabular}




$\begin{array}{lrrr}\mathrm{Cm} & 0.21148 & -0.07181 & 0.04404 \\ \mathrm{O} & -1.27612 & 1.24344 & -1.51065 \\ \mathrm{C} & -1.52763 & 2.61153 & -1.99106 \\ \mathrm{H} & -1.95763 & 0.66072 & -1.87513 \\ \mathrm{O} & -0.68902 & -1.70453 & 1.83836 \\ \mathrm{H} & -0.43832 & -2.63543 & 1.93475 \\ \mathrm{H} & -1.44791 & -1.57860 & 2.42744 \\ \mathrm{O} & 2.84335 & -0.02967 & -0.14791 \\ \mathrm{H} & 3.36961 & 0.12432 & -0.94778 \\ \mathrm{H} & 3.45435 & 0.11609 & 0.59075 \\ \mathrm{O} & -1.41519 & 1.10691 & 1.57906 \\ \mathrm{C} & -2.78615 & 1.64896 & 1.51914 \\ \mathrm{H} & -1.09709 & 1.20126 & 2.48888 \\ \mathrm{O} & 1.31409 & 0.26360 & 2.36994 \\ \mathrm{H} & 1.31895 & -0.39871 & 3.07852 \\ \mathrm{H} & 1.83412 & 1.01132 & 2.70243 \\ \mathrm{O} & 0.94069 & 2.32576 & 0.22392 \\ \mathrm{C} & 2.00631 & 3.21459 & -0.27097 \\ \mathrm{H} & 0.30520 & 2.86823 & 0.71224 \\ \mathrm{O} & -1.59141 & -1.47802 & -1.11014 \\ \mathrm{C} & -2.87819 & -2.06615 & -0.69623 \\ \mathrm{H} & -1.32866 & -1.89477 & -1.94370 \\ \mathrm{O} & 1.32138 & -2.33423 & -0.14883 \\ \mathrm{C} & 0.98105 & -3.75969 & -0.26312 \\ \mathrm{H} & 2.28522 & -2.25189 & -0.10331 \\ \mathrm{O} & 0.95858 & -0.33604 & -2.39521 \\ \mathrm{H} & 1.51134 & -1.01577 & -2.81080 \\ \mathrm{H} & 0.68535 & 0.25773 & -3.11151 \\ \mathrm{H} & -0.65290 & 3.20742 & -1.72655 \\ \mathrm{H} & -2.42622 & 3.01514 & -1.51277 \\ \mathrm{H} & -1.65240 & 2.59810 & -3.07859 \\ \mathrm{H} & -3.14710 & 1.47842 & 0.50353 \\ \mathrm{H} & -2.76749 & 2.72058 & 1.74181 \\ \mathrm{H} & -3.42035 & 1.12295 & 2.23976 \\ \mathrm{H} & 2.58699 & 2.64517 & -0.99926 \\ \mathrm{H} & 2.64093 & 3.53091 & 0.56332 \\ \mathrm{H} & 1.55991 & 4.08774 & -0.75769 \\ \mathrm{H} & -3.24493 & -1.46828 & 0.14166 \\ \mathrm{H} & -2.73398 & -3.10694 & -0.38879 \\ \mathrm{H} & -3.58760 & -2.01162 & -1.52815 \\ \mathrm{H} & -0.10808 & -3.82332 & -0.32900 \\ \mathrm{H} & 1.34543 & -4.30055 & 0.61751 \\ \mathrm{H} & 1.43140 & -4.17700 & -1.16973\end{array}$




\begin{tabular}{|c|c|c|c|}
\hline $\mathrm{Cm}$ & 0.10104 & -0.12006 & 0.26084 \\
\hline 0 & -0.78751 & 0.60351 & -2.02945 \\
\hline $\mathrm{C}$ & -1.09304 & 1.82710 & -2.78941 \\
\hline $\mathrm{H}$ & -1.21688 & -0.14322 & -2.47068 \\
\hline O & -1.17097 & -1.68069 & 1.87933 \\
\hline $\mathrm{H}$ & -0.79507 & -2.50156 & 2.23154 \\
\hline $\mathrm{H}$ & -2.10394 & -1.68250 & 2.14110 \\
\hline 0 & 2.40082 & 0.37166 & 1.32989 \\
\hline $\mathrm{H}$ & 3.27682 & 0.42039 & 0.91793 \\
\hline $\mathrm{H}$ & 2.51687 & 0.71552 & 2.22844 \\
\hline O & -2.19587 & 0.81787 & 0.69206 \\
\hline $\mathrm{C}$ & -3.49595 & 0.94435 & 0.00882 \\
\hline $\mathrm{H}$ & -2.29950 & 1.14483 & 1.59770 \\
\hline O & -0.09651 & 0.83361 & 2.71221 \\
\hline $\mathrm{H}$ & -0.31588 & 0.30469 & 3.49520 \\
\hline $\mathrm{H}$ & 0.08881 & 1.72706 & 3.04080 \\
\hline O & 0.34387 & 2.36644 & 0.16359 \\
\hline $\mathrm{C}$ & 1.42814 & 3.34804 & -0.01684 \\
\hline $\mathrm{H}$ & -0.49619 & 2.84721 & 0.15534 \\
\hline O & -0.72983 & -2.06085 & -1.18958 \\
\hline $\mathrm{C}$ & -1.89048 & -2.97220 & -1.17034 \\
\hline $\mathrm{H}$ & -0.08974 & -2.40971 & -1.82708 \\
\hline O & 1.42797 & -2.25307 & 0.94395 \\
\hline $\mathrm{H}$ & 1.35691 & -3.16617 & 0.62674 \\
\hline $\mathrm{H}$ & 2.21940 & -2.23132 & 1.50315 \\
\hline O & 1.80494 & -0.31731 & -1.54767 \\
\hline C & 3.17710 & -0.79421 & -1.78367 \\
\hline $\mathrm{H}$ & 1.49338 & 0.13141 & -2.34740 \\
\hline $\mathrm{H}$ & -0.40518 & 2.59931 & -2.43986 \\
\hline $\mathrm{H}$ & -2.13158 & 2.12750 & -2.61553 \\
\hline $\mathrm{H}$ & -0.92664 & 1.64248 & -3.85553 \\
\hline $\mathrm{H}$ & -3.38271 & 0.48524 & -0.97495 \\
\hline $\mathrm{H}$ & -3.75709 & 2.00276 & -0.09127 \\
\hline $\mathrm{H}$ & -4.26467 & 0.41690 & 0.58233 \\
\hline $\mathrm{H}$ & 2.35997 & 2.78284 & -0.07379 \\
\hline $\mathrm{H}$ & 1.44991 & 4.03231 & 0.83744 \\
\hline $\mathrm{H}$ & 1.27071 & 3.90530 & -0.94610 \\
\hline $\mathrm{H}$ & -2.66461 & -2.48535 & -0.57310 \\
\hline $\mathrm{H}$ & -1.60680 & -3.92943 & -0.72107 \\
\hline $\mathrm{H}$ & -2.25008 & -3.12489 & -2.1930 \\
\hline $\mathrm{H}$ & 3.45253 & -1.41078 & -0.92461 \\
\hline $\mathrm{H}$ & 3.85409 & 0.06100 & -1.8858 \\
\hline $\mathrm{H}$ & 3.20140 & -1.40350 & -2.69272 \\
\hline
\end{tabular}




\begin{tabular}{|c|c|c|c|}
\hline $\mathrm{Cm}$ & 0.07279 & -0.01438 & -0.12981 \\
\hline 0 & -1.65810 & 0.63298 & -1.93046 \\
\hline $\mathrm{H}$ & -1.86573 & 1.52081 & -2.25790 \\
\hline $\mathrm{H}$ & -2.31690 & 0.04203 & -2.32526 \\
\hline O & -0.36320 & -1.44103 & 1.94487 \\
\hline $\mathrm{H}$ & 0.12378 & -2.22978 & 2.22585 \\
\hline $\mathrm{H}$ & -1.07939 & -1.33572 & 2.58805 \\
\hline O & 2.46866 & 0.82820 & -0.74179 \\
\hline $\mathrm{H}$ & 2.86585 & 0.84375 & -1.62614 \\
\hline $\mathrm{H}$ & 3.03928 & 1.38515 & -0.19048 \\
\hline O & -1.88887 & 0.90518 & 1.19368 \\
\hline $\mathrm{C}$ & -3.36007 & 0.93645 & 1.07026 \\
\hline $\mathrm{H}$ & -1.65638 & 1.27879 & 2.05635 \\
\hline O & 1.07057 & 1.21919 & 1.82799 \\
\hline $\mathrm{C}$ & 1.73389 & 0.90893 & 3.10896 \\
\hline $\mathrm{H}$ & 1.09644 & 2.17814 & 1.69817 \\
\hline O & -0.07776 & 2.54095 & -0.48085 \\
\hline $\mathrm{C}$ & 0.57964 & 3.61074 & -1.25569 \\
\hline $\mathrm{H}$ & -0.86823 & 2.91902 & -0.06942 \\
\hline O & -1.25564 & -2.01402 & -0.87821 \\
\hline $\mathrm{C}$ & -2.31770 & -2.87785 & -0.33501 \\
\hline $\mathrm{H}$ & -0.95550 & -2.39608 & -1.71547 \\
\hline O & 1.84403 & -1.78009 & 0.08209 \\
\hline C & 1.94277 & -3.22514 & 0.33736 \\
\hline $\mathrm{H}$ & 2.73981 & -1.41570 & 0.03307 \\
\hline O & 0.79108 & -0.71968 & -2.54095 \\
\hline $\mathrm{H}$ & 1.49332 & -1.32921 & -2.81610 \\
\hline $\mathrm{H}$ & 0.40588 & -0.37327 & -3.36087 \\
\hline $\mathrm{H}$ & 1.40208 & 3.14629 & -1.80287 \\
\hline $\mathrm{H}$ & 0.96094 & 4.37895 & -0.57470 \\
\hline $\mathrm{H}$ & -0.13478 & 4.05156 & -1.95887 \\
\hline $\mathrm{H}$ & -3.60278 & 0.51021 & 0.09517 \\
\hline $\mathrm{H}$ & -3.71063 & 1.97188 & 1.12781 \\
\hline $\mathrm{H}$ & -3.80980 & 0.33860 & 1.86964 \\
\hline $\mathrm{H}$ & 1.71524 & -0.17663 & 3.21753 \\
\hline $\mathrm{H}$ & 1.18516 & 1.38183 & 3.92981 \\
\hline $\mathrm{H}$ & 2.76721 & 1.26990 & 3.08425 \\
\hline $\mathrm{H}$ & -2.72237 & -2.36998 & 0.54272 \\
\hline $\mathrm{H}$ & -1.89962 & -3.84967 & -0.05320 \\
\hline $\mathrm{H}$ & -3.10300 & -3.00651 & -1.08680 \\
\hline $\mathrm{H}$ & 0.92739 & -3.62656 & 0.28487 \\
\hline $\mathrm{H}$ & 2.38171 & -3.39945 & 1.32633 \\
\hline $\mathrm{H}$ & 2.56306 & -3.69446 & -0.43296 \\
\hline
\end{tabular}




$\begin{array}{lrrr}\mathrm{Cm} & 0.04651 & -0.12284 & -0.04890 \\ \mathrm{O} & 1.14757 & 1.90699 & -1.08219 \\ \mathrm{C} & 2.24443 & 2.85070 & -0.80103 \\ \mathrm{H} & 0.64076 & 2.25443 & -1.82966 \\ \mathrm{O} & -2.48417 & -0.19842 & 0.02987 \\ \mathrm{C} & -3.63561 & -1.06280 & -0.28945 \\ \mathrm{H} & -2.82207 & 0.64086 & 0.37401 \\ \mathrm{O} & 1.32707 & -2.09536 & 0.94658 \\ \mathrm{C} & 2.57173 & -2.85327 & 0.70847 \\ \mathrm{H} & 0.87133 & -2.50334 & 1.69668 \\ \mathrm{O} & -0.99348 & 1.91109 & 1.11237 \\ \mathrm{C} & -1.21295 & 3.34234 & 0.82840 \\ \mathrm{H} & -1.17873 & 1.76587 & 2.05158 \\ \mathrm{O} & -0.80218 & -0.80308 & 2.33856 \\ \mathrm{H} & -1.69518 & -1.13105 & 2.52818 \\ \mathrm{H} & -0.35655 & -0.74898 & 3.19885 \\ \mathrm{O} & 1.78448 & 0.66236 & 1.62258 \\ \mathrm{H} & 2.48472 & 0.10194 & 1.99016 \\ \mathrm{H} & 1.95186 & 1.55377 & 1.96482 \\ \mathrm{O} & -0.92372 & 0.46578 & -2.37761 \\ \mathrm{H} & -1.84336 & 0.70072 & -2.57608 \\ \mathrm{H} & -0.49842 & 0.32571 & -3.23846 \\ \mathrm{O} & -0.65712 & -2.32318 & -1.13734 \\ \mathrm{H} & -1.23083 & -2.45525 & -1.90765 \\ \mathrm{H} & -0.42688 & -3.21151 & -0.82595 \\ \mathrm{O} & 1.79334 & -0.75897 & -1.77607 \\ \mathrm{H} & 1.95622 & -1.62179 & -2.18690 \\ \mathrm{H} & 2.46078 & -0.15688 & -2.13862 \\ \mathrm{H} & 2.99625 & 2.30785 & -0.22337 \\ \mathrm{H} & 1.86228 & 3.70913 & -0.23827 \\ \mathrm{H} & 2.68294 & 3.18628 & -1.74616 \\ \mathrm{H} & -3.25065 & -2.07675 & -0.41487 \\ \mathrm{H} & -4.11991 & -0.71458 & -1.20817 \\ \mathrm{H} & -4.34802 & -1.03938 & 0.54157 \\ \mathrm{H} & 3.19165 & -2.24792 & 0.04355 \\ \mathrm{H} & 2.33795 & -3.81880 & 0.24695 \\ \mathrm{H} & 3.08941 & -3.00939 & 1.66056 \\ \mathrm{H} & -1.14154 & 3.46693 & -0.25422 \\ \mathrm{H} & -0.45212 & 3.94348 & 1.33653 \\ \mathrm{H} & -2.21247 & 3.63099 & 1.16871 \\ & & & \end{array}$




$\begin{array}{lrrr}\mathrm{Cm} & 0.26608 & 0.02560 & 0.07585 \\ \mathrm{O} & -0.08671 & 2.37591 & -0.71634 \\ \mathrm{C} & -0.42419 & 3.71757 & -0.20750 \\ \mathrm{H} & -0.05293 & 2.42098 & -1.68292 \\ \mathrm{O} & -1.12819 & -1.52215 & -1.30980 \\ \mathrm{C} & -1.01966 & -2.65228 & -2.24500 \\ \mathrm{H} & -2.06266 & -1.39372 & -1.09319 \\ \mathrm{O} & 2.07030 & -0.42824 & 1.97170 \\ \mathrm{H} & 2.92093 & 0.02689 & 2.06938 \\ \mathrm{H} & 2.01694 & -1.04071 & 2.72137 \\ \mathrm{O} & -2.26887 & 0.43238 & 0.31616 \\ \mathrm{C} & -3.28217 & 1.30003 & -0.31672 \\ \mathrm{H} & -2.69803 & -0.03589 & 1.04666 \\ \mathrm{O} & -0.62706 & -1.58230 & 1.79258 \\ \mathrm{C} & -0.90460 & -3.03237 & 1.80382 \\ \mathrm{H} & -0.81857 & -1.23295 & 2.67509 \\ \mathrm{O} & -0.15387 & 1.34518 & 2.21524 \\ \mathrm{H} & 0.49841 & 1.49167 & 2.91775 \\ \mathrm{H} & -0.90646 & 1.91846 & 2.42712 \\ \mathrm{O} & 0.70008 & 0.32011 & -2.47989 \\ \mathrm{H} & 0.13757 & -0.00491 & -3.19969 \\ \mathrm{H} & 1.51795 & 0.62498 & -2.90285 \\ \mathrm{O} & 1.85516 & -1.85042 & -0.51348 \\ \mathrm{H} & 1.98902 & -2.34247 & -1.33778 \\ \mathrm{H} & 2.46230 & -2.24643 & 0.13020 \\ \mathrm{O} & 2.55693 & 1.18500 & -0.33336 \\ \mathrm{H} & 3.42841 & 0.80007 & -0.51533 \\ \mathrm{H} & 2.67873 & 2.14501 & -0.39586 \\ \mathrm{H} & -0.17565 & 3.72950 & 0.85576 \\ \mathrm{H} & -1.48966 & 3.91567 & -0.36175 \\ \mathrm{H} & 0.17955 & 4.46481 & -0.73211 \\ \mathrm{H} & -0.00920 & -2.63531 & -2.66198 \\ \mathrm{H} & -1.74946 & -2.52811 & -3.05165 \\ \mathrm{H} & -1.19569 & -3.59428 & -1.71552 \\ \mathrm{H} & -2.82020 & 1.72456 & -1.21046 \\ \mathrm{H} & -3.57772 & 2.09288 & 0.37797 \\ \mathrm{H} & -4.15385 & 0.69976 & -0.59630 \\ \mathrm{H} & -0.57814 & -3.42004 & 0.83692 \\ \mathrm{H} & -1.97735 & -3.20023 & 1.94323 \\ \mathrm{H} & -0.33705 & -3.50737 & 2.61034 \\ & & & \end{array}$




$\begin{array}{lrrr}\mathrm{Cm} & 0.33854 & -0.00306 & 0.05976 \\ \mathrm{O} & -0.46080 & -0.10929 & -2.33480 \\ \mathrm{C} & -0.27475 & 0.56648 & -3.63145 \\ \mathrm{H} & -1.21300 & -0.71199 & -2.41570 \\ \mathrm{O} & -1.21199 & 0.11768 & 2.05063 \\ \mathrm{C} & -1.47293 & -0.55896 & 3.33393 \\ \mathrm{H} & -1.94433 & 0.72558 & 1.87788 \\ \mathrm{O} & 2.92761 & -0.01775 & 0.50666 \\ \mathrm{H} & 3.60396 & -0.52467 & 0.03098 \\ \mathrm{H} & 3.40883 & 0.48598 & 1.18144 \\ \mathrm{O} & -1.33922 & 1.88374 & -0.15101 \\ \mathrm{C} & -2.51702 & 2.21420 & -0.97641 \\ \mathrm{H} & -1.10176 & 2.67384 & 0.35656 \\ \mathrm{O} & 1.01396 & 1.88010 & 1.69585 \\ \mathrm{H} & 0.71773 & 1.92944 & 2.61799 \\ \mathrm{H} & 1.59308 & 2.64643 & 1.56171 \\ \mathrm{O} & 1.57272 & 1.82392 & -1.22683 \\ \mathrm{H} & 2.53478 & 1.87994 & -1.33207 \\ \mathrm{H} & 1.20607 & 2.53029 & -1.78025 \\ \mathrm{O} & -1.33793 & -1.86745 & -0.30710 \\ \mathrm{C} & -2.73420 & -2.17084 & 0.06312 \\ \mathrm{H} & -0.95517 & -2.66571 & -0.70023 \\ \mathrm{O} & 1.05352 & -1.84388 & 1.67845 \\ \mathrm{H} & 0.51762 & -2.54624 & 2.07743 \\ \mathrm{H} & 1.92522 & -1.90768 & 2.09781 \\ \mathrm{O} & 1.50076 & -1.89526 & -1.25952 \\ \mathrm{H} & 1.99610 & -2.66562 & -0.94043 \\ \mathrm{H} & 1.52744 & -1.94463 & -2.22766 \\ \mathrm{H} & 0.76209 & 0.90735 & -3.67282 \\ \mathrm{H} & -0.96860 & 1.40979 & -3.71463 \\ \mathrm{H} & -0.45465 & -0.14932 & -4.43965 \\ \mathrm{H} & -0.51195 & -0.91107 & 3.71537 \\ \mathrm{H} & -2.16402 & -1.39463 & 3.18014 \\ \mathrm{H} & -1.90275 & 0.15954 & 4.03885 \\ \mathrm{H} & -2.81989 & 1.29365 & -1.47936 \\ \mathrm{H} & -2.25136 & 2.98291 & -1.70915 \\ \mathrm{H} & -3.32533 & 2.57022 & -0.32999 \\ \mathrm{H} & -3.17163 & -1.24281 & 0.43675 \\ \mathrm{H} & -2.75209 & -2.94328 & 0.83860 \\ \mathrm{H} & -3.27926 & -2.51100 & -0.82298\end{array}$




$\begin{array}{lrrr}\mathrm{Cm} & 0.16869 & -0.00332 & 0.10216 \\ \mathrm{O} & 0.47125 & 2.04918 & -1.34282 \\ \mathrm{C} & 0.82743 & 3.47379 & -1.21697 \\ \mathrm{H} & 0.16538 & 1.89750 & -2.24837 \\ \mathrm{O} & -1.95718 & -1.29163 & -0.28178 \\ \mathrm{C} & -2.47810 & -2.66615 & -0.40651 \\ \mathrm{H} & -2.70918 & -0.68204 & -0.28093 \\ \mathrm{O} & 2.05828 & -0.65164 & 1.90954 \\ \mathrm{H} & 3.01552 & -0.54555 & 1.79242 \\ \mathrm{H} & 1.94802 & -0.96881 & 2.81973 \\ \mathrm{O} & -1.96360 & 1.40491 & 0.41606 \\ \mathrm{C} & -2.70645 & 2.45879 & -0.30273 \\ \mathrm{H} & -2.35830 & 1.31599 & 1.29593 \\ \mathrm{O} & -0.87208 & -0.58388 & 2.38695 \\ \mathrm{H} & -1.46608 & -1.33099 & 2.55973 \\ \mathrm{H} & -0.74299 & -0.15048 & 3.24499 \\ \mathrm{O} & 0.74473 & 1.88224 & 1.70546 \\ \mathrm{H} & 1.51929 & 1.91535 & 2.28732 \\ \mathrm{H} & 0.30642 & 2.74195 & 1.79709 \\ \mathrm{O} & -0.12835 & -0.47818 & -2.40788 \\ \mathrm{H} & -0.93827 & -0.83383 & -2.80521 \\ \mathrm{H} & 0.55925 & -0.56996 & -3.08634 \\ \mathrm{O} & 0.96117 & -2.36341 & -0.05456 \\ \mathrm{C} & 0.98497 & -3.49472 & -0.99934 \\ \mathrm{H} & 1.49877 & -2.60580 & 0.71343 \\ \mathrm{O} & 2.51869 & 0.08714 & -0.90968 \\ \mathrm{H} & 3.16020 & -0.62384 & -1.06091 \\ \mathrm{H} & 2.90397 & 0.88043 & -1.31271 \\ \mathrm{H} & 1.45831 & 3.57060 & -0.33060 \\ \mathrm{H} & -0.08085 & 4.07733 & -1.11695 \\ \mathrm{H} & 1.39053 & 3.78486 & -2.10263 \\ \mathrm{H} & -1.65936 & -3.34132 & -0.15458 \\ \mathrm{H} & -2.82224 & -2.83946 & -1.43147 \\ \mathrm{H} & -3.30624 & -2.80436 & 0.29604 \\ \mathrm{H} & -2.34701 & 2.45327 & -1.33377 \\ \mathrm{H} & -2.51972 & 3.43051 & 0.16580 \\ \mathrm{H} & -3.77644 & 2.22903 & -0.28117 \\ \mathrm{H} & 0.34400 & -3.22236 & -1.83966 \\ \mathrm{H} & 0.60097 & -4.39206 & -0.50377 \\ \mathrm{H} & 2.01051 & -3.66346 & -1.34347 \\ & & & \end{array}$




$\begin{array}{lrrr}\mathrm{Cm} & 0.00139 & 0.00384 & 0.20415 \\ \mathrm{O} & -1.42738 & -1.69888 & -0.92751 \\ \mathrm{C} & -1.35423 & -2.97633 & -1.65656 \\ \mathrm{H} & -2.35898 & -1.44347 & -0.85019 \\ \mathrm{O} & -0.77856 & 1.91114 & -1.19354 \\ \mathrm{C} & -1.37227 & 3.25410 & -1.08076 \\ \mathrm{H} & -0.57789 & 1.74915 & -2.12681 \\ \mathrm{O} & 1.62544 & -0.09966 & 2.27001 \\ \mathrm{H} & 1.61447 & -0.69493 & 3.03494 \\ \mathrm{H} & 2.50520 & 0.30655 & 2.27776 \\ \mathrm{O} & 0.64054 & -0.20118 & -2.44009 \\ \mathrm{H} & 0.13989 & -0.70223 & -3.10321 \\ \mathrm{H} & 1.37145 & 0.20709 & -2.93088 \\ \mathrm{O} & 2.12774 & 1.27625 & -0.21144 \\ \mathrm{C} & 2.44314 & 2.71714 & -0.28883 \\ \mathrm{H} & 2.94170 & 0.78355 & -0.39034 \\ \mathrm{O} & 1.61489 & -1.81745 & -0.25884 \\ \mathrm{C} & 2.30160 & -2.88485 & 0.49348 \\ \mathrm{H} & 1.83715 & -1.91509 & -1.19586 \\ \mathrm{O} & -2.65258 & 0.39717 & 0.43978 \\ \mathrm{H} & -3.18894 & 1.01081 & -0.08553 \\ \mathrm{H} & -3.24213 & 0.08012 & 1.14194 \\ \mathrm{O} & -0.48044 & 1.71001 & 1.98561 \\ \mathrm{H} & -1.27684 & 2.23338 & 2.16129 \\ \mathrm{H} & 0.13705 & 1.90464 & 2.70755 \\ \mathrm{O} & -0.93085 & -1.50405 & 2.05900 \\ \mathrm{H} & -1.10788 & -1.30791 & 2.99239 \\ \mathrm{H} & -1.24462 & -2.41193 & 1.92440 \\ \mathrm{H} & -0.30030 & -3.25843 & -1.70305 \\ \mathrm{H} & -1.76487 & -2.84888 & -2.66404 \\ \mathrm{H} & -1.92009 & -3.74111 & -1.11469 \\ \mathrm{H} & -1.51409 & 3.45640 & -0.01700 \\ \mathrm{H} & -2.33205 & 3.27736 & -1.60775 \\ \mathrm{H} & -0.68869 & 3.99389 & -1.50938 \\ \mathrm{H} & 1.54547 & 3.25153 & 0.02849 \\ \mathrm{H} & 2.70494 & 2.98230 & -1.31816 \\ \mathrm{H} & 3.27389 & 2.94497 & 0.38647 \\ \mathrm{H} & 1.89527 & -2.87166 & 1.50666 \\ \mathrm{H} & 3.37818 & -2.68791 & 0.50892 \\ \mathrm{H} & 2.09500 & -3.85107 & 0.02262 \\ & & & \end{array}$




$\begin{array}{lrrr}\mathrm{Cm} & 0.18826 & -0.08150 & 0.13169 \\ \mathrm{O} & -0.75442 & -2.21436 & -0.80894 \\ \mathrm{C} & -0.38661 & -3.48900 & -1.45034 \\ \mathrm{H} & -1.71678 & -2.19424 & -0.70191 \\ \mathrm{O} & -0.91313 & 1.81354 & -1.03656 \\ \mathrm{C} & -1.59275 & 3.08834 & -0.75165 \\ \mathrm{H} & -0.87511 & 1.70282 & -1.99740 \\ \mathrm{O} & 2.02712 & -0.03076 & 2.02970 \\ \mathrm{H} & 2.12586 & -0.59035 & 2.81521 \\ \mathrm{H} & 2.78746 & 0.57047 & 2.04338 \\ \mathrm{O} & 0.47608 & -0.16147 & -2.47739 \\ \mathrm{H} & 0.03596 & -0.79295 & -3.06727 \\ \mathrm{H} & 1.09408 & 0.33106 & -3.04023 \\ \mathrm{O} & 2.06785 & 1.49219 & -0.41708 \\ \mathrm{C} & 2.22049 & 2.95679 & -0.51391 \\ \mathrm{H} & 2.90059 & 1.08335 & -0.69385 \\ \mathrm{O} & 2.25321 & -1.47399 & -0.44402 \\ \mathrm{H} & 2.89755 & -1.80346 & 0.20151 \\ \mathrm{H} & 2.49378 & -1.88269 & -1.28949 \\ \mathrm{O} & -2.35243 & -0.34521 & 0.51015 \\ \mathrm{C} & -3.61801 & 0.12553 & -0.08580 \\ \mathrm{H} & -2.56674 & -0.81036 & 1.33180 \\ \mathrm{O} & -0.44115 & 1.44159 & 2.03992 \\ \mathrm{H} & -1.30942 & 1.81018 & 2.26351 \\ \mathrm{H} & 0.16988 & 1.76781 & 2.71851 \\ \mathrm{O} & -0.33081 & -1.71567 & 2.10296 \\ \mathrm{H} & -0.43223 & -1.54462 & 3.05248 \\ \mathrm{H} & -0.39120 & -2.67932 & 2.00906 \\ \mathrm{H} & 0.70269 & -3.55918 & -1.42630 \\ \mathrm{H} & -0.75284 & -3.50455 & -2.48261 \\ \mathrm{H} & -0.82060 & -4.32043 & -0.88536 \\ \mathrm{H} & -1.43122 & 3.31109 & 0.30487 \\ \mathrm{H} & -2.66126 & 2.99307 & -0.96701 \\ \mathrm{H} & -1.15076 & 3.87922 & -1.36558 \\ \mathrm{H} & 1.32730 & 3.39644 & -0.06627 \\ \mathrm{H} & 2.30818 & 3.25056 & -1.56483 \\ \mathrm{H} & 3.10995 & 3.26719 & 0.04335 \\ \mathrm{H} & -3.37991 & 0.48736 & -1.08790 \\ \mathrm{H} & -4.04112 & 0.92944 & 0.52521 \\ \mathrm{H} & -4.32262 & -0.70976 & -0.14915\end{array}$




\begin{tabular}{|c|c|c|c|}
\hline $\mathrm{Cm}$ & -0.00464 & -0.18263 & 0.10353 \\
\hline 0 & -0.54507 & -2.28268 & -1.16934 \\
\hline C & -0.12244 & -3.68372 & -1.34107 \\
\hline $\mathrm{H}$ & -1.31125 & -2.12772 & -1.74146 \\
\hline 0 & 0.29482 & 1.37243 & -1.80622 \\
\hline C & -0.16094 & 2.67503 & -2.32336 \\
\hline $\mathrm{H}$ & 1.01183 & 1.05512 & -2.37504 \\
\hline O & 0.19787 & 0.04481 & 2.88004 \\
\hline $\mathrm{H}$ & -0.34155 & -0.38067 & 3.56504 \\
\hline $\mathrm{H}$ & 0.91538 & 0.48084 & 3.36566 \\
\hline O & 1.97868 & -0.84771 & -1.56444 \\
\hline $\mathrm{H}$ & 1.91943 & -1.53206 & -2.24907 \\
\hline $\mathrm{H}$ & 2.90607 & -0.56271 & -1.56865 \\
\hline O & 2.01986 & 1.10219 & 0.80199 \\
\hline C & 2.39953 & 2.52429 & 0.69530 \\
\hline $\mathrm{H}$ & 2.76399 & 0.61971 & 1.19005 \\
\hline O & 1.52311 & -1.88132 & 1.19046 \\
\hline $\mathrm{H}$ & 1.50002 & -2.12334 & 2.12910 \\
\hline $\mathrm{H}$ & 2.17235 & -2.47047 & 0.77699 \\
\hline O & -2.18573 & -0.02403 & -1.33146 \\
\hline $\mathrm{H}$ & -2.27325 & 0.44301 & -2.17661 \\
\hline $\mathrm{H}$ & -3.09047 & -0.24347 & -1.05903 \\
\hline 0 & -1.23189 & 1.69123 & 1.12494 \\
\hline $\mathrm{C}$ & -2.26379 & 2.70854 & 0.85254 \\
\hline $\mathrm{H}$ & -0.94774 & 1.78499 & 2.04701 \\
\hline O & -1.90645 & -1.37620 & 1.34785 \\
\hline $\mathrm{H}$ & -2.58962 & -1.00696 & 1.92860 \\
\hline $\mathrm{H}$ & -2.10535 & -2.32344 & 1.28465 \\
\hline $\mathrm{H}$ & 0.65720 & -3.87826 & -0.60147 \\
\hline $\mathrm{H}$ & 0.26121 & -3.83341 & -2.35623 \\
\hline $\mathrm{H}$ & -0.97425 & -4.34751 & -1.16055 \\
\hline $\mathrm{H}$ & -0.95181 & 3.02496 & -1.65762 \\
\hline $\mathrm{H}$ & -0.54419 & 2.54909 & -3.34140 \\
\hline $\mathrm{H}$ & 0.67449 & 3.38216 & -2.31874 \\
\hline $\mathrm{H}$ & 1.50944 & 3.06072 & 0.36033 \\
\hline $\mathrm{H}$ & 3.21126 & 2.63557 & -0.03063 \\
\hline $\mathrm{H}$ & 2.71263 & 2.89262 & 1.6772 \\
\hline $\mathrm{H}$ & -2.69504 & 2.47239 & -0.1220 \\
\hline $\mathrm{H}$ & -1.80336 & 3.70157 & 0.8431 \\
\hline $\mathrm{H}$ & -3.03777 & 2.65426 & 1.62422 \\
\hline
\end{tabular}




$\begin{array}{lrrr}\mathrm{Cm} & -0.01716 & -0.01477 & 0.10851 \\ \mathrm{O} & 0.93735 & -1.87426 & -1.31135 \\ \mathrm{C} & 2.03546 & -2.85760 & -1.33948 \\ \mathrm{H} & 0.45131 & -1.94206 & -2.14662 \\ \mathrm{O} & 0.16888 & 1.81048 & -1.58739 \\ \mathrm{C} & -0.62966 & 2.83397 & -2.28121 \\ \mathrm{H} & 1.09539 & 1.92725 & -1.84360 \\ \mathrm{O} & -0.92457 & -0.11843 & 2.58008 \\ \mathrm{H} & -1.48764 & -0.78675 & 2.99950 \\ \mathrm{H} & -0.75122 & 0.54046 & 3.26937 \\ \mathrm{O} & 2.48662 & 0.53443 & -0.65735 \\ \mathrm{H} & 3.00918 & 0.03444 & -1.30333 \\ \mathrm{H} & 3.09414 & 1.20064 & -0.29934 \\ \mathrm{O} & 0.81266 & 1.91461 & 1.48883 \\ \mathrm{C} & 0.54636 & 3.36674 & 1.46892 \\ \mathrm{H} & 1.48757 & 1.74401 & 2.16180 \\ \mathrm{O} & 1.66342 & -1.04777 & 1.69509 \\ \mathrm{H} & 1.44236 & -1.46564 & 2.54189 \\ \mathrm{H} & 2.61571 & -1.18010 & 1.57251 \\ \mathrm{O} & -1.44949 & -0.52944 & -2.04336 \\ \mathrm{H} & -1.45950 & -0.03087 & -2.87496 \\ \mathrm{H} & -2.16220 & -1.18266 & -2.12112 \\ \mathrm{O} & -2.25884 & 1.09720 & 0.32642 \\ \mathrm{H} & -2.93121 & 1.24030 & -0.35679 \\ \mathrm{H} & -2.65322 & 1.40601 & 1.15654 \\ \mathrm{O} & -1.34623 & -2.11652 & 0.47786 \\ \mathrm{C} & -2.61171 & -2.53774 & 1.10248 \\ \mathrm{H} & -0.88232 & -2.91131 & 0.17736 \\ \mathrm{H} & 2.50009 & -2.84708 & -0.35115 \\ \mathrm{H} & 2.76080 & -2.58342 & -2.11341 \\ \mathrm{H} & 1.62915 & -3.85342 & -1.54459 \\ \mathrm{H} & -1.65737 & 2.74016 & -1.92358 \\ \mathrm{H} & -0.58305 & 2.66688 & -3.36267 \\ \mathrm{H} & -0.24342 & 3.82869 & -2.03682 \\ \mathrm{H} & -0.28609 & 3.52273 & 0.77976 \\ \mathrm{H} & 1.43691 & 3.90024 & 1.12143 \\ \mathrm{H} & 0.27043 & 3.70052 & 2.47417 \\ \mathrm{H} & -3.18729 & -1.62956 & 1.29960 \\ \mathrm{H} & -2.40486 & -3.08292 & 2.03054 \\ \mathrm{H} & -3.16669 & -3.17961 & 0.41072 \\ & & & \end{array}$




\begin{tabular}{|c|c|c|c|}
\hline $\mathrm{Cm}$ & -0.22593 & 0.15762 & -0.12319 \\
\hline 0 & -0.38028 & -2.27444 & -0.74029 \\
\hline C & -0.16966 & -3.61062 & -0.15674 \\
\hline $\mathrm{H}$ & -0.56411 & -2.38456 & -1.68441 \\
\hline O & 0.98672 & 1.57203 & -1.89846 \\
\hline $\mathrm{H}$ & 0.71147 & 2.42395 & -2.27043 \\
\hline $\mathrm{H}$ & 1.89813 & 1.43758 & -2.19893 \\
\hline O & -1.47356 & 1.25271 & 1.86194 \\
\hline $\mathrm{H}$ & -2.34564 & 1.01310 & 2.21099 \\
\hline $\mathrm{H}$ & -1.09589 & 1.87052 & 2.50597 \\
\hline 0 & 2.17847 & -0.61654 & -0.44568 \\
\hline C & 2.91802 & -1.65123 & -1.19625 \\
\hline $\mathrm{H}$ & 2.82858 & -0.03607 & -0.02390 \\
\hline O & 1.31169 & 1.69279 & 1.18702 \\
\hline $\mathrm{C}$ & 1.84903 & 3.06364 & 1.06865 \\
\hline $\mathrm{H}$ & 1.58833 & 1.33743 & 2.04423 \\
\hline 0 & 0.50947 & -1.07245 & 1.93107 \\
\hline $\mathrm{C}$ & -0.01496 & -1.46250 & 3.25506 \\
\hline $\mathrm{H}$ & 1.33661 & -1.55482 & 1.78634 \\
\hline O & -1.10072 & -0.27265 & -2.56706 \\
\hline $\mathrm{H}$ & -0.63135 & -0.00538 & -3.37275 \\
\hline $\mathrm{H}$ & -1.97796 & -0.56420 & -2.86149 \\
\hline O & -1.66707 & 2.15935 & -0.83241 \\
\hline $\mathrm{H}$ & -2.05522 & 2.37484 & -1.69438 \\
\hline $\mathrm{H}$ & -2.01718 & 2.81965 & -0.21456 \\
\hline O & -2.65891 & -0.65490 & 0.05496 \\
\hline $\mathrm{H}$ & -3.49130 & -0.16103 & -0.00721 \\
\hline $\mathrm{H}$ & -2.91438 & -1.58765 & 0.12568 \\
\hline $\mathrm{H}$ & -0.18240 & -3.49025 & 0.92799 \\
\hline $\mathrm{H}$ & 0.79216 & -4.01475 & -0.48902 \\
\hline $\mathrm{H}$ & -0.98490 & -4.27304 & -0.46476 \\
\hline $\mathrm{H}$ & 2.17980 & -2.21215 & -1.77192 \\
\hline $\mathrm{H}$ & 3.44122 & -2.31012 & -0.49568 \\
\hline $\mathrm{H}$ & 3.63406 & -1.17265 & -1.87189 \\
\hline $\mathrm{H}$ & 1.45862 & 3.47636 & 0.1365 \\
\hline $\mathrm{H}$ & 2.94294 & 3.02831 & 1.04086 \\
\hline $\mathrm{H}$ & 1.50968 & 3.66588 & 1.91770 \\
\hline $\mathrm{H}$ & -0.97111 & -0.95195 & 3.3796 \\
\hline $\mathrm{H}$ & 0.68919 & -1.14838 & 4.0322 \\
\hline $\mathrm{H}$ & -0.16011 & -2.54714 & 3.28853 \\
\hline
\end{tabular}




$\begin{array}{lrrr}\text { Cm } & -0.07616 & 0.16483 & 0.16166 \\ \mathrm{O} & 0.31406 & -2.28070 & 0.65584 \\ \mathrm{C} & 1.33536 & -3.19234 & 1.20196 \\ \mathrm{H} & -0.37262 & -2.81632 & 0.23413 \\ \mathrm{O} & -0.93807 & 1.09667 & -2.04619 \\ \mathrm{H} & -1.66870 & 1.71746 & -2.18548 \\ \mathrm{H} & -0.57755 & 0.91197 & -2.92614 \\ \mathrm{O} & 0.28019 & 1.72882 & 2.21016 \\ \mathrm{H} & -0.00766 & 1.57751 & 3.12364 \\ \mathrm{H} & 0.73310 & 2.58606 & 2.21695 \\ \mathrm{O} & 1.37300 & -0.61084 & -1.72869 \\ \mathrm{C} & 1.63096 & -1.86020 & -2.46969 \\ \mathrm{H} & 1.95984 & 0.07224 & -2.08467 \\ \mathrm{O} & 1.41380 & 2.11185 & -0.46481 \\ \mathrm{C} & 1.32891 & 3.39465 & -1.19065 \\ \mathrm{H} & 2.30586 & 2.03365 & -0.09609 \\ \mathrm{O} & 2.29857 & -0.05742 & 1.16534 \\ \mathrm{H} & 2.55931 & 0.22670 & 2.05533 \\ \mathrm{H} & 3.01384 & -0.63481 & 0.85632 \\ \mathrm{O} & -2.03791 & -1.27916 & -0.54436 \\ \mathrm{C} & -2.67526 & -1.70065 & -1.80780 \\ \mathrm{H} & -2.62827 & -1.52232 & 0.18380 \\ \mathrm{O} & -2.11603 & 1.71318 & 0.50662 \\ \mathrm{H} & -3.03383 & 1.61126 & 0.21032 \\ \mathrm{H} & -2.10488 & 2.52986 & 1.02962 \\ \mathrm{O} & -1.39762 & -0.64297 & 2.26261 \\ \mathrm{H} & -2.11446 & -0.22015 & 2.76069 \\ \mathrm{H} & -1.19622 & -1.46436 & 2.73735 \\ \mathrm{H} & 1.86208 & -2.65278 & 1.99209 \\ \mathrm{H} & 2.02554 & -3.49681 & 0.40772 \\ \mathrm{H} & 0.84218 & -4.07112 & 1.62891 \\ \mathrm{H} & 0.91438 & -2.59864 & -2.10508 \\ \mathrm{H} & 2.65553 & -2.19554 & -2.27979 \\ \mathrm{H} & 1.47975 & -1.68754 & -3.53992 \\ \mathrm{H} & 0.29508 & 3.50243 & -1.52311 \\ \mathrm{H} & 2.00561 & 3.37539 & -2.05117 \\ \mathrm{H} & 1.59575 & 4.21460 & -0.51605 \\ \mathrm{H} & -1.93472 & -1.56199 & -2.59796 \\ \mathrm{H} & -3.56029 & -1.08599 & -2.00050 \\ \mathrm{H} & -2.95336 & -2.75700 & -1.73699 \\ & & & \end{array}$




$\begin{array}{lrrr}\mathrm{Cm} & -0.11079 & 0.01190 & 0.06198 \\ \mathrm{O} & -1.29889 & -2.22237 & -0.08154 \\ \mathrm{C} & -1.39087 & -3.50215 & 0.64378 \\ \mathrm{H} & -1.73785 & -2.33354 & -0.93685 \\ \mathrm{O} & 0.93181 & 0.98862 & -2.07834 \\ \mathrm{H} & 0.87379 & 1.88129 & -2.45139 \\ \mathrm{H} & 1.61846 & 0.53529 & -2.59002 \\ \mathrm{O} & -0.30541 & 1.17723 & 2.42345 \\ \mathrm{H} & -1.09721 & 1.18178 & 2.98380 \\ \mathrm{H} & 0.38803 & 1.60611 & 2.94864 \\ \mathrm{O} & 1.59445 & -1.55345 & -0.96745 \\ \mathrm{C} & 1.59637 & -2.78692 & -1.77810 \\ \mathrm{H} & 2.51588 & -1.32822 & -0.77330 \\ \mathrm{O} & 2.14953 & 0.84637 & 0.77420 \\ \mathrm{C} & 3.03087 & 1.98433 & 0.44779 \\ \mathrm{H} & 2.56754 & 0.33393 & 1.48160 \\ \mathrm{O} & 0.82606 & -1.43615 & 1.96787 \\ \mathrm{H} & 0.65299 & -1.31944 & 2.91492 \\ \mathrm{H} & 1.22027 & -2.31777 & 1.88001 \\ \mathrm{O} & -1.68901 & -0.15126 & -1.96804 \\ \mathrm{H} & -1.41345 & -0.04782 & -2.89220 \\ \mathrm{H} & -2.65662 & -0.08287 & -1.97253 \\ \mathrm{O} & -0.78465 & 2.41321 & -0.11659 \\ \mathrm{C} & -1.25898 & 3.36618 & -1.13445 \\ \mathrm{H} & -0.69047 & 2.89004 & 0.72081 \\ \mathrm{O} & -2.54286 & 0.15512 & 0.92017 \\ \mathrm{H} & -3.11875 & 0.92845 & 1.02307 \\ \mathrm{H} & -3.09515 & -0.61552 & 1.12315 \\ \mathrm{H} & -1.21234 & -3.28768 & 1.69977 \\ \mathrm{H} & -0.64492 & -4.20445 & 0.25625 \\ \mathrm{H} & -2.39676 & -3.91569 & 0.52053 \\ \mathrm{H} & 0.56132 & -2.97773 & -2.06821 \\ \mathrm{H} & 1.98737 & -3.61870 & -1.18319 \\ \mathrm{H} & 2.21210 & -2.63675 & -2.67062 \\ \mathrm{H} & 2.49359 & 2.60129 & -0.27496 \\ \mathrm{H} & 3.96472 & 1.61332 & 0.01334 \\ \mathrm{H} & 3.23563 & 2.56036 & 1.35594 \\ \mathrm{H} & -1.49248 & 2.78576 & -2.03063 \\ \mathrm{H} & -0.47849 & 4.10705 & -1.34003 \\ \mathrm{H} & -2.16507 & 3.86505 & -0.77610 \\ & & & \end{array}$




$\begin{array}{lrrr}\text { Cm } & 0.12945 & 0.01178 & -0.07687 \\ \mathrm{O} & -2.09158 & 1.27751 & -0.13488 \\ \mathrm{C} & -3.21957 & 1.58185 & 0.76577 \\ \mathrm{H} & -2.37497 & 1.46872 & -1.04052 \\ \mathrm{O} & 0.49991 & -1.61338 & -2.04731 \\ \mathrm{H} & 1.30015 & -1.73867 & -2.57995 \\ \mathrm{H} & -0.08571 & -2.35148 & -2.27380 \\ \mathrm{O} & 1.92945 & 0.52585 & 1.72883 \\ \mathrm{H} & 2.22133 & 1.39566 & 2.04186 \\ \mathrm{H} & 2.47219 & -0.11835 & 2.20821 \\ \mathrm{O} & -1.68057 & -1.75582 & -0.18747 \\ \mathrm{C} & -3.06933 & -1.91263 & -0.65890 \\ \mathrm{H} & -1.40092 & -2.60138 & 0.19186 \\ \mathrm{O} & 1.01849 & -2.12055 & 0.97999 \\ \mathrm{C} & 1.94318 & -3.21258 & 0.61433 \\ \mathrm{H} & 0.72172 & -2.27419 & 1.88899 \\ \mathrm{O} & -0.81208 & -0.20202 & 2.28292 \\ \mathrm{H} & -0.42871 & 0.19949 & 3.07836 \\ \mathrm{H} & -1.68653 & -0.52838 & 2.54592 \\ \mathrm{O} & -0.34317 & 1.08168 & -2.38380 \\ \mathrm{H} & -0.44489 & 0.58486 & -3.21077 \\ \mathrm{H} & -0.30004 & 2.01648 & -2.63931 \\ \mathrm{O} & 2.40638 & 0.38403 & -1.17772 \\ \mathrm{H} & 2.58800 & 0.76057 & -2.05273 \\ \mathrm{H} & 3.26847 & 0.32454 & -0.73788 \\ \mathrm{O} & 0.39958 & 2.48797 & 0.21082 \\ \mathrm{C} & 1.44810 & 3.51620 & 0.30815 \\ \mathrm{H} & -0.46068 & 2.91655 & 0.32707 \\ \mathrm{H} & -2.80824 & 1.67485 & 1.77351 \\ \mathrm{H} & -3.96188 & 0.77830 & 0.71940 \\ \mathrm{H} & -3.67295 & 2.53237 & 0.46721 \\ \mathrm{H} & -3.32397 & -1.00420 & -1.20821 \\ \mathrm{H} & -3.73763 & -2.04495 & 0.19820 \\ \mathrm{H} & -3.13356 & -2.77858 & -1.32540 \\ \mathrm{H} & 2.34822 & -2.96768 & -0.36948 \\ \mathrm{H} & 1.39689 & -4.16065 & 0.57614 \\ \mathrm{H} & 2.75186 & -3.26976 & 1.35013 \\ \mathrm{H} & 2.39539 & 3.03066 & 0.06085 \\ \mathrm{H} & 1.47192 & 3.92603 & 1.32426 \\ \mathrm{H} & 1.24704 & 4.31531 & -0.41255 \\ & & & \end{array}$




$\begin{array}{lrrr}\mathrm{Cm} & 0.31575 & -0.05555 & 0.02256 \\ \mathrm{O} & -1.73648 & 0.46077 & -1.33831 \\ \mathrm{C} & -2.63862 & 1.57244 & -1.68445 \\ \mathrm{H} & -2.11617 & -0.35839 & -1.68697 \\ \mathrm{O} & 0.55758 & -2.00465 & 1.69425 \\ \mathrm{H} & 1.26138 & -2.67007 & 1.65763 \\ \mathrm{H} & -0.07112 & -2.32353 & 2.35945 \\ \mathrm{O} & 2.61443 & 1.11844 & -0.17852 \\ \mathrm{H} & 3.03748 & 1.50372 & -0.96129 \\ \mathrm{H} & 3.13022 & 1.43846 & 0.57736 \\ \mathrm{O} & -1.53648 & 0.07681 & 1.70544 \\ \mathrm{C} & -2.99554 & -0.13894 & 1.76272 \\ \mathrm{H} & -1.23121 & 0.29480 & 2.59844 \\ \mathrm{O} & 1.25703 & 0.67141 & 2.35412 \\ \mathrm{H} & 1.62840 & 0.06909 & 3.01770 \\ \mathrm{H} & 1.36633 & 1.56432 & 2.71652 \\ \mathrm{O} & -0.13733 & 2.38575 & 0.33028 \\ \mathrm{C} & 0.40191 & 3.67589 & -0.13954 \\ \mathrm{H} & -0.93689 & 2.57084 & 0.84373 \\ \mathrm{O} & -0.67097 & -2.10188 & -1.16153 \\ \mathrm{C} & -1.40896 & -3.30458 & -0.72698 \\ \mathrm{H} & -0.39538 & -2.24064 & -2.07949 \\ \mathrm{O} & 2.29890 & -1.65705 & -0.54155 \\ \mathrm{H} & 2.31787 & -2.56483 & -0.88150 \\ \mathrm{H} & 3.22675 & -1.38202 & -0.48003 \\ \mathrm{O} & 0.90835 & 0.23479 & -2.44552 \\ \mathrm{H} & 1.68617 & -0.05815 & -2.94535 \\ \mathrm{H} & 0.33611 & 0.68422 & -3.08672 \\ \mathrm{H} & -2.09213 & 2.49767 & -1.49458 \\ \mathrm{H} & -3.54226 & 1.52421 & -1.06783 \\ \mathrm{H} & -2.90045 & 1.50889 & -2.74548 \\ \mathrm{H} & -3.30797 & -0.44823 & 0.76386 \\ \mathrm{H} & -3.49265 & 0.79411 & 2.04646 \\ \mathrm{H} & -3.21983 & -0.92588 & 2.48963 \\ \mathrm{H} & 1.21899 & 3.44841 & -0.82647 \\ \mathrm{H} & 0.76987 & 4.25309 & 0.71489 \\ \mathrm{H} & -0.38156 & 4.23212 & -0.66435 \\ \mathrm{H} & -1.87150 & -3.06239 & 0.23261 \\ \mathrm{H} & -0.71847 & -4.14711 & -0.61837 \\ \mathrm{H} & -2.18394 & -3.54050 & -1.46322 \\ & & & \end{array}$




$\begin{array}{lrrr}\mathrm{Cm} & 0.03620 & -0.16225 & -0.00543 \\ \mathrm{O} & -0.63729 & 1.56929 & -1.69853 \\ \mathrm{C} & -0.33433 & 2.95893 & -2.08237 \\ \mathrm{H} & -1.37598 & 1.26045 & -2.24287 \\ \mathrm{O} & -1.90201 & -1.40443 & 1.17225 \\ \mathrm{H} & -2.03239 & -2.36112 & 1.25905 \\ \mathrm{H} & -2.64938 & -0.99666 & 1.63529 \\ \mathrm{O} & 2.52529 & -0.91853 & 0.42807 \\ \mathrm{H} & 3.24384 & -0.91419 & -0.22331 \\ \mathrm{H} & 2.96434 & -0.98619 & 1.29004 \\ \mathrm{O} & -1.46912 & 1.44729 & 1.26931 \\ \mathrm{C} & -2.56159 & 2.39730 & 0.97620 \\ \mathrm{H} & -1.37022 & 1.39851 & 2.23144 \\ \mathrm{O} & 0.51176 & -0.34267 & 2.53458 \\ \mathrm{H} & 0.15656 & -1.02284 & 3.12835 \\ \mathrm{H} & 1.11896 & 0.18386 & 3.07754 \\ \mathrm{O} & 1.35121 & 1.87142 & 0.59327 \\ \mathrm{C} & 2.71678 & 2.41392 & 0.46595 \\ \mathrm{H} & 0.79111 & 2.56043 & 0.97877 \\ \mathrm{O} & -1.74400 & -0.97710 & -1.73178 \\ \mathrm{H} & -2.65917 & -1.22745 & -1.53039 \\ \mathrm{H} & -1.57377 & -1.31850 & -2.62385 \\ \mathrm{O} & 0.43583 & -2.63965 & -0.11340 \\ \mathrm{C} & -0.25832 & -3.89958 & -0.42289 \\ \mathrm{H} & 1.33477 & -2.85163 & 0.17822 \\ \mathrm{O} & 1.28773 & -0.54928 & -2.19925 \\ \mathrm{H} & 1.67165 & -1.36735 & -2.55148 \\ \mathrm{H} & 1.38610 & 0.11176 & -2.90202 \\ \mathrm{H} & 0.60510 & 3.22539 & -1.59544 \\ \mathrm{H} & -1.14121 & 3.62026 & -1.75002 \\ \mathrm{H} & -0.22037 & 3.01829 & -3.16951 \\ \mathrm{H} & -2.73580 & 2.35447 & -0.10023 \\ \mathrm{H} & -2.26129 & 3.40630 & 1.27675 \\ \mathrm{H} & -3.46489 & 2.09437 & 1.51518 \\ \mathrm{H} & 3.27459 & 1.73177 & -0.17841 \\ \mathrm{H} & 3.18149 & 2.47650 & 1.45520 \\ \mathrm{H} & 2.67371 & 3.40559 & 0.00437 \\ \mathrm{H} & -1.23703 & -3.63449 & -0.83038 \\ \mathrm{H} & -0.36552 & -4.49670 & 0.48961 \\ \mathrm{H} & 0.31103 & -4.45897 & -1.17217 \\ & & & \end{array}$




\begin{tabular}{|c|c|c|c|}
\hline $\mathrm{Cm}$ & 0.18888 & -0.03281 & -0.10481 \\
\hline 0 & -1.69014 & -0.42215 & -1.82552 \\
\hline $\mathrm{H}$ & -2.34411 & 0.21843 & -2.14302 \\
\hline $\mathrm{H}$ & -1.96826 & -1.28107 & -2.17801 \\
\hline O & 0.68931 & -1.42164 & 1.96748 \\
\hline $\mathrm{H}$ & 1.55248 & -1.79481 & 2.20101 \\
\hline $\mathrm{H}$ & 0.08381 & -1.69526 & 2.67245 \\
\hline O & 1.88502 & 1.83515 & -0.62078 \\
\hline $\mathrm{H}$ & 2.28506 & 2.06447 & -1.47353 \\
\hline $\mathrm{H}$ & 2.14466 & 2.54247 & -0.01119 \\
\hline O & -1.90270 & -0.25408 & 1.27025 \\
\hline C & -3.16979 & -1.01170 & 1.21947 \\
\hline $\mathrm{H}$ & -1.88025 & 0.23284 & 2.10710 \\
\hline O & 0.39682 & 1.56774 & 1.85501 \\
\hline $\mathrm{C}$ & 1.08032 & 1.67039 & 3.16046 \\
\hline $\mathrm{H}$ & -0.05250 & 2.40864 & 1.68629 \\
\hline O & -1.20420 & 2.08368 & -0.50815 \\
\hline $\mathrm{C}$ & -1.12126 & 3.31094 & -1.32650 \\
\hline $\mathrm{H}$ & -2.08817 & 2.05411 & -0.11408 \\
\hline O & 0.09630 & -2.44314 & -0.82771 \\
\hline $\mathrm{C}$ & -0.28775 & -3.73764 & -0.23316 \\
\hline $\mathrm{H}$ & 0.45591 & -2.61522 & -1.70999 \\
\hline O & 2.64103 & -0.86871 & -0.03974 \\
\hline $\mathrm{H}$ & 2.99489 & -1.76768 & -0.12337 \\
\hline $\mathrm{H}$ & 3.41537 & -0.28914 & 0.03125 \\
\hline O & 1.06711 & -0.21364 & -2.53939 \\
\hline $\mathrm{H}$ & 1.96870 & -0.35705 & -2.86693 \\
\hline $\mathrm{H}$ & 0.51518 & -0.10121 & -3.32907 \\
\hline $\mathrm{H}$ & -0.13270 & 3.31487 & -1.78909 \\
\hline $\mathrm{H}$ & -1.24303 & 4.18891 & -0.68375 \\
\hline $\mathrm{H}$ & -1.89863 & 3.29473 & -2.09749 \\
\hline $\mathrm{H}$ & -3.15790 & -1.58375 & 0.28998 \\
\hline $\mathrm{H}$ & -4.01342 & -0.31427 & 1.22489 \\
\hline $\mathrm{H}$ & -3.22759 & -1.68655 & 2.07950 \\
\hline $\mathrm{H}$ & 1.63074 & 0.73906 & 3.30250 \\
\hline $\mathrm{H}$ & 0.33705 & 1.79897 & 3.95405 \\
\hline $\mathrm{H}$ & 1.77144 & 2.51947 & 3.14678 \\
\hline $\mathrm{H}$ & -0.79905 & -3.51564 & 0.70548 \\
\hline $\mathrm{H}$ & 0.60752 & -4.33937 & -0.04637 \\
\hline $\mathrm{H}$ & -0.96476 & -4.26374 & -0.91374 \\
\hline
\end{tabular}




\begin{tabular}{|c|c|c|c|}
\hline $\mathrm{Cm}$ & 0.05244 & -0.04016 & 0.20290 \\
\hline 0 & -1.74950 & -0.90603 & 1.83091 \\
\hline $\mathrm{H}$ & -1.67924 & -1.67690 & 2.41400 \\
\hline $\mathrm{H}$ & -2.67621 & -0.62546 & 1.87611 \\
\hline O & -0.11829 & 0.51392 & -2.27524 \\
\hline $\mathrm{H}$ & 0.15858 & 1.31782 & -2.73952 \\
\hline $\mathrm{H}$ & -0.51453 & -0.05519 & -2.95141 \\
\hline O & 2.20996 & 0.24360 & 1.66219 \\
\hline $\mathrm{H}$ & 2.25544 & 0.64848 & 2.54233 \\
\hline $\mathrm{H}$ & 3.10499 & -0.08123 & 1.47787 \\
\hline O & -1.01834 & -1.93606 & -1.05137 \\
\hline C & -2.37001 & -2.52116 & -1.15468 \\
\hline $\mathrm{H}$ & -0.42242 & -2.46988 & -1.59689 \\
\hline O & 1.95903 & -1.21757 & -0.92331 \\
\hline $\mathrm{C}$ & 2.86087 & -1.00179 & -2.07212 \\
\hline $\mathrm{H}$ & 2.23271 & -2.02990 & -0.47300 \\
\hline O & 0.74496 & -2.28387 & 1.45391 \\
\hline $\mathrm{H}$ & 1.34366 & -2.35269 & 2.21410 \\
\hline $\mathrm{H}$ & 0.37952 & -3.17452 & 1.33488 \\
\hline O & -2.02591 & 1.30731 & -0.07612 \\
\hline $\mathrm{C}$ & -3.07706 & 1.50750 & -1.09050 \\
\hline $\mathrm{H}$ & -2.16996 & 1.94644 & 0.63690 \\
\hline O & 1.17923 & 2.14967 & -0.24036 \\
\hline $\mathrm{C}$ & 0.89185 & 3.39366 & -0.97446 \\
\hline $\mathrm{H}$ & 2.07473 & 2.21292 & 0.12240 \\
\hline O & -0.33011 & 1.52119 & 2.27941 \\
\hline $\mathrm{H}$ & 0.00287 & 2.41825 & 2.43716 \\
\hline $\mathrm{H}$ & -0.80637 & 1.27066 & 3.08641 \\
\hline $\mathrm{H}$ & -3.04211 & -1.86282 & -0.60072 \\
\hline $\mathrm{H}$ & -2.37221 & -3.52384 & -0.71559 \\
\hline $\mathrm{H}$ & -2.66867 & -2.56514 & -2.20697 \\
\hline $\mathrm{H}$ & 2.57574 & -0.04920 & -2.52148 \\
\hline $\mathrm{H}$ & 2.73618 & -1.81579 & -2.79345 \\
\hline $\mathrm{H}$ & 3.89644 & -0.95961 & -1.71965 \\
\hline $\mathrm{H}$ & -2.94416 & 0.72841 & -1.84339 \\
\hline $\mathrm{H}$ & -2.96860 & 2.49761 & -1.54445 \\
\hline $\mathrm{H}$ & -4.05979 & 1.41037 & -0.61822 \\
\hline $\mathrm{H}$ & -0.15736 & 3.34696 & -1.27764 \\
\hline $\mathrm{H}$ & 1.54991 & 3.46996 & -1.84735 \\
\hline $\mathrm{H}$ & 1.04561 & 4.25364 & -0.31485 \\
\hline
\end{tabular}




\begin{tabular}{|c|c|c|c|}
\hline $\mathrm{Cm}$ & 0.08900 & 0.02853 & 0.00071 \\
\hline 0 & -1.54943 & 0.61321 & 1.97543 \\
\hline $\mathrm{H}$ & -1.43958 & 0.40254 & 2.91541 \\
\hline $\mathrm{H}$ & -2.48350 & 0.85254 & 1.87383 \\
\hline O & -0.52015 & -1.23865 & -2.11588 \\
\hline $\mathrm{H}$ & -0.32576 & -0.97136 & -3.02676 \\
\hline $\mathrm{H}$ & -0.98611 & -2.08565 & -2.17897 \\
\hline 0 & 2.53146 & 0.85948 & 0.23039 \\
\hline $\mathrm{H}$ & 2.84294 & 1.74761 & 0.46271 \\
\hline $\mathrm{H}$ & 3.33177 & 0.33479 & 0.07649 \\
\hline O & -1.10010 & -2.09687 & 0.59341 \\
\hline $\mathrm{C}$ & -2.43794 & -2.51786 & 1.05112 \\
\hline $\mathrm{H}$ & -0.54586 & -2.88738 & 0.52153 \\
\hline O & 1.74791 & -1.89127 & -0.22187 \\
\hline $\mathrm{C}$ & 2.35577 & -2.72265 & -1.28121 \\
\hline $\mathrm{H}$ & 2.16459 & -2.13150 & 0.61848 \\
\hline O & 1.08075 & -0.63917 & 2.32386 \\
\hline $\mathrm{H}$ & 1.82190 & -0.18688 & 2.75694 \\
\hline $\mathrm{H}$ & 0.76211 & -1.28737 & 2.97141 \\
\hline 0 & -1.98570 & 1.11754 & -0.87963 \\
\hline $\mathrm{C}$ & -3.17891 & 0.74624 & -1.66554 \\
\hline $\mathrm{H}$ & -2.02249 & 2.07048 & -0.71117 \\
\hline O & 0.83920 & 1.25201 & -2.12884 \\
\hline $\mathrm{H}$ & 0.28742 & 1.73200 & -2.76563 \\
\hline $\mathrm{H}$ & 1.75493 & 1.41848 & -2.40081 \\
\hline O & 0.10650 & 2.45187 & 0.72624 \\
\hline $\mathrm{C}$ & 0.75873 & 3.73034 & 0.39694 \\
\hline $\mathrm{H}$ & -0.38912 & 2.56663 & 1.55066 \\
\hline $\mathrm{H}$ & -3.08896 & -1.64440 & 0.97618 \\
\hline $\mathrm{H}$ & -2.38121 & -2.86570 & 2.08754 \\
\hline $\mathrm{H}$ & -2.81058 & -3.31612 & 0.40130 \\
\hline $\mathrm{H}$ & 1.93345 & -2.38440 & -2.22879 \\
\hline $\mathrm{H}$ & 2.10993 & -3.77542 & -1.10766 \\
\hline $\mathrm{H}$ & 3.44152 & -2.58160 & -1.27960 \\
\hline $\mathrm{H}$ & -3.15729 & -0.33905 & -1.77868 \\
\hline $\mathrm{H}$ & -3.14009 & 1.23177 & -2.64600 \\
\hline $\mathrm{H}$ & -4.07989 & 1.05250 & -1.12445 \\
\hline $\mathrm{H}$ & 1.24798 & 3.59847 & -0.57107 \\
\hline $\mathrm{H}$ & 1.48903 & 3.98564 & 1.17320 \\
\hline $\mathrm{H}$ & 0.00337 & 4.51930 & 0.32282 \\
\hline
\end{tabular}




$\begin{array}{lrrr}\mathrm{Cm} & 0.02530 & -0.01039 & 0.00904 \\ \mathrm{O} & -1.76774 & 1.63913 & -0.65532 \\ \mathrm{C} & -2.25130 & 2.99562 & -0.32951 \\ \mathrm{H} & -2.32254 & 1.28713 & -1.36670 \\ \mathrm{O} & -0.99213 & -2.30875 & 0.22272 \\ \mathrm{C} & -0.74853 & -3.71074 & -0.17059 \\ \mathrm{H} & -1.81730 & -2.28039 & 0.72864 \\ \mathrm{O} & 2.48544 & 0.44182 & 0.50516 \\ \mathrm{C} & 3.58026 & 1.33176 & 0.06858 \\ \mathrm{H} & 2.83832 & -0.14632 & 1.18824 \\ \mathrm{O} & -1.99939 & -0.10400 & 1.66618 \\ \mathrm{H} & -2.86065 & 0.31689 & 1.51945 \\ \mathrm{H} & -2.01715 & -0.41549 & 2.58495 \\ \mathrm{O} & 0.84154 & -1.12183 & 2.20551 \\ \mathrm{H} & 0.81195 & -2.07366 & 2.39031 \\ \mathrm{H} & 1.09468 & -0.70348 & 3.04370 \\ \mathrm{O} & 0.27444 & 1.88894 & 1.65748 \\ \mathrm{H} & 1.09746 & 2.37022 & 1.83334 \\ \mathrm{H} & -0.39775 & 2.29740 & 2.22441 \\ \mathrm{O} & -1.24518 & -0.67356 & -2.18108 \\ \mathrm{H} & -1.85035 & -1.42353 & -2.28958 \\ \mathrm{H} & -1.10325 & -0.32162 & -3.07420 \\ \mathrm{O} & 1.52355 & -1.50158 & -1.38383 \\ \mathrm{H} & 1.27539 & -2.05330 & -2.14160 \\ \mathrm{H} & 2.47629 & -1.63230 & -1.26160 \\ \mathrm{O} & 0.87265 & 1.45885 & -1.87441 \\ \mathrm{H} & 1.65197 & 1.32833 & -2.43666 \\ \mathrm{H} & 0.49231 & 2.30931 & -2.14309 \\ \mathrm{H} & -1.47993 & 3.47377 & 0.27811 \\ \mathrm{H} & -3.19620 & 2.93136 & 0.22063 \\ \mathrm{H} & -2.39493 & 3.56127 & -1.25588 \\ \mathrm{H} & 0.27628 & -3.76516 & -0.54350 \\ \mathrm{H} & -1.46130 & -4.00761 & -0.94744 \\ \mathrm{H} & -0.85865 & -4.35737 & 0.70606 \\ \mathrm{H} & 3.12261 & 2.15751 & -0.48089 \\ \mathrm{H} & 4.27615 & 0.77918 & -0.57187 \\ \mathrm{H} & 4.10615 & 1.71759 & 0.94798 \\ & & & \end{array}$




$\begin{array}{lrrr}\mathrm{Cm} & 0.26113 & -0.09740 & 0.05701 \\ \mathrm{O} & 0.00084 & 2.11179 & -1.10061 \\ \mathrm{C} & 0.11942 & 3.55485 & -0.81575 \\ \mathrm{H} & -0.36294 & 2.01453 & -1.99231 \\ \mathrm{O} & -1.64379 & -1.70682 & -0.30032 \\ \mathrm{C} & -1.94187 & -3.09466 & -0.70417 \\ \mathrm{H} & -2.48122 & -1.26758 & -0.09267 \\ \mathrm{O} & 2.40259 & -0.68091 & 1.45872 \\ \mathrm{H} & 3.31263 & -0.42685 & 1.23949 \\ \mathrm{H} & 2.46906 & -1.19781 & 2.27665 \\ \mathrm{O} & -1.99049 & 0.82379 & 0.84284 \\ \mathrm{C} & -3.00309 & 1.79772 & 0.38648 \\ \mathrm{H} & -2.22546 & 0.55500 & 1.74317 \\ \mathrm{O} & -0.36035 & -1.18741 & 2.31153 \\ \mathrm{H} & -0.82253 & -2.03496 & 2.40781 \\ \mathrm{H} & -0.16820 & -0.89252 & 3.21566 \\ \mathrm{O} & 0.76317 & 1.62442 & 1.84438 \\ \mathrm{H} & 1.59508 & 1.69404 & 2.33777 \\ \mathrm{H} & 0.21713 & 2.36983 & 2.13799 \\ \mathrm{O} & -0.23186 & -0.35504 & -2.47232 \\ \mathrm{H} & -1.02452 & -0.76332 & -2.85384 \\ \mathrm{H} & 0.37043 & -0.20731 & -3.21877 \\ \mathrm{O} & 1.34782 & -2.26970 & -0.69622 \\ \mathrm{H} & 1.18527 & -2.77843 & -1.50542 \\ \mathrm{H} & 2.00263 & -2.77764 & -0.19278 \\ \mathrm{O} & 2.41835 & 0.51464 & -1.19005 \\ \mathrm{H} & 3.11484 & -0.06828 & -1.53044 \\ \mathrm{H} & 2.65159 & 1.40624 & -1.49199 \\ \mathrm{H} & 0.79928 & 3.65973 & 0.03246 \\ \mathrm{H} & -0.86667 & 3.96804 & -0.58019 \\ \mathrm{H} & 0.54250 & 4.06025 & -1.68966 \\ \mathrm{H} & -0.99712 & -3.64189 & -0.69562 \\ \mathrm{H} & -2.38773 & -3.10286 & -1.70454 \\ \mathrm{H} & -2.63244 & -3.54139 & 0.01851 \\ \mathrm{H} & -2.80642 & 1.98767 & -0.67058 \\ \mathrm{H} & -2.91707 & 2.72143 & 0.96776 \\ \mathrm{H} & -4.00172 & 1.36644 & 0.50794 \\ & & & \end{array}$




$\begin{array}{lrrr}\mathrm{Cm} & 0.03321 & -0.09350 & 0.18628 \\ \mathrm{O} & -0.52618 & -2.21632 & -1.02445 \\ \mathrm{C} & 0.07539 & -3.48413 & -1.48052 \\ \mathrm{H} & -1.46421 & -2.22696 & -1.26599 \\ \mathrm{O} & -0.81385 & 1.60390 & -1.42899 \\ \mathrm{C} & -1.69151 & 2.78507 & -1.51014 \\ \mathrm{H} & -0.37646 & 1.49649 & -2.28640 \\ \mathrm{O} & 1.15755 & 0.07279 & 2.56993 \\ \mathrm{H} & 1.00129 & -0.48183 & 3.34982 \\ \mathrm{H} & 1.83643 & 0.71035 & 2.83937 \\ \mathrm{O} & 1.22122 & -0.17557 & -2.17793 \\ \mathrm{H} & 1.03602 & -0.82676 & -2.87247 \\ \mathrm{H} & 1.97581 & 0.34004 & -2.50384 \\ \mathrm{O} & 1.83563 & 1.63860 & 0.22066 \\ \mathrm{C} & 1.84328 & 3.11367 & 0.13973 \\ \mathrm{H} & 2.75510 & 1.33616 & 0.24901 \\ \mathrm{O} & 2.19950 & -1.39383 & 0.34504 \\ \mathrm{H} & 2.62197 & -1.66954 & 1.17349 \\ \mathrm{H} & 2.73369 & -1.77734 & -0.36736 \\ \mathrm{O} & -2.54276 & -0.38273 & -0.27814 \\ \mathrm{H} & -3.05141 & 0.07745 & -0.96365 \\ \mathrm{H} & -3.19645 & -0.86804 & 0.24955 \\ \mathrm{O} & -1.24075 & 1.44044 & 1.71937 \\ \mathrm{H} & -2.16624 & 1.72423 & 1.66602 \\ \mathrm{H} & -0.88811 & 1.83719 & 2.53106 \\ \mathrm{O} & -0.98848 & -1.73490 & 1.89527 \\ \mathrm{H} & -1.45682 & -1.55664 & 2.72591 \\ \mathrm{H} & -1.00072 & -2.69979 & 1.79643 \\ \mathrm{H} & 1.09236 & -3.51781 & -1.08429 \\ \mathrm{H} & 0.08366 & -3.51648 & -2.57533 \\ \mathrm{H} & -0.50306 & -4.32493 & -1.08404 \\ \mathrm{H} & -2.09961 & 2.95386 & -0.51139 \\ \mathrm{H} & -2.49681 & 2.59587 & -2.22781 \\ \mathrm{H} & -1.10484 & 3.65498 & -1.82211 \\ \mathrm{H} & 0.80422 & 3.43766 & 0.22612 \\ \mathrm{H} & 2.26538 & 3.42706 & -0.82044 \\ \mathrm{H} & 2.43378 & 3.51968 & 0.96702 \\ & & & \end{array}$




\begin{tabular}{|c|c|c|c|}
\hline $\mathrm{Cm}$ & -0.19427 & 0.08907 & -0.03770 \\
\hline 0 & -0.39028 & -2.42243 & -0.01735 \\
\hline C & -0.22741 & -3.55251 & 0.91782 \\
\hline $\mathrm{H}$ & -0.48054 & -2.78794 & -0.90898 \\
\hline 0 & 0.99432 & 1.19799 & -2.03431 \\
\hline $\mathrm{H}$ & 0.73175 & 1.98009 & -2.54363 \\
\hline $\mathrm{H}$ & 1.90862 & 1.01056 & -2.29575 \\
\hline 0 & -1.45411 & 1.35823 & 1.84781 \\
\hline $\mathrm{H}$ & -2.34140 & 1.15273 & 2.18103 \\
\hline $\mathrm{H}$ & -1.15650 & 2.13034 & 2.35317 \\
\hline O & 2.17341 & -0.73934 & -0.28009 \\
\hline $\mathrm{C}$ & 2.87566 & -1.93835 & -0.78054 \\
\hline $\mathrm{H}$ & 2.84281 & -0.12931 & 0.06247 \\
\hline 0 & 1.29932 & 1.84929 & 0.96988 \\
\hline $\mathrm{C}$ & 1.80115 & 3.19170 & 0.61024 \\
\hline $\mathrm{H}$ & 1.62855 & 1.63521 & 1.85523 \\
\hline 0 & 0.55160 & -0.64387 & 2.29454 \\
\hline $\mathrm{H}$ & 0.06645 & -0.47905 & 3.11827 \\
\hline $\mathrm{H}$ & 1.23949 & -1.29005 & 2.51813 \\
\hline 0 & -0.97491 & -0.83378 & -2.31506 \\
\hline $\mathrm{H}$ & -0.50360 & -0.69500 & -3.15161 \\
\hline $\mathrm{H}$ & -1.83004 & -1.22394 & -2.55558 \\
\hline 0 & -1.64877 & 1.94822 & -1.04008 \\
\hline $\mathrm{H}$ & -2.03695 & 2.01109 & -1.92685 \\
\hline $\mathrm{H}$ & -1.99750 & 2.71079 & -0.55250 \\
\hline 0 & -2.65108 & -0.66822 & 0.20800 \\
\hline $\mathrm{H}$ & -3.47502 & -0.18618 & 0.03620 \\
\hline $\mathrm{H}$ & -2.91886 & -1.57187 & 0.43599 \\
\hline $\mathrm{H}$ & -0.47395 & -3.18266 & 1.91548 \\
\hline $\mathrm{H}$ & 0.80363 & -3.91991 & 0.87985 \\
\hline $\mathrm{H}$ & -0.92307 & -4.35087 & 0.64115 \\
\hline $\mathrm{H}$ & 2.12496 & -2.56968 & -1.25963 \\
\hline $\mathrm{H}$ & 3.34478 & -2.46586 & 0.05624 \\
\hline $\mathrm{H}$ & 3.63217 & -1.63821 & -1.51248 \\
\hline $\mathrm{H}$ & 1.32322 & 3.46641 & -0.33212 \\
\hline $\mathrm{H}$ & 2.88892 & 3.15756 & 0.49151 \\
\hline $\mathrm{H}$ & 1.52552 & 3.90557 & 1.39308 \\
\hline
\end{tabular}




$\begin{array}{lrrr}\mathrm{Cm} & 0.01104 & -0.31086 & -0.03138 \\ \mathrm{O} & -0.49611 & 1.66261 & -1.48992 \\ \mathrm{C} & -0.07355 & 3.06346 & -1.67438 \\ \mathrm{H} & -1.25516 & 1.50121 & -2.06868 \\ \mathrm{O} & -1.99766 & -1.62200 & 0.93023 \\ \mathrm{H} & -2.13409 & -2.58111 & 0.88490 \\ \mathrm{H} & -2.75585 & -1.27162 & 1.42261 \\ \mathrm{O} & 2.30318 & -1.41661 & 0.43104 \\ \mathrm{H} & 3.07195 & -1.46508 & -0.15790 \\ \mathrm{H} & 2.62581 & -1.70420 & 1.29878 \\ \mathrm{O} & -1.42564 & 1.20275 & 1.38666 \\ \mathrm{C} & -2.45041 & 2.25158 & 1.20459 \\ \mathrm{H} & -1.34680 & 1.02751 & 2.33592 \\ \mathrm{O} & 0.35382 & -0.82749 & 2.50320 \\ \mathrm{H} & -0.09999 & -1.51966 & 3.00979 \\ \mathrm{H} & 0.99062 & -0.43175 & 3.11901 \\ \mathrm{O} & 1.47999 & 1.49409 & 0.82981 \\ \mathrm{C} & 2.90613 & 1.87525 & 0.79264 \\ \mathrm{H} & 0.99604 & 2.19942 & 1.28346 \\ \mathrm{O} & -1.77046 & -0.80125 & -1.89193 \\ \mathrm{H} & -2.69677 & -1.04179 & -1.73231 \\ \mathrm{H} & -1.63230 & -0.93207 & -2.84361 \\ \mathrm{O} & 0.10451 & -2.81285 & -0.65553 \\ \mathrm{H} & -0.46207 & -3.33181 & -1.24699 \\ \mathrm{H} & 0.81329 & -3.41303 & -0.37646 \\ \mathrm{O} & 1.35650 & -0.46340 & -2.18575 \\ \mathrm{H} & 1.71803 & -1.24250 & -2.63662 \\ \mathrm{H} & 1.52862 & 0.28497 & -2.77820 \\ \mathrm{H} & 0.89177 & 3.17213 & -1.17720 \\ \mathrm{H} & -0.81462 & 3.73554 & -1.22961 \\ \mathrm{H} & 0.03263 & 3.27049 & -2.74402 \\ \mathrm{H} & -2.61246 & 2.35205 & 0.13010 \\ \mathrm{H} & -2.08909 & 3.19382 & 1.62900 \\ \mathrm{H} & -3.37827 & 1.94384 & 1.69687 \\ \mathrm{H} & 3.40869 & 1.16594 & 0.13255 \\ \mathrm{H} & 3.32511 & 1.82414 & 1.80258 \\ \mathrm{H} & 3.00131 & 2.88931 & 0.39147 \\ & & & \end{array}$

